\title{
MATRIX VALUED SPHERICAL FUNCTIONS ASSOCIATED TO THE THREE DIMENSIONAL HYPERBOLIC SPACE
}

\author{
F. A. GRÜNBAUM \\ Departament of Mathematics, University of California, Berkeley CA 94705 \\ grunbaum@math.berkeley.edu \\ I. PACHARONI* and J. TIRAO ${ }^{\dagger}$ \\ CIEM-FaMAF, Universidad Nacional de Córdoba, Córdoba 5000, Argentina \\ *pacharon@mate.uncor.edu \\ †tirao@mate.uncor.edu
}

Received 8 March 2002

\begin{abstract}
The main purpose of this paper is to compute all irreducible spherical functions on $G=\mathrm{SL}(2, \mathbb{C})$ of arbitrary type $\delta \in \hat{K}$, where $K=\mathrm{SU}(2)$. This is accomplished by associating to a spherical function $\Phi$ on $G$ a matrix valued function $H$ on the three dimensional hyperbolic space $\mathbb{H}=G / K$. The entries of $H$ are solutions of two coupled systems of ordinary differential equations. By an appropriate twisting involving Hahn polynomials we uncouple one of the systems and express the entries of $H$ in terms of Gauss' functions ${ }_{2} F_{1}$. Just as in the compact instance treated in [7], there is a useful role for a special class of generalized hypergeometric functions ${ }_{p+1} F_{p}$.
\end{abstract}

Keywords: Ordinary differential equations; coupled system; generalized hypergeometric functions; matrix valued bispectral property.

Mathematics Subject Classification 2000: 22E20, 33C45

\section{Introduction and Statement of Results}

Let $G$ be a locally compact unimodular group and let $K$ be a compact subgroup of $G$. Let $\hat{K}$ denote the set of all equivalence classes of complex finite dimensional irreducible representations of $K$; for each $\delta \in \hat{K}$, let $\xi_{\delta}$ denote the character of $\delta$, $d(\delta)$ the degree of $\delta$, i.e. the dimension of any representation in the class $\delta$, and $\chi_{\delta}=d(\delta) \xi_{\delta}$. We shall choose once and for all the Haar measure $d k$ on $K$ normalized by $\int_{K} d k=1$.

We shall denote by $V$ a finite dimensional vector space over the field $\mathbb{C}$ of complex numbers and by $\operatorname{End}(V)$ the space of all linear transformations of $V$ into $V$. Whenever we refer to a topology on such a vector space we shall be talking about the unique Hausdorff linear topology on it.

A spherical function $\Phi$ on $G$ of type $\delta \in \hat{K}$ is a continuous function on $G$ with values in $\operatorname{End}(V)$ such that 
(i) $\Phi(e)=I$. ( $I=$ identity transformation $)$.

(ii) $\Phi(x) \Phi(y)=\int_{K} \chi_{\delta}\left(k^{-1}\right) \Phi(x k y) d k$, for all $x, y \in G$.

The reader can find a number of general results in $[6,13]$. For our purpose it is appropriate to recall

Proposition $1.1([6,13])$. If $\Phi: G \longrightarrow \operatorname{End}(V)$ is a spherical function of type $\delta$ then:

(i) $\Phi\left(k g k^{\prime}\right)=\Phi(k) \Phi(g) \Phi\left(k^{\prime}\right)$, for all $k, k^{\prime} \in G, g \in G$.

(ii) $k \mapsto \Phi(k)$ is a representation of $K$ such that any irreducible subrepresentation belongs to $\delta$.

Concerning the definition let us point out that the spherical function $\Phi$ determines its type univocally (Proposition 1.1) and let us say that the number of times that $\delta$ occurs in the representation $k \mapsto \Phi(k)$ is called the height of $\Phi$.

The three dimensional hyperbolic space $\mathbb{H}=\mathbb{C} \times \mathbb{R}^{+}$can be realized as the homogeneous space $G / K$, where $G=\mathrm{SL}(2, \mathbb{C})$ and $K=\mathrm{SU}(2)$. We are interested in determining, up to equivalence, all irreducible spherical functions, associated to the pair $(G, K)$. If $(V, \pi)$ is a finite dimensional irreducible representation of $K$ in the equivalence class $\delta \in \hat{K}$, a spherical function on $G$ of type $\delta$ is characterized, see $[6,13]$, by

(i) $\Phi: G \longrightarrow \operatorname{End}(V)$ is analytic.

(ii) $\Phi\left(k_{1} g k_{2}\right)=\pi\left(k_{1}\right) \Phi(g) \pi\left(k_{2}\right)$, for all $k_{1}, k_{2} \in K, g \in G$, and $\Phi(e)=I$.

(iii) $[\Omega \Phi](g)=\tilde{\lambda} \Phi(g),[\bar{\Omega} \Phi](g)=\tilde{\mu} \Phi(g)$ for all $g \in G$ and for some $\tilde{\lambda}, \tilde{\mu} \in \mathbb{C}$.

Here $\Omega$ and $\bar{\Omega}$ are two algebraically independent generators of the polynomial algebra $D(G)^{G}$ of all differential operators on $G$ which are invariant under left and right multiplication by elements in $G$. A particular choice of these operators is given in Proposition 2.1.

The set $\hat{K}$ can be identified with the set $\mathbb{Z}_{\geq 0}$. If $A \in \mathrm{SU}(2)$ then $\pi(A)=$ $\pi_{\ell}(A)=A^{\ell}$, where $A^{\ell}$ denotes the $\ell$-symmetric power of $A$, defines an irreducible representation of $K$ in the class $\ell \in \mathbb{Z}_{\geq 0}$.

The representation $\pi_{\ell}$ of $K=\mathrm{SU}(2)$ extends to a unique holomorphic representation of $G=\operatorname{SL}(2, \mathbb{C})$, which we shall still denote by $\pi_{\ell}$. Let $\Phi_{\pi}: G \longrightarrow \operatorname{End}\left(V_{\pi}\right)$ be defined by

$$
\Phi_{\pi}(g)=\pi_{\ell}(g)
$$

Then applying the definition it follows that $\Phi_{\pi}$ is a spherical function of type $\ell$. These spherical functions will play a crucial role in the rest of the paper.

We are now in a position to describe the plan of the paper. Section 2 contains a brief review of standard facts. 
To determine all spherical functions $\Phi: G \longrightarrow \operatorname{End}\left(V_{\pi}\right)$ of type $\pi=\pi_{\ell}$, we use the function $\Phi_{\pi}$ to define a function $H: G \longrightarrow \operatorname{End}\left(V_{\pi}\right)$ by

$$
H(g)=\Phi(g) \Phi_{\pi}(g)^{-1} .
$$

Then $H$ satisfies

(i) $H(e)=I$.

(ii) $H(g k)=H(g)$, for all $g \in G, k \in K$.

(iii) $H(k g)=\pi(k) H(g) \pi\left(k^{-1}\right)$, for all $g \in G, k \in K$.

Property (ii) says that $H$ may be considered as a function on the hyperbolic space $\mathbb{H}$.

The fact that $\Phi$ is an eigenfunction of $\Omega$ and $\bar{\Omega}$, makes $H$ into an eigenfunction of certain differential operators $D$ and $E$ on $\mathbb{H}$. This is done in Sec. 3. For completeness the explicit computation of these operators is carried out fully in the Appendix.

In Sec. 4, we take full advantage of the $K$-orbit structure of $\mathbb{H}$ combined with property (iii) of our functions $H$. The $K$-orbits in $\mathbb{H}$ are the spheres with center in the positive axis $\{(0, r): r>0\}$ with north and south poles of the form $(0, s)$ and $\left(0, \frac{1}{s}\right)$, and the single point set $\{(0,1)\}$. Thus the set of $K$-orbits in $\mathbb{H}$ is parametrized by the interval $(0,1]$ or by $[1, \infty)$.

It follows that there exist ordinary differential operators $\tilde{D}$ and $\tilde{E}$ acting on the space $C^{\infty}((0,1)) \otimes \operatorname{End}\left(V_{\pi}\right)$ such that

$$
(D H)(0, r)=(\tilde{D} \tilde{H})(r), \quad(E H)(0, r)=(\tilde{E} \tilde{H})(r),
$$

where $\tilde{H}(r)=H(0, r), r \in(0,1)$. These operators $\tilde{D}$ and $\tilde{E}$ are explicitly given in Theorems 4.2 and 4.3. We need to compute a number of second order partial derivatives of the function $H: \mathbb{H} \longrightarrow \operatorname{End}\left(V_{\pi}\right)$ at the point $(0, r)$. This detailed computation is broken down in a number of lemmas included in the Appendix for the benefit of the reader.

Theorems 4.2 and 4.3 are given in terms of linear transformations. The functions $\tilde{H}$ turn out to be diagonalizable (Lemma 4.4). Thus, in an appropriate basis of $V_{\pi}$ we can write $\tilde{H}(r)=\left(\tilde{h}_{0}(r), \ldots, \tilde{h}_{\ell}(r)\right)$. Then we give in Corollaries 4.6 and 4.7 the corresponding statements of these theorems in terms of the scalar functions $\tilde{h}_{i}$.

We also introduce here the variable $t=r^{2}$ which converts the differential operators $\tilde{D}$ and $\tilde{E}$ into new operators $D$ and $E$, and the functions $\tilde{H}, \tilde{h}_{i}$ into the functions $H, h_{i}$ defined by $H(t)=\tilde{H}(\sqrt{t})$ and $h_{i}(t)=\tilde{h}_{i}(\sqrt{t})$. At this point there is a slight abuse of notation. The resulting equation $D H=\lambda H$ is a coupled system of $\ell+1$ second order differential equations in the components $\left(h_{0}, \ldots, h_{\ell}\right)$ of $H$. Fortunately the coupling matrix $C_{0}+C_{1}$ of the system $D H=\lambda H$ is symmetric, with eigenvalues $-j(j+1), 0 \leq j \leq \ell$, and eigenvectors $u_{j}$ whose components $u_{i, j}$ are given by certain Hahn orthogonal polynomials, see Proposition 5.1. Let $U$ denote the $(\ell+1) \times(\ell+1)$ matrix $\left(u_{i, j}\right)$ which will play a crucial role. In fact, this $U$ allows us to decouple the system above. More is true, the twisting $\check{H}=U^{-1} H$ leads us to Gauss' hypergeometric equation, a fact that we exploit fully. 
By well known reasons the smooth functions $H:(0,1) \longrightarrow \mathbb{C}^{\ell+1}$ that satisfy $D H=\lambda H$ are analytic functions and the dimension of the corresponding eigenspace $V_{\lambda}$ is $2(\ell+1)$.

To get a handle on $V_{\lambda}$ we consider functions $H \in V_{\lambda}$ of the form $H(t)=t^{p} F(t)$, $p \in \mathbb{C}$, with $F$ analytic at $t=0$ and $F(0) \neq 0$. This forces $\lambda=4 p(p-1)$. Observe that $p$ and $1-p$ give rise to the same $\lambda$. Indeed any statement valid for $\operatorname{Re}(2 p) \geq 1$ has a mirror image for $\operatorname{Re}(2 p) \leq 1$ obtained by changing $p$ into $1-p$.

Let $V(p)$ be the linear subspace of $V_{\lambda}, \lambda=4 p(p-1)$, of all $H \in V_{\lambda}$ of the form $H(t)=t^{p} F(t)$ with $F$ analytic at $t=0$.

When $2 p$ is not an integer we prove that $V_{\lambda}=V(p) \oplus V(1-p)$. When $2 p$ is an integer the situation is more complicated: if $2 p \geq 1$ we have $V(p) \subset V(1-p)$ and $\operatorname{dim} V(p)=\ell+1, \operatorname{dim} V(1-p)=\min \{2(\ell+1), \ell+2 p\}$. The situation when $2 p \leq 0$ can be read off by exchanging $p$ by $1-p$, see Proposition 5.7.

When $2 p \notin \mathbb{Z}$ we consider the linear map $\eta: V(p) \longrightarrow \mathbb{C}^{\ell+1}$ defined by $\eta(H)=$ $\lim _{t \rightarrow 0^{+}}\left(t^{-p} H(t)\right)$. It turns out that $\eta$ is an isomorphism and by using it $E$ is described by an $(\ell+1) \times(\ell+1)$ matrix $L(p)$, see Proposition 6.2. The eigenvalues of $L(p)$ are the same as those of $L(1-p)$, all of them with algebraic multiplicity one. Using Proposition 6.7 combining the eigenvectors of $L(p)$ and $L(1-p)$ with the same eigenvalue $\mu$ we obtain all simultaneous solutions of the system $D H=\lambda H$ and $E H=\mu H$. To get our hands on the spherical functions we need to focus on those $H$ 's arising from the linear combination of eigenvectors of $L(p)$ and $L(1-p)$ with the same eigenvalue which insures that $\lim _{t \rightarrow 1^{-}} H(t)=(1, \ldots, 1)$.

This requirement leads us to consider the linear subspace $W_{\lambda}$ of $V_{\lambda}$ consisting of all $H$ such that $\lim _{t \rightarrow 1^{-}} H(t)$ is finite. For any $p \in \mathbb{C}$ with $\operatorname{dim} V(p)=\ell+1, W_{\lambda}$ turns out to be a useful direct complement of $V(p)$ in $V_{\lambda}$, see Propositions 5.9 and 5.10. Furthermore $E$ preserves $W_{\lambda}$ and the linear isomorphism $\eta: W_{\lambda} \longrightarrow \mathbb{C}^{\ell+1}$ defined by

$$
\eta(H)= \begin{cases}\lim _{t \rightarrow 0^{+}} t^{p-1} H(t) & \text { if } \operatorname{Re}(2 p)>1, \\ \lim _{t \rightarrow 0^{+}} t^{-p} H(t) & \text { if } \operatorname{Re}(2 p)<1, \\ \lim _{t \rightarrow 0^{+}} t^{p-1} P(H)(t) & \text { if } \operatorname{Re}(2 p)=1 \text { and } 2 p \neq 1, \\ \lim _{t \rightarrow 0^{+}}\left(t^{\frac{1}{2}} \log t\right)^{-1} H(t) & \text { if } 2 p=1,\end{cases}
$$

reduces the determination of all spherical functions to a linear algebra problem. More precisely, since we establish in Propositions 6.4, 6.5 and 6.6, that, in $W_{\lambda}$, $E=\eta^{-1} L(1-p) \eta$ it suffices to find the eigenvectors of $L(1-p)$. See Proposition 6.6 for the definition of the projection $P$ appearing in (1.1).

Proposition 6.7 determines the eigen-structure of $L(p)$ and Theorem 6.8 gives the main result of the paper, namely an explicit expression for all spherical functions. The section closes with a look at the relation between our bare hands construction and the generalized (also known as nonunitary) principal series representations of $\mathrm{SL}(2, \mathbb{C})$. 
Section 7 displays explicit expressions of all spherical functions for low values of $\ell$. The reader might use these examples to get a better feeling for the results in the paper. For instance we use these explicit expressions to see that as a function of $p$, $H(t, p)$ is a meromorphic function with a finite number of removable singularities. The values at these singularities are exactly the cases when the expression for $H(t, p)$ involves the term $\log t$. We also formulate a conjecture expressing our spherical functions in terms of generalized hypergeometric functions.

In Sec. 8, we discuss some generalities derived from the definition of unitary spherical functions and we pick among all spherical functions of our pair $(G, K)$ those that are unitarizable, see Corollary 8.11.

In Sec. 9, we observe that a matrix valued function $\Phi(t, p)$ put together from the functions $H_{0}(t, p), \ldots, H_{\ell}(t, p)$ alluded to in the comments preceding Theorem 6.8 satisfies not only differential equations in $t$ but also a difference equation in $p$.

Finally in the Appendix we collect a number of explicit computations for the benefit of the reader.

There is a point of contact between the present paper and [14]. By considering spherical functions associated to generalized principal series representations A. Wang derives two coupled systems of differential equations closely related to the systems in Corollary 4.8. From these systems, the author obtains a single second order differential equation for the last component, and points out that, in principle, this gives a way of finding the remaining components.

Finally we remark that we have chosen this example as one of the simplest to analyze among the noncompact symmetric spaces of rank one leading to matrix valued spherical functions. We hope to deal with other simple examples in the near future. The consideration of some more general examples appears, at this point, to be a very interesting challenge.

\section{Preliminaries}

We first review well known facts about the structure of the real Lie algebra $\mathfrak{s l}(2, \mathbb{C})$ and of the center of its universal enveloping algebra. We also collect here some useful facts about the quotient $\mathrm{SL}(2, \mathbb{C}) / \mathrm{SU}(2)$. A basis of $\mathfrak{g}=\mathfrak{s l}(2, \mathbb{C})$ over $\mathbb{R}$ is given by

$$
\begin{aligned}
& H_{1}=\left[\begin{array}{cc}
1 & 0 \\
0 & -1
\end{array}\right], \quad W_{1}=\left[\begin{array}{cc}
0 & 1 \\
-1 & 0
\end{array}\right], \quad V_{1}=\left[\begin{array}{ll}
0 & 1 \\
1 & 0
\end{array}\right], \\
& H_{2}=\left[\begin{array}{cc}
i & 0 \\
0 & -i
\end{array}\right], \quad W_{2}=\left[\begin{array}{ll}
0 & i \\
i & 0
\end{array}\right], \quad V_{2}=\left[\begin{array}{cc}
0 & i \\
-i & 0
\end{array}\right] .
\end{aligned}
$$

We note that $H_{1}, W_{1}$ and $V_{1}$ generate a Lie subalgebra isomorphic to $\mathfrak{s l}(2, \mathbb{R})$, and that $H_{2}, W_{1}$ and $W_{2}$ form a basis of the Lie algebra of $K$.

The following are elements in the complexification $\mathfrak{g}_{\mathbb{C}}$ of the Lie algebra $\mathfrak{g}$. 


$$
\begin{aligned}
& T=\frac{1}{2}\left(H_{1}-i H_{2}\right), \quad V=\frac{1}{2}\left(V_{1}-i W_{2}\right), \quad W=\frac{1}{2}\left(W_{1}-i V_{2}\right) . \\
& \bar{T}=\frac{1}{2}\left(H_{1}+i H_{2}\right), \quad \bar{V}=\frac{1}{2}\left(V_{1}+i W_{2}\right), \quad \bar{W}=\frac{1}{2}\left(W_{1}+i V_{2}\right) .
\end{aligned}
$$

Proposition 2.1. $D(G)^{G}$ as a polynomial algebra is generated by the algebraically independent elements

$$
\Omega=T^{2}+V^{2}-W^{2} \text { and } \bar{\Omega}=\bar{T}^{2}+\bar{V}^{2}-\bar{W}^{2} .
$$

In the real basis of $\mathfrak{s l}(2, \mathbb{C})$ we have

$$
\begin{aligned}
& 4 \Omega=H_{1}^{2}+V_{1}^{2}+V_{2}^{2}-\left(H_{2}^{2}+W_{1}^{2}+W_{2}^{2}\right)-2 i\left(H_{1} H_{2}+V_{1} W_{2}-V_{2} W_{1}\right), \\
& 4 \bar{\Omega}=H_{1}^{2}+V_{1}^{2}+V_{2}^{2}-\left(H_{2}^{2}+W_{1}^{2}+W_{2}^{2}\right)+2 i\left(H_{1} H_{2}+V_{1} W_{2}-V_{2} W_{1}\right) .
\end{aligned}
$$

We have a function from $G=\operatorname{SL}(2, \mathbb{C})$ into the space of all $2 \times 2$ symmetric positive definite matrices, given by $g \mapsto g g^{*}$. This function factors through the quotient $G / K$, because $k k^{*}=I$. Explicitly we have,

$$
\left(\begin{array}{ll}
a & b \\
c & d
\end{array}\right) \longmapsto\left(\begin{array}{cc}
|a|^{2}+|b|^{2} & a \bar{c}+b \bar{d} \\
\bar{a} c+\bar{b} d & |c|^{2}+|d|^{2}
\end{array}\right) .
$$

If we put

$$
r=|c|^{2}+|d|^{2} \quad \text { and } z=a \bar{c}+b \bar{d},
$$

we have $|a|^{2}+|b|^{2}=\frac{1+|z|^{2}}{r}$ (since $g g^{*}$ has determinant one) and

$$
g g^{*}=\left(\begin{array}{cc}
\frac{1+|z|^{2}}{r} & z \\
\bar{z} & r
\end{array}\right)
$$

Then we can identify the quotient $G / K$ with

$$
\mathbb{H}=\left\{(z, r): z \in \mathbb{C}, r \in \mathbb{R}_{>0}\right\} .
$$

The projection map $p: G \longrightarrow \mathbb{H}$ is given by $p(g)=\left(g g^{*}\right)\left(\begin{array}{l}0 \\ 1\end{array}\right)=(z, r)$. To simplify notation we identify row and column vectors.

The left multiplication on the group $G$ induces the action of $G$ in $\mathbb{H}$ given by

$$
A(z, r)=A\left(\begin{array}{cc}
\frac{1+|z|^{2}}{r} & z \\
\bar{z} & r
\end{array}\right) A^{*}\left(\begin{array}{l}
0 \\
1
\end{array}\right)=\left(z^{*}, r^{*}\right)
$$

More explicitly if $g=\left(\begin{array}{ll}a & b \\ c & d\end{array}\right)$ and $p=(z, r)$ we have $g \cdot p=\left(z^{*}, r^{*}\right)$ with

$$
z^{*}=\frac{a \bar{c}+(a z+b r)(\bar{c} \bar{z}+\bar{d} r)}{r} \quad \text { and } r^{*}=\frac{|c|^{2}+|c z+d r|^{2}}{r}
$$




\section{Reduction to $G / K$}

Any irreducible finite dimensional representation of $K=\mathrm{SU}(2)$ is of the form $\pi_{\ell}(k)=k^{\ell}$, where $k^{\ell}$ denotes the $\ell$-symmetric power of $k$. The representation $\pi_{\ell}$ extends to a unique holomorphic representation of $\mathrm{SL}(2, \mathbb{C})$ and we also denote it by $\pi_{\ell}$. In either case, we denote by $\dot{\pi}=\dot{\pi}_{\ell}$ the corresponding derivative of $\pi$ at the identity. Note that $\dot{\pi}: \mathfrak{g} \longrightarrow \operatorname{End}\left(V_{\pi}\right)$ is $\mathbb{C}$-linear.

We define for each representation $\pi=\pi_{\ell}$ of $K$ a function $\Phi_{\pi}: G \longrightarrow \operatorname{End}\left(V_{\pi}\right)$ given by

$$
\Phi_{\pi}(g)=\pi(g) .
$$

In order to determine all spherical functions $\Phi: G \longrightarrow \operatorname{End}(V)$ we find it useful to introduce the function $H$ given by

$$
H(g)=\Phi(g) \Phi_{\pi}(g)^{-1} .
$$

This function $H$ satisfies

(i) $H(e)=I$.

(ii) $H(g k)=H(g)$, for $g \in G, k \in K$.

(iii) $H(k g)=\pi(k) H(g) \pi\left(k^{-1}\right)$, for $g \in G, k \in K$.

Property (ii) says that $H$ can be considered as a function on the quotient $G / K$. The fact that $\Phi$ is an eigenfunction of $\Omega$ and $\bar{\Omega}$ makes the function $H$, introduced before, into an eigenfunction of certain differential operators on $\mathbb{H}$, to be determined now.

Let

$$
\begin{aligned}
D H= & \left(H_{1}^{2}+V_{1}^{2}+V_{2}^{2}\right)(H) \\
E H= & H_{1}(H) \Phi_{\pi} \dot{\pi}\left(H_{1}\right) \Phi_{\pi}^{-1}+V_{1}(H) \Phi_{\pi} \dot{\pi}\left(V_{1}\right) \Phi_{\pi}^{-1} \\
& +V_{2}(H) \Phi_{\pi} \dot{\pi}\left(V_{2}\right) \Phi_{\pi}^{-1} .
\end{aligned}
$$

Proposition 3.1. For any $H \in C^{\infty}(G) \otimes \operatorname{End}\left(V_{\pi}\right)$ right invariant under $K$, the function $\Phi=H \Phi_{\pi}$ satisfies $(4 \bar{\Omega}) \Phi=\tilde{\lambda} \Phi$ and $(\Omega-\bar{\Omega}) \Phi=\tilde{\mu} \Phi$ if and only if $H$ satisfies $D H=\lambda H$ and $E H=\mu H$, with

$$
\tilde{\lambda}=\lambda \quad \text { and } \tilde{\mu}=\mu+\ell(\ell+2),
$$

when $\pi=\pi_{\ell}$.

Proof. If $X \in \mathfrak{k}$ then $X(H)=0$. In particular

$$
H_{2}(H)=W_{1}(H)=W_{2}(H)=0 .
$$

Therefore $T(H)=\bar{T}(H)=\frac{1}{2} H_{1}(H), V(H)=\bar{V}(H)=\frac{1}{2} V_{1}(H), W(H)=$ $-\bar{W}(H)=-\frac{i}{2} V_{2}(H)$, and

$$
4 \Omega(H)=4 \bar{\Omega}(H)=\left(H_{1}^{2}+V_{1}^{2}+V_{2}^{2}\right)(H)=D H .
$$


We also have

$$
\begin{aligned}
\Omega\left(H \Phi_{\pi}\right)= & \Omega(H) \Phi_{\pi}+H \Omega\left(\Phi_{\pi}\right)+2 T(H) T\left(\Phi_{\pi}\right) \\
& +2 V(H) V\left(\Phi_{\pi}\right)-2 W(H) W\left(\Phi_{\pi}\right) \\
= & \Omega(H) \Phi_{\pi}+H \Phi_{\pi} \dot{\pi}(\Omega)+H_{1}(H) \Phi_{\pi} \dot{\pi}(T) \\
& +V_{1}(H) \Phi_{\pi} \dot{\pi}(V)+i V_{2}(H) \Phi_{\pi} \dot{\pi}(W)
\end{aligned}
$$

and

$$
\begin{aligned}
\bar{\Omega}\left(H \Phi_{\pi}\right)= & \bar{\Omega}(H) \Phi_{\pi}+H \Phi_{\pi} \dot{\pi}(\bar{\Omega})+H_{1}(H) \Phi_{\pi} \dot{\pi}(\bar{T}) \\
& +V_{1}(H) \Phi_{\pi} \dot{\pi}(\bar{V})-i V_{2}(H) \Phi_{\pi} \dot{\pi}(\bar{W})
\end{aligned}
$$

Note that if $Y=i X$, for $X \in \mathfrak{g}$ then $\dot{\pi}(Y)=i \dot{\pi}(X)$. In particular we have $\dot{\pi}(\bar{T})=\dot{\pi}(\bar{V})=\dot{\pi}(\bar{W})=0$. Therefore

$$
\begin{aligned}
4 \bar{\Omega}\left(H \Phi_{\pi}\right) & =(D H) \Phi_{\pi}+4 H \Phi_{\pi} \dot{\pi}(\bar{\Omega}), \\
(\Omega-\bar{\Omega})\left(H \Phi_{\pi}\right) & =H \Phi_{\pi} \dot{\pi}(\Omega-\bar{\Omega})+E H \Phi_{\pi} .
\end{aligned}
$$

Finally the representation theory of $\operatorname{SL}(2, \mathbb{C})$ gives, for $\pi=\pi_{\ell}, \dot{\pi}(\bar{\Omega})=0$ and $\dot{\pi}(\Omega)=\ell(\ell+2) I$. Now the proposition follows easily.

Given $H \in C^{\infty}(\mathbb{H}) \otimes \operatorname{End}\left(V_{\pi}\right)$ we shall also denote by $H \in C^{\infty}(G) \otimes \operatorname{End}\left(V_{\pi}\right)$ the function defined by $H(g)=H(p(g)), g \in G$. Moreover, if $F$ is a linear endomorphism of $C^{\infty}(G) \otimes \operatorname{End}\left(V_{\pi}\right)$ which preserves the subspace $C^{\infty}(G)^{K} \otimes \operatorname{End}\left(V_{\pi}\right)$ of all functions which are right invariant by elements in $K$, then we shall also denote by $F$ the endomorphism of $C^{\infty}(\mathbb{H}) \otimes \operatorname{End}\left(V_{\pi}\right)$ which satisfies $F(H)(p(g))=F(H)(g)$, $g \in G, H \in C^{\infty}(\mathbb{H}) \otimes \operatorname{End}\left(V_{\pi}\right)$.

Lemma 3.2. The differential operators $D$ and $E$ introduced in (3.1) and (3.2), define differential operators $D$ and $E$ acting on $C^{\infty}(\mathbb{H}) \otimes \operatorname{End}\left(V_{\pi}\right)$.

Proof. The only thing we really need to prove is that $D$ and $E$ preserve the subspace $C^{\infty}(G)^{K} \otimes \operatorname{End}\left(V_{\pi}\right)$.

It is easy to see that $D=H_{1}^{2}+V_{1}^{2}+V_{2}^{2}=-\left(H_{2}^{2}+W_{1}^{2}+W_{2}^{2}\right)$ is a multiple of the Casimir operator of $K$. Then $D$ preserves $C^{\infty}(G)^{K} \otimes \operatorname{End}\left(V_{\pi}\right)$.

Let us now check that $E$ has the same property. Since $K$ is connected this is equivalent to verifying that for any $X \in \mathfrak{k}$ and all $H \in C^{\infty}(G)^{K} \otimes \operatorname{End}\left(V_{\pi}\right)$ we have $X(E H)=0$, which in turns amounts to prove that

$$
\begin{aligned}
& X H_{1}(H) \Phi_{\pi} \dot{\pi}\left(H_{1}\right)+H_{1}(H) X\left(\Phi_{\pi}\right) \dot{\pi}\left(H_{1}\right)-H_{1}(H) \Phi_{\pi} \dot{\pi}\left(H_{1}\right) \dot{\pi}(X) \\
& \quad+X V_{1}(H) \Phi_{\pi} \dot{\pi}\left(V_{1}\right)+V_{1}(H) X\left(\Phi_{\pi}\right) \dot{\pi}\left(V_{1}\right)-V_{1}(H) \Phi_{\pi} \dot{\pi}\left(V_{1}\right) \dot{\pi}(X) \\
& \quad+X V_{2}(H) \Phi_{\pi} \dot{\pi}\left(V_{2}\right)+V_{2}(H) X\left(\Phi_{\pi}\right) \dot{\pi}\left(V_{2}\right)-V_{2}(H) \Phi_{\pi} \dot{\pi}\left(V_{2}\right) \dot{\pi}(X)=0 .
\end{aligned}
$$


Because $X(H)=0$ and $X\left(\Phi_{\pi}\right)=\Phi_{\pi} \dot{\pi}(X)$, this is also equivalent to showing that

$$
\begin{aligned}
& {\left[X, H_{1}\right](H) \Phi_{\pi} \dot{\pi}\left(H_{1}\right)+H_{1}(H) \Phi_{\pi} \dot{\pi}\left(\left[X, H_{1}\right]\right)+\left[X, V_{1}\right](H) \Phi_{\pi} \dot{\pi}\left(V_{1}\right)} \\
& \quad+V_{1}(H) \Phi_{\pi} \dot{\pi}\left(\left[X, V_{1}\right]\right)+\left[X, V_{2}\right](H) \Phi_{\pi} \dot{\pi}\left(V_{2}\right)+V_{2}(H) \Phi_{\pi} \dot{\pi}\left(\left[X, V_{2}\right]\right)=0 .
\end{aligned}
$$

If we put $X=H_{1}$ in (3.3) and use $\left[H_{1}, V_{1}\right]=2 W_{1},\left[H_{1}, V_{2}\right]=2 W_{2}, W_{2}=i V_{1}$, $V_{2}=i W_{1}$ we get

$$
\begin{aligned}
& 2 W_{1}(H) \Phi_{\pi} \dot{\pi}\left(V_{1}\right)+2 V_{1}(H) \Phi_{\pi} \dot{\pi}\left(W_{1}\right) \\
& \quad+2 W_{2}(H) \Phi_{\pi} \dot{\pi}\left(V_{2}\right)+2 V_{2}(H) \Phi_{\pi} \dot{\pi}\left(W_{2}\right)=0 .
\end{aligned}
$$

If we substitute $X=\frac{1}{2}\left(V_{1}+W_{1}\right)$ in $(3.3)$ and use $\left[H_{1}, X\right]=2 X,\left[X, V_{1}\right]=H_{1}$, $\left[X, V_{2}\right]=-i H_{1}$, we obtain

$$
\begin{aligned}
& -V_{1}(H) \Phi_{\pi} \dot{\pi}\left(H_{1}\right)-W_{1}(H) \Phi_{\pi} \dot{\pi}\left(H_{1}\right) \\
& \quad-H_{1}(H) \Phi_{\pi} \dot{\pi}\left(V_{1}\right)-H_{1}(H) \Phi_{\pi} \dot{\pi}\left(W_{1}\right)+H_{1}(H) \Phi_{\pi} \dot{\pi}\left(V_{1}\right)+V_{1}(H) \Phi_{\pi} \dot{\pi}\left(H_{1}\right) \\
& \quad-i H_{1}(H) \Phi_{\pi} \dot{\pi}\left(V_{2}\right)-i V_{2}(H) \Phi_{\pi} \dot{\pi}\left(H_{1}\right)=0,
\end{aligned}
$$

since $V_{2}=i W_{1}, W_{2}=i V_{1}$. Now the representation theory of $\operatorname{SL}(2, \mathbb{C})$ tell us that $X(E H)=0$ for all $X \in \mathfrak{k}$. This finishes the proof of the lemma.

Now we give the expressions of the operators $D$ and $E$ on $\mathbb{H}$ in the real linear coordinates $(x, y, r)$ defined by $z=x+i y$. The detailed proofs appear in the Appendix at the end of the paper.

Proposition 3.3. For any $H \in C^{\infty}(\mathbb{H}) \otimes \operatorname{End}\left(V_{\pi}\right)$ we have

$$
\begin{aligned}
\frac{1}{4} D H= & \left(\operatorname{Re}^{2} z+1\right) H_{x x}+\left(\operatorname{Im}^{2} z+1\right) H_{y y}+r^{2} H_{r r}+2 \operatorname{Re} z \operatorname{Im} z H_{x y} \\
& +2 r \operatorname{Re} z H_{x r}+2 r \operatorname{Im} z H_{y r}+3 \operatorname{Re} z H_{x}+3 \operatorname{Im} z H_{y}+3 r H_{r} .
\end{aligned}
$$

Proposition 3.4. For any $H \in C^{\infty}(\mathbb{H}) \otimes \operatorname{End}\left(V_{\pi}\right)$ we have

$$
\begin{aligned}
E H= & H_{x} \dot{\pi}\left(\begin{array}{cc}
z-\bar{z} & \frac{2\left(1+|z|^{2}\right)}{r} \\
2 r & \bar{z}-z
\end{array}\right) \\
& +H_{y} \dot{\pi}\left(\begin{array}{cc}
i(z+\bar{z}) & \frac{2 i\left(1+|z|^{2}\right)}{r} \\
-2 i r & i(z+\bar{z})
\end{array}\right) \\
& +H_{r} \dot{\pi}\left(\begin{array}{cc}
-2 r & 4 z \\
0 & 2 r
\end{array}\right) .
\end{aligned}
$$

Summarizing the results of this section: to determine a simultaneous $C^{\infty}$ eigenfunction $\Phi$ of $\Omega$ and $\bar{\Omega}$ on $G$ satisfying $\Phi\left(k_{1} g k_{2}\right)=\pi\left(k_{1}\right) \Phi(g) \pi\left(k_{2}\right)$ for all $k_{1}, k_{2} \in K, g \in G$, is equivalent to finding a $C^{\infty}$-eigenfunction $H=\Phi \Phi_{\pi}^{-1}$ of $D$ 
and $E$ on $\mathbb{H}$ satisfying $H(k \cdot p)=\pi(k) H(p) \pi\left(k^{-1}\right)$ for all $k \in K, p \in \mathbb{H}$. The relation among the eigenvalues is given in Proposition 3.1.

\section{Reduction to One Variable}

We are interested in considering the differential operators $D$ and $E$ acting on functions $H \in C^{\infty}(\mathbb{H}) \otimes \operatorname{End}\left(V_{\pi}\right)$ such that

$$
H(k \cdot p)=\pi(k) H(p) \pi(k)^{-1}, \quad \text { for all } k \in K \text { and } p \in \mathbb{H} .
$$

This property of $H$ allows us to find ordinary differential operators $\tilde{D}$ and $\tilde{E}$ acting on functions defined on the interval $(0,1)$ such that

$$
(D H)(0, r)=(\tilde{D} \tilde{H})(r), \quad(E H)(0, r)=(\tilde{E} \tilde{H})(r),
$$

where $\tilde{H}(r)=H(0, r)$.

We need to know the $K$-orbit structure of $\mathbb{H}$ : the orbits of $K$ in $\mathbb{H}$ are the spheres with center in the positive axis $\{(0, r): r>0\}$ with north and south poles of the form $(0, s)$ and $\left(0, s^{-1}\right)$, and the single point set $\{(0,1)\}$. The point $(0,1)$ will be denoted from now on by $o=(0,1)$. Thus the set of $K$-orbits in $\mathbb{H}$ is parametrized by the interval $(0,1]$ or by $[1, \infty)$.

We introduce now two important characters in the story, $\mathbb{H}^{\times}$and $M$. Let $\mathbb{H}^{\times}=$ $\mathbb{H}-\{o\}$ and put $M=\{k \in K: k \cdot(0, r)=(0, r)\}, 0<r<1$. For any such $r$ we get

$$
M=\left\{\left(\begin{array}{cc}
e^{i \theta} & 0 \\
0 & e^{-i \theta}
\end{array}\right): \theta \in \mathbb{R}\right\} .
$$

Notice that $M$ coincides with the centralizer in $K$ of the Abelian subgroup $A$ of $G$ given by

$$
A=\left\{\left(\begin{array}{cc}
s & 0 \\
0 & s^{-1}
\end{array}\right): s>0\right\} .
$$

Let $\psi: K / M \times(0,1) \longrightarrow \mathbb{H}^{\times}$be defined by

$$
\psi(k M, s)=k \cdot(0, s) .
$$

From the orbit structure discussed above it follows that $\psi$ is a $C^{\infty}$-bijection. To give the expression of the operators $\tilde{D}$ and $\tilde{E}$, obtained by restriction of $D$ and $E$ given in Propositions 3.3 and 3.4, we need to compute a number of first and second order partial derivatives of the function $H$ at the point $(0, r)$.

To begin with we note the following immediate results

$$
H_{r}(0, r)=\frac{d \tilde{H}}{d r}(r) \quad \text { and } \quad H_{r r}(0, r)=\frac{d^{2} \tilde{H}}{d r^{2}}(r) .
$$

To compute the other partial derivatives of the function $H$ in $\mathbb{H}^{\times}$we need to define locally a pair of $C^{\infty}$-functions on $\mathbb{H}^{\times}, k=k(z, r)$ and $s=s(z, r)$ with values in $K$ and in the open interval $(0,1)$, respectively, such that $k \cdot(z, r)=(0, s)$. 
In the open set $\mathbb{H}-\{(0, r): r \geq 1\}$ these functions will be explicitly given below. Similarly one can define a pair of functions $k, s$ in the open set $\mathbb{H}-\{(0, r): 0<$ $r \leq 1\}$

None of these functions can be extended to all of $\mathbb{H}^{\times}$in a continuous fashion. Moreover there is no such a pair of continuous globally defined functions $k, s$. This would imply the existence of a continuous section of $K / M$, which would make $K$ homeomorphic to $K / M \times M$. Now $K$ is simply connected and $M$ is not.

If $(z, r) \in \mathbb{H}-\{(0, r): r \geq 1\}$ we look for $k=\left(\begin{array}{cc}a & b \\ -\bar{b} & \bar{a}\end{array}\right) \in \mathrm{SU}(2)$ such that $k \cdot(z, r)=(0, s), 0<s<1$. This holds if and only if

$$
k\left(\begin{array}{cc}
\left(1+|z|^{2}\right) / r & z \\
\bar{z} & r
\end{array}\right) k^{*}=\left(\begin{array}{cc}
s^{-1} & 0 \\
0 & s
\end{array}\right) .
$$

Then $s$ and $s^{-1}$ are the eigenvalues of $\left(\begin{array}{cc}\frac{1+|z|^{2}}{\frac{r}{z}} & z \\ r\end{array}\right)$. The characteristic equation is

$$
r x^{2}-x\left(1+|z|^{2}+r^{2}\right)+r=0,
$$

thus

$$
\left\{s, s^{-1}\right\}=\left\{\frac{1}{2 r}\left(1+|z|^{2}+r^{2} \pm \sqrt{\left(|z|^{2}+(r+1)^{2}\right)\left(|z|^{2}+(r-1)^{2}\right)}\right)\right\} .
$$

Therefore the eigenvalue that satisfies $0<s<1$ is

$$
s=s(z, r)=\frac{1}{2 r}\left(1+|z|^{2}+r^{2}-\sqrt{\left(|z|^{2}+(r+1)^{2}\right)\left(|z|^{2}+(1-r)^{2}\right)}\right) .
$$

We have thus defined the function $s$. Now we can take as a function $k=k(z, r)$ the one given by taking $k=\left(\begin{array}{cc}a & b \\ -\bar{b} & \bar{a}\end{array}\right) \in \mathrm{SU}(2)$ with

$$
a=\frac{1-r s}{\sqrt{(1-r s)^{2}+(s|z|)^{2}}} \quad \text { and } \quad b=\frac{s z}{\sqrt{(1-r s)^{2}+(s|z|)^{2}}} .
$$

The explicit expressions above for $s$ and $k$ give as a pair of $C^{\infty}$-functions with the desired properties in the appropriate domain.

The inverse of the $C^{\infty}$-bijection $\psi$, defined in (4.1), in $\mathbb{H}-\{(0, r): r \geq 1\}$ is given by

$$
\psi^{-1}(z, r)=\left(k(z, r)^{-1} M, s(z, r)\right)
$$

making clearly that $\psi: K / M \times(0,1) \longrightarrow \mathbb{H}^{\times}$is a diffeomorphism.

Returning now to the main goal of this section, let $\left(C^{\infty}\left(\mathbb{H}^{\times}\right) \otimes \operatorname{End}\left(V_{\pi}\right)\right)^{K}$ be the space of all $C^{\infty}$-functions $H$ on $\mathbb{H}^{\times}$with values in $\operatorname{End}\left(V_{\pi}\right)$ such that

$$
H(k \cdot p)=\pi(k) H(p) \pi\left(k^{-1}\right) \quad \text { for all } k \in K, p \in \mathbb{H}^{\times} .
$$

We will also need to consider the space $C^{\infty}((0,1)) \otimes \operatorname{End}_{M}\left(V_{\pi}\right)$ of all $C^{\infty}$-functions $\tilde{H}$ on the open interval $(0,1)$ with values in $\operatorname{End}\left(V_{\pi}\right)$ with the extra requirement that

$$
\tilde{H}(s)=\pi(m) \tilde{H}(s) \pi\left(m^{-1}\right) \quad \text { for all } m \in M, 0<s<1 \text {. }
$$


Now it is clear that the restriction $H \longrightarrow \tilde{H}$ defines an injective linear map from $\left(C^{\infty}\left(\mathbb{H}^{\times}\right) \otimes \operatorname{End}\left(V_{\pi}\right)\right)^{K}$ into $C^{\infty}((0,1)) \otimes \operatorname{End}_{M}\left(V_{\pi}\right)$. Moreover this map is a surjective isomorphism as follows from the following construction. Given $\tilde{H} \in$ $C^{\infty}((0,1)) \otimes \operatorname{End}_{M}\left(V_{\pi}\right)$ define $H(z, r)=\pi(k) \tilde{H}(s) \pi\left(k^{-1}\right)$ if $(z, r)=\psi(k M, s)$.

The differential operators $D$, and $E$, being invariant under the action of $K$ in $\mathbb{H}^{\times}$, leave stable the subspace $\left(C^{\infty}\left(\mathbb{H}^{\times}\right) \otimes \operatorname{End}\left(V_{\pi}\right)\right)^{K}$. Thus we have the following commutative diagram

$$
\begin{array}{rlc}
\left(C^{\infty}\left(\mathbb{H}^{\times}\right) \otimes \operatorname{End}\left(V_{\pi}\right)\right)^{K} & \longrightarrow & C^{\infty}((0,1)) \otimes \operatorname{End}_{M}\left(V_{\pi}\right) \\
D, E \downarrow & \downarrow \tilde{D}, \tilde{E} \\
\left(C^{\infty}\left(\mathbb{H}^{\times}\right) \otimes \operatorname{End}\left(V_{\pi}\right)\right)^{K} & \longrightarrow & C^{\infty}((0,1)) \otimes \operatorname{End}_{M}\left(V_{\pi}\right) .
\end{array}
$$

A spherical function $\Phi$ of type $\pi \in \hat{K}$ gives rise to a function $H \in\left(C^{\infty}\left(\mathbb{H}^{\times}\right) \otimes\right.$ $\left.\operatorname{End}\left(V_{\pi}\right)\right)^{K}$ which is an eigenfunction of $D$ (also of $E$ ). In turn such an $H$ corresponds to an $\tilde{H} \in C^{\infty}((0,1)) \otimes \operatorname{End}_{M}\left(V_{\pi}\right)$ which is an eigenfunction of $\tilde{D}$ (also $\tilde{E}$ ) with the extra condition $\lim _{s \rightarrow 1^{-}} \tilde{H}(s)=I$. Conversely if $\tilde{H}$ is such a function then it is the restriction of a unique eigenfunction $H \in\left(C^{\infty}\left(\mathbb{H}^{\times}\right) \otimes \operatorname{End}\left(V_{\pi}\right)\right)^{K}$ of $D$ (also of $E$ ) such that $\lim _{p \rightarrow o} H(p)=I$. From Proposition 3.3, it is clear that $D$ is an elliptic differential operator, thus $H$ can be extended to a $C^{\infty}$-function on $\mathbb{H}$.

Now that the overall strategy has been outlined we go on to give a variety of explicit expressions of $\tilde{D}$ and $\tilde{E}$. See Corollaries 4.6, 4.7, 4.8 as well as (4.3) and (4.4) below.

The proof of the following proposition is included, for completeness, in an Appendix at the end of the paper.

Proposition 4.1. Let $J=\left(\begin{array}{cc}0 & 1 \\ -1 & 0\end{array}\right)$ and $T=\left(\begin{array}{cc}0 & 1 \\ 1 & 0\end{array}\right)$. We have, for $0<r<1$,

$$
\begin{aligned}
& H_{x}(0,0, r)=-\frac{r}{1-r^{2}}(\dot{\pi}(J) \tilde{H}(r)-\tilde{H}(r) \dot{\pi}(J)), \\
& H_{y}(0,0, r)=-\frac{i r}{1-r^{2}}(\dot{\pi}(T) \tilde{H}(r)-\tilde{H}(r) \dot{\pi}(T)),
\end{aligned}
$$

and

$$
\begin{aligned}
H_{x x}(0,0, r)= & -\frac{2 r}{1-r^{2}} \frac{d \tilde{H}}{d r}+\frac{r^{2}}{\left(1-r^{2}\right)^{2}}\left(\dot{\pi}(J)^{2} \tilde{H}(r)+\tilde{H}(r) \dot{\pi}(J)^{2}\right) \\
& -\frac{2 r^{2}}{\left(1-r^{2}\right)^{2}} \dot{\pi}(J) \tilde{H}(r) \dot{\pi}(J) \\
H_{y y}(0,0, r)= & -\frac{2 r}{1-r^{2}} \frac{d \tilde{H}}{d r}-\frac{r^{2}}{\left(1-r^{2}\right)^{2}}\left(\dot{\pi}(T)^{2} \tilde{H}(r)+\tilde{H}(r) \dot{\pi}(T)^{2}\right) \\
& +\frac{2 r^{2}}{\left(1-r^{2}\right)^{2}} \dot{\pi}(T) \tilde{H}(r) \dot{\pi}(T) .
\end{aligned}
$$


Theorem 4.2. We have, for $0<r<1$,

$$
\begin{aligned}
\frac{1}{4} \tilde{D} \tilde{H}(r)= & r^{2} \frac{d^{2} \tilde{H}}{d r^{2}}-\frac{r\left(1+3 r^{2}\right)}{1-r^{2}} \frac{d \tilde{H}}{d r} \\
& +\frac{r^{2}}{\left(1-r^{2}\right)^{2}}\left(\dot{\pi}(J)^{2} \tilde{H}(r)+\tilde{H}(r) \dot{\pi}(J)^{2}-2 \dot{\pi}(J) \tilde{H}(r) \dot{\pi}(J)\right) \\
& -\frac{r^{2}}{\left(1-r^{2}\right)^{2}}\left(\dot{\pi}(T)^{2} \tilde{H}(r)+\tilde{H}(r) \dot{\pi}(T)^{2}-2 \dot{\pi}(T) \tilde{H}(r) \dot{\pi}(T)\right),
\end{aligned}
$$

where $J=\left(\begin{array}{cc}0 & 1 \\ -1 & 0\end{array}\right)$ and $T=\left(\begin{array}{ll}0 & 1 \\ 1 & 0\end{array}\right)$.

Proof. By Proposition 3.3, we have

$$
\frac{1}{4} D H(0,0, r)=H_{x x}(0,0, r)+H_{y y}(0,0, r)+r^{2} H_{r r}(0,0, r)+3 r H_{r}(0,0, r) .
$$

Using Proposition 4.1, the theorem follows.

Theorem 4.3. We have

$$
\begin{aligned}
\tilde{E} \tilde{H}(r)= & -2 r \frac{d \tilde{H}}{d r} \dot{\pi}\left(H_{1}\right)+\frac{4}{1-r^{2}}\left(\dot{\pi}\left(X_{2}\right) \tilde{H}(r)-\tilde{H}(r) \dot{\pi}\left(X_{2}\right)\right) \dot{\pi}\left(X_{1}\right) \\
& -\frac{4 r^{2}}{1-r^{2}}\left(\dot{\pi}\left(X_{1}\right) \tilde{H}(r)-\tilde{H}(r) \dot{\pi}\left(X_{1}\right)\right) \dot{\pi}\left(X_{2}\right)
\end{aligned}
$$

where $H_{1}=\left(\begin{array}{cc}1 & 0 \\ 0 & -1\end{array}\right), X_{1}=\left(\begin{array}{ll}0 & 1 \\ 0 & 0\end{array}\right)$ and $X_{2}=\left(\begin{array}{ll}0 & 0 \\ 1 & 0\end{array}\right)$.

Proof. By Proposition 3.4, we have

$$
E H(0,0, r)=H_{x} \dot{\pi}\left(\begin{array}{cc}
0 & 2 / r \\
2 r & 0
\end{array}\right)+H_{y} \dot{\pi}\left(\begin{array}{cc}
0 & 2 i / r \\
-2 i r & 0
\end{array}\right)+H_{r} \dot{\pi}\left(\begin{array}{cc}
-2 r & 0 \\
0 & 2 r
\end{array}\right) .
$$

Then using Proposition 4.1, we obtain

$$
\begin{aligned}
\tilde{E} \tilde{H}= & -2 r \frac{d \tilde{H}}{d r} \dot{\pi}\left(H_{1}\right)-\frac{2 r}{1-r^{2}}(\dot{\pi}(J) \tilde{H}(r)-\tilde{H}(r) \dot{\pi}(J)) \dot{\pi}\left(\begin{array}{cc}
0 & 1 / r \\
r & 0
\end{array}\right) \\
& +\frac{2 r}{1-r^{2}}(\dot{\pi}(t) \tilde{H}(r)-\tilde{H}(r) \dot{\pi}(t)) \dot{\pi}\left(\begin{array}{cc}
0 & 1 / r \\
-r & 0
\end{array}\right) \\
= & -2 r \frac{d \tilde{H}}{d r} \dot{\pi}\left(H_{1}\right)-\frac{2}{1-r^{2}}(\dot{\pi}(J) \tilde{H}(r)-\tilde{H}(r) \dot{\pi}(J)) \dot{\pi}\left(X_{1}\right) \\
& -\frac{2 r^{2}}{1-r^{2}}(\dot{\pi}(J) \tilde{H}(r)-\tilde{H}(r) \dot{\pi}(J)) \dot{\pi}\left(X_{2}\right)
\end{aligned}
$$




$$
\begin{aligned}
& +\frac{2}{1-r^{2}}(\dot{\pi}(T) \tilde{H}(r)-\tilde{H}(r) \dot{\pi}(T)) \dot{\pi}\left(X_{1}\right) \\
& -\frac{2 r^{2}}{1-r^{2}}(\dot{\pi}(T) \tilde{H}(r)-\tilde{H}(r) \dot{\pi}(T)) \dot{\pi}\left(X_{2}\right) .
\end{aligned}
$$

Now using that $J=X_{1}-X_{2}$ and $T=X_{1}+X_{2}$ the theorem follows.

The theorems above are given in terms of linear transformations. Now we will give the corresponding statements in terms of matrices by choosing an appropriate basis. If $\pi=\pi_{\ell}$ it is well known (see [9, p. 32]) that there exists a basis $\left\{v_{i}\right\}_{i=0}^{\ell}$ of $V_{\pi}$ such that

$$
\begin{array}{ll}
\dot{\pi}\left(H_{1}\right) v_{i}=(\ell-2 i) v_{i}, & \\
\dot{\pi}\left(X_{1}\right) v_{i}=(\ell-i+1) v_{i-1}, & \left(v_{-1}=0\right), \\
\dot{\pi}\left(X_{2}\right) v_{i}=(i+1) v_{i+1}, & \left(v_{\ell+1}=0\right),
\end{array}
$$

where $H_{1}=\left(\begin{array}{cc}1 & 0 \\ 0 & -1\end{array}\right), X_{1}=\left(\begin{array}{ll}0 & 1 \\ 0 & 0\end{array}\right)$ and $X_{2}=\left(\begin{array}{cc}0 & 0 \\ 1 & 0\end{array}\right)$.

Lemma 4.4. The function $\tilde{H}$ is diagonalizable.

Proof. The subgroup $M$ of $K$,

$$
M=\left\{m=\left(\begin{array}{cc}
e^{i t} & 0 \\
0 & e^{-i t}
\end{array}\right): t \in \mathbb{R}\right\},
$$

fixes the points $(0, r)$ in the hyperbolic space $\mathbb{H}=\left\{(z, r): z \in \mathbb{C}, r \in \mathbb{R}^{+}\right\}$. We have $H(k g)=\pi(k) H(g) \pi\left(k^{-1}\right)$, therefore $\tilde{H}(r)=\pi(m) \tilde{H}(r) \pi\left(m^{-1}\right)$. Now since all finite dimensional irreducible $K$-modules are multiplicity free as $M$-modules, $\tilde{H}(r)$, $r>0$, and $\dot{\pi}\left(H_{1}\right)$ diagonalize simultaneously.

We introduce the functions $\tilde{h}_{j}(r)$ by means of the relations

$$
\tilde{H}(r) v_{j}=\tilde{h}_{j}(r) v_{j}
$$

Proposition 4.5. The functions $\tilde{h}_{j}$ defined above satisfy

$$
\tilde{h}_{j}(r)=\tilde{h}_{\ell-j}\left(r^{-1}\right),
$$

for all $0<r<\infty$.

Proof. From $m=\left(\begin{array}{cc}e^{i t} & 0 \\ 0 & e^{-i t}\end{array}\right)=\exp t i H_{1}$ it follows that $\pi(m) v_{j}=e^{i t(\ell-2 j)} v_{j}$. Let $b=\left(\begin{array}{cc}0 & 1 \\ -1 & 0\end{array}\right) \in K$. If $m=\exp t i H_{1}$ we have that $m b=b m^{-1}$, thus $\pi(m) \pi(b) v_{j}=$ $e^{-i t(\ell-2 j)} \pi(b) v_{j}$. Therefore $\pi(b) v_{j}=c_{j} v_{\ell-j}$. Since $b^{2}=\exp \pi i H_{1}$ it follows that $\pi\left(b^{2}\right)=(-1)^{\ell} I$, hence $(-1)^{\ell} v_{j}=c_{j} c_{\ell-j} v_{j}$, so we have

$$
c_{j} c_{\ell-j}=(-1)^{\ell}, \quad \text { for } j=0, \ldots, \ell .
$$


Let $a_{s}=\left(\begin{array}{cc}s & 0 \\ 0 & s^{-1}\end{array}\right) \in G, s>0$. Then

$$
H\left(a_{s}\right)=H\left(p\left(a_{s}\right)\right)=H\left(0, s^{-2}\right)=\tilde{H}\left(s^{-2}\right) .
$$

On the other hand $H\left(a_{s^{-1}}\right)=H\left(b^{-1} a_{s} b\right)=\pi\left(b^{-1}\right) H\left(a_{s}\right) \pi(b)$, thus

$$
\begin{aligned}
\tilde{H}\left(s^{2}\right) v_{j} & =\pi\left(b^{-1}\right) \tilde{H}\left(s^{-2}\right) \pi(b) v_{j}=c_{j} \tilde{h}_{\ell-j}\left(s^{-2}\right) \pi\left(b^{-1}\right) v_{\ell-j} \\
& =(-1)^{\ell} c_{j} c_{\ell-j} \tilde{h}_{\ell-j}\left(s^{-2}\right) v_{j},
\end{aligned}
$$

therefore

$$
\tilde{h}_{j}\left(s^{2}\right)=(-1)^{\ell} c_{j} c_{\ell-j} \tilde{h}_{\ell-j}\left(s^{-2}\right)=\tilde{h}_{\ell-j}\left(s^{-2}\right) .
$$

Corollary 4.6. The function $\tilde{H}(r)=\left(\tilde{h}_{0}(r), \ldots, \tilde{h}_{\ell}(r)\right),(0<r<1)$, satisfies $\left(\frac{1}{4} \tilde{D} \tilde{H}\right)(r)=\lambda \tilde{H}(r)$ if and only if

$$
\begin{aligned}
r^{2} \tilde{h}_{i}^{\prime \prime} & -\frac{r\left(1+3 r^{2}\right)}{1-r^{2}} \tilde{h}_{i}^{\prime} \\
& +\frac{4 r^{2}}{\left(1-r^{2}\right)^{2}}\left(i(\ell-i+1)\left(\tilde{h}_{i-1}-\tilde{h}_{i}\right)+(i+1)(\ell-i)\left(\tilde{h}_{i+1}-\tilde{h}_{i}\right)\right)=\lambda \tilde{h}_{i}
\end{aligned}
$$

for all $i=0, \ldots, \ell$.

Corollary 4.7. The function $\tilde{H}(r)=\left(\tilde{h}_{0}(r), \ldots, \tilde{h}_{\ell}(r)\right),(0<r<1)$, satisfies $(\tilde{E} \tilde{H})(r)=\mu \tilde{H}(r)$ if and only if

$$
-2 r(\ell-2 i) \tilde{h}_{i}^{\prime}+\frac{4 i(\ell-i+1)}{1-r^{2}}\left(\tilde{h}_{i-1}-\tilde{h}_{i}\right)-\frac{4 r^{2}(i+1)(\ell-i)}{1-r^{2}}\left(\tilde{h}_{i+1}-\tilde{h}_{i}\right)=\mu \tilde{h}_{i}
$$

for all $i=0, \ldots, \ell$.

We introduce the change of variable $t=r^{2}$ and we put $H(t)=\tilde{H}(\sqrt{t})$ and $h_{i}(t)=\tilde{h}_{i}(\sqrt{t})$. The differential operators $\tilde{D}$ and $\tilde{E}$ are converted into new differential operators $D$ and $E$. At this point there is a slight abuse of notation, since $D$ and $E$ were used earlier to denote operators on $\mathbb{H}$. We get the following expressions for these new differential operators $D$ and $E$.

Corollary 4.8. For $0<t<1$, the function $\tilde{H}$ satisfies $(D H)(t)=4 \lambda H(t)$ and $(E H)(t)=\mu H(t)$ if and only if

$$
\begin{gathered}
4 t^{2} h_{i}^{\prime \prime}-\frac{8 t^{2}}{1-t} h_{i}^{\prime}+\frac{4 t}{(1-t)^{2}} i(\ell-i+1)\left(h_{i-1}-h_{i}\right) \\
+\frac{4 t}{(1-t)^{2}}(i+1)(\ell-i)\left(h_{i+1}-h_{i}\right)=\lambda h_{i},
\end{gathered}
$$

and

$$
\begin{gathered}
4(\ell-2 i) t h_{i}^{\prime}+\frac{4}{1-t} i(\ell-i+1)\left(h_{i-1}-h_{i}\right) \\
-\frac{4 t}{1-t}(i+1)(\ell-i)\left(h_{i+1}-h_{i}\right)=\mu h_{i},
\end{gathered}
$$

respectively. 
In matrix notation, the differential operators $D$ and $E$ are given by

$$
\begin{aligned}
& D H=4 t^{2} H^{\prime \prime}-\frac{8 t^{2}}{1-t} H^{\prime}+\frac{4 t}{(1-t)^{2}}\left(C_{0}+C_{1}\right) H . \\
& E H=-4 t A_{0} H^{\prime}+\frac{4}{1-t} C_{0} H-\frac{4 t}{1-t} C_{1} H .
\end{aligned}
$$

Here $H$ denotes the column vector $H=\left(h_{0}, \ldots, h_{\ell}\right)$ and the matrices are given by:

$$
\begin{aligned}
& A_{0}=\sum_{i=0}^{\ell}(\ell-2 i) E_{i i}, \\
& C_{0}=\sum_{i=0}^{\ell} i(\ell-i+1)\left(E_{i, i-1}-E_{i, i}\right), \\
& C_{1}=\sum_{i=0}^{\ell}(i+1)(\ell-i)\left(E_{i, i+1}-E_{i, i}\right) .
\end{aligned}
$$

Remark 4.9. The function $\Phi \in C^{\infty}(G) \otimes \operatorname{End}\left(V_{\pi}\right)$ satisfies $(4 \bar{\Omega}) \Phi=\tilde{\lambda} \Phi$ and $(\Omega-\bar{\Omega}) \Phi=\tilde{\mu} \Phi$ if and only if the function $H=H(t)$ associated with $\Phi$ satisfies $D H=\lambda H$ and $E H=\mu H$, with

$$
\tilde{\lambda}=4 \lambda \text { and } \tilde{\mu}=\mu+\ell(\ell+2),
$$

(see Proposition 3.1).

\section{Eigenfunctions of $D$}

The goal of Secs. 5 and 6 is:

(i) to describe all solutions to $D H=\lambda H, E H=\mu H, 0<t<1$,

(ii) to identify among these, those $H$ that have a limit as $t \rightarrow 1^{-}$. These correspond precisely to the spherical functions, as seen in Sec. 4.

We are interested in considering first the functions $H:(0,1) \longrightarrow \mathbb{C}^{\ell+1}$ such that $D H=\lambda H, \lambda \in \mathbb{C}$. It is well known that such eigenfunctions are analytic functions on the open interval $(0,1)$ and that the dimension of the corresponding eigenspace $V_{\lambda}$ is $2(\ell+1)$. This space will be decomposed in different ways as a direct sum of $\ell+1$ dimensional spaces stable under E. See Propositions 5.6, 5.9 and 5.10. The action of $E$ on these spaces will be taken up in Sec. 6 .

To determine the eigenfunctions of the differential operator

$$
D H=4 t^{2} H^{\prime \prime}-\frac{8 t^{2}}{1-t} H^{\prime}+\frac{4 t}{(1-t)^{2}}\left(C_{0}+C_{1}\right) H
$$

for $0<t<1$, it is necessary to take a close look at it and this is the purpose of the next proposition.

Since the $(\ell+1) \times(\ell+1)$ matrix $C_{0}+C_{1}$ is not a diagonal matrix, the equation $D H=\lambda H$ is a coupled system of $\ell+1$ second order differential equations in the 
components $\left(h_{0}, \ldots, h_{\ell}\right)$ of $H$. But fortunately the matrix $C_{0}+C_{1}$ is a symmetric one, thus diagonalizable.

Proposition 5.1. The matrix $C_{0}+C_{1}$ is diagonalizable. Moreover the eigenvalues are $-j(j+1)$ for $0 \leq j \leq \ell$ and the corresponding eigenvectors are given by $u_{j}=$ $\left(u_{0, j}, \ldots, u_{\ell, j}\right)$ where

$$
u_{i, j}={ }_{3} F_{2}\left(\begin{array}{c}
-j,-i, j+1 \\
1,-\ell
\end{array} ; 1\right),
$$

an instance of the Hahn orthogonal polynomials.

Proof. We shall see that

$$
\left(C_{0}+C_{1}\right) u_{j}=-j(j+1) u_{j} \text { for all } 0 \leq j \leq \ell .
$$

The matrix $C_{0}+C_{1}$ is given by

$$
\sum_{i=0}^{\ell} i(\ell-i+1) E_{i, i-1}-(i(\ell-i+1)+(i+1)(\ell-i)) E_{i, i}+(i+1)(\ell-i) E_{i, i+1} .
$$

Then the $i$ th-component of (5.2) is given by

$$
\begin{gathered}
i(\ell-i+1) u_{i-1, j}-(i(\ell-i+1)+(i+1)(\ell-i)) u_{i, j} \\
+(i+1)(\ell-i) u_{i+1, j}=-j(j+1) u_{i, j} .
\end{gathered}
$$

This allows us to recognize the eigenvectors of $C_{0}+C_{1}$ as an instance of the Hahn polynomials.

For real numbers $\alpha, \beta>-1$, and for a positive integer $N$ the Hahn polynomials $Q_{n}(x)=Q_{n}(x ; \alpha, \beta, N)$ are defined by

$$
Q_{n}(x)={ }_{3} F_{2}\left(\begin{array}{c}
-n,-x, n+\alpha+\beta+1 \\
\alpha+1,-N+1
\end{array} ; 1\right), \quad \text { for } n=0,1, \ldots, N-1 .
$$

These Hahn polynomials are examples of orthogonal polynomials and hence satisfy a three term recursion relation, see [10, (1.3)] or [1]. If one takes $\alpha=\beta=0, x=i$, $N=\ell+1, n=j$ we obtain

$$
u_{i, j}=Q_{j}(i)={ }_{3} F_{2}\left(\begin{array}{c}
-j,-i, j+1 \\
1,-\ell
\end{array} ; 1\right) .
$$

Since all the eigenvalues are different we have that $C_{0}+C_{1}$ is diagonalizable.

Let $U=\left(u_{i, j}\right)$ be the matrix defined by (5.1). Then

$$
U\left(-\sum_{j=0}^{\ell} j(j+1) E_{j j}\right) U^{-1}=C_{0}+C_{1}
$$


If we define $\check{H}(t)=U^{-1} H(t)$, we see that the $i$ th-component $\check{h}_{i}(t)$ of $\check{H}(t)$ satisfies

$$
4 t^{2} \check{h}_{i}^{\prime \prime}(t)-\frac{8 t^{2}}{1-t} \check{h}_{i}^{\prime}(t)-4 i(i+1) \frac{t}{(1-t)^{2}} \check{h}_{i}(t)-\lambda \check{h}_{i}(t)=0
$$

if and only if $D H=\lambda H$.

Recall that

$$
V_{\lambda}=\{H=H(t): D H=\lambda H(t), 0<t<1\} .
$$

Suppose that $0 \neq H \in V_{\lambda}$ is of the form $H(t)=t^{p} F(t)$ with $F$ analytic at $t=0$ and $p \in \mathbb{C}$. In such a case we may assume that $F(0) \neq 0$. We first note the following relation between $\lambda$ and $p$.

Lemma 5.2. If $H=t^{p} F(t)$ with $F$ analytic at $t=0$, and $F(0) \neq 0$ satisfies $D H=\lambda H$, then $\lambda=4 p(p-1)$.

Proof. Let $F(t)=\sum_{j=0}^{\infty} t^{j} F_{j}$, then $F$ satisfies

$$
\begin{aligned}
& 4 t^{2} F^{\prime \prime}+\frac{8 t}{1-t}(p-(p+1) t) F^{\prime}+\frac{4 t}{(1-t)^{2}}\left(-2 p(1-t)+C_{0}+C_{1}\right) F \\
& \quad+4 p(p-1) F-\lambda F=0 .
\end{aligned}
$$

If we multiply the above expression by $(1-t)^{2}$ and set $t=0$, we obtain $(4 p(p-1)-\lambda) F(0)=0$ and the lemma follows.

Observe that the function $\lambda(p)=4 p(p-1)$ satisfies $\lambda(p)=\lambda(1-p)$. Given $\lambda \in \mathbb{C}$, if $p \in \mathbb{C}$ satisfies $\lambda=4 p(p-1)$ let

$$
V(p)=\left\{H \in V_{\lambda}: H(t)=t^{p} F(t), F \text { analytic at } t=0\right\} .
$$

We note that if $H(t)=t^{p} F(t) \in V(p)$, with $F(t)=\sum_{j=0}^{\infty} t^{j} F_{j}$ then the vector coefficients $F_{j}$ satisfy the three term recursion relation

$$
\begin{gathered}
\left.j(j-1+2 p) F_{j}-\left(2 p(2 j-1)+2(j-1)^{2}-C_{0}-C_{1}\right)\right) F_{j-1} \\
+(j-1)(2 p+j-2) F_{j-2}=0, \quad j \geq 1 .
\end{gathered}
$$

Proposition 5.3. Let $H \in V_{\lambda}, \lambda=4 p(p-1)$ and let $\check{H}(t)=U^{-1} H(t)$. If we write

$$
\check{H}(t)=\frac{t^{p}}{(1-t)^{\ell+1}} \check{P}(t),
$$

then the ith-component $\check{P}_{i}=\check{P}_{i}(t)$ of $\check{P}$ is of the form $\check{P}_{i}(t)=(1-t)^{\ell-i} R_{i}(t)$ where $R_{i}$ is a solution of the differential equation

$$
t(1-t) R_{i}^{\prime \prime}+\left(2 p-(2(p-i) t) R_{i}^{\prime}+i(2 p-i-1) R_{i}=0 .\right.
$$

Notice that (5.7) is nothing but the Gauss' hypergeometric equation with parameters $a=-i, b=2 p-i-1, c=2 p$. 
Proof. If $H \in V_{\lambda}$ we can write $H(t)=\frac{t^{p}}{(1-t)^{\ell+1}} P(t)$ with $P$ analytic on the interval $0<t<1$. Then it is easy to verify that $P$ satisfies

$$
\begin{aligned}
4 t^{2} P^{\prime \prime} & +\frac{4 t}{1-t}(2 p-2(p-\ell) t) P^{\prime} \\
& +\frac{4 t}{(1-t)^{2}}\left(\ell(\ell+1) t+2 p \ell(1-t)+C_{0}+C_{1}\right) P=0 .
\end{aligned}
$$

If we define the function $\check{P}=U^{-1} P$ it follows from (5.8) that the $i$ th-component $\check{P}_{i}$ of $\check{P}$ satisfies the following differential equation

$$
\begin{aligned}
& t(1-t) \check{P}_{i}^{\prime \prime}+(2 p-2(p-\ell) t) \check{P}_{i}^{\prime} \\
& \quad+\frac{1}{(1-t)}(\ell(\ell+1) t+2 p \ell(1-t)-i(i+1)) \check{P}_{i}=0 .
\end{aligned}
$$

We can also set $\check{P}_{i}=(1-t)^{\ell-i} R_{i}$ and then it is easy to verify that $R_{i}$ satisfies

$$
t(1-t) R_{i}^{\prime \prime}+\left(2 p-(2(p-i) t) R_{i}^{\prime}+i(2 p-i-1) R_{i}=0 .\right.
$$

This finishes the proof of the proposition.

Proposition 5.4. If $\lambda=4 p(p-1)$ with $2 p \in \mathbb{C}$ not an integer, and $H \in V(p)$, $H \neq 0$, then

$$
H(t)=\frac{t^{p}}{(1-t)^{\ell+1}} P(t),
$$

where $P=P(t)$ is a polynomial function of degree $\ell$. More precisely $P(t)=U \check{P}(t)$ where the ith-component of $\check{P}$ is

$$
\check{P}_{i}=\alpha_{i}(1-t)_{2}^{\ell-i} F_{1}\left(\begin{array}{c}
-i, 2 p-i-1 \\
2 p
\end{array} ;\right)
$$

with $\alpha_{i} \in \mathbb{C}$.

Proof. By hypothesis, for any $H \in V(p)$, we may write $H(t)=\frac{t^{p}}{(1-t)^{\ell+1}} P(t)$ with $P$ analytic at $t=0$. Let $\check{H}=U^{-1} H$ and $\check{P}=U^{-1} P$. Then by Proposition 5.3, the $i$ th-component $\check{P}_{i}$ of $\check{P}$ is of the form $\check{P}_{i}=(1-t)^{\ell-i} R_{i}$ where $R_{i}$ satisfies the Gauss' hypergeometric equation (5.7). Moreover $R_{i}$ is analytic at $t=0$.

Since $2 p \notin \mathbb{Z}$ it is easy to verify that a basis of the solutions of (5.7) is given by the functions:

$$
{ }_{2} F_{1}\left(\begin{array}{c}
-i, 2 p-i-1 \\
2 p
\end{array} t\right), \quad t^{1-2 p}{ }_{2} F_{1}\left(\begin{array}{c}
-i,-2 p-i+1 \\
2-2 p
\end{array} ; t\right) .
$$

Therefore

$$
R_{i}=\alpha_{i 2} F_{1}\left(\begin{array}{c}
-i, 2 p-i-1 \\
2 p
\end{array} ; t\right)
$$


for some $\alpha_{i} \in \mathbb{C}$. Hence each $\check{P}_{i}$ is a polynomial function of degree $\ell$, whenever $\alpha_{i} \neq 0$. Since $P=U \check{P}$ the assertions made in the statement of the proposition are now clear.

Corollary 5.5. If $2 p \in \mathbb{C}$ is not an integer then $\operatorname{dim} V(p)=\ell+1$.

Proposition 5.6. If $2 p \in \mathbb{C}$ is not an integer and $\lambda=4 p(p-1)$, then

$$
V_{\lambda}=V(p) \oplus V(1-p)
$$

Proof. Let $H \in V(p) \cap V(1-p)$. If $H \neq 0$ then $H=t^{p} F(t)=t^{1-p} G(t)$ with $F$ and $G$ analytic at $t=0$. Since $2 p \notin \mathbb{Z}$ from (5.6) we get $F(0) \neq 0$. Thus $t^{2 p-1}=\frac{G(t)}{F(t)}$ is analytic at $t=0$, but $t^{2 p-1}$ has a ramification point at $t=0$, because $2 p \notin \mathbb{Z}$. This contradiction proves that $V(p) \cap V(1-p)=0$.

Since $\operatorname{dim} V(p)=\operatorname{dim} V(1-p)=\ell+1$ (Corollary 5.5) the proof of the proposition is completed.

When $2 p \in \mathbb{Z}$ the situation is completely different as shown in the next proposition. The reader may choose to skip the computation of $\operatorname{dim} V(1-p)$ in (i) and of $\operatorname{dim} V(p)$ in (ii).

Proposition 5.7. Let $2 p \in \mathbb{Z}$ and $\lambda=4 p(p-1)$.

(i) If $2 p \geq 1$ then we have $V(p) \subset V(1-p) \subset V_{\lambda}, \operatorname{dim} V(p)=\ell+1$ and $\operatorname{dim} V(1-p)=\min \{2(\ell+1), \ell+2 p\}$.

(ii) If $2 p \leq 0$ then $V(1-p) \subset V(p) \subset V_{\lambda}$, $\operatorname{dim} V(1-p)=\ell+1$ and $\operatorname{dim} V(p)=$ $\min \{2(\ell+1), \ell+2-2 p\}$.

Proof. Let us first observe that (i) and (ii) are equivalent by changing $p$ by $1-p$. Thus we can assume that $2 p \geq 1$.

If $H \in V(p)$ then $H(t)=t^{p} F(t)=t^{1-p}\left(t^{2 p-1} F(t)\right)$ with $t^{2 p-1} F(t)$ analytic at $t=0$. This proves that $V(p) \subset V(1-p)$.

If $H \in V_{\lambda}$ and $\check{H}=U^{-1} H$, then by Proposition 5.3, the $i$ th-component $\check{h}_{i}$ of $\check{H}$ is of the form $\check{h}_{i}=\frac{t^{p}}{(1-t)^{i+1}} R_{i}$ where $R_{i}$ is a solution of the Gauss' equation with parameters $a=-i, b=2 p-i-1, c=2 p$.

A convenient basis of solutions of this Gauss' equation for $0<t<1$, is given by the functions (see [2, Sec. 2.2.2, Case 22])

$$
{ }_{2} F_{1}\left(\begin{array}{c}
-i, 2 p-i-1 \\
2 p
\end{array} t\right), \quad t^{1-2 p}{ }_{2} F_{1}\left(\begin{array}{c}
-i,-2 p-i+1 \\
2-2 p
\end{array} ; t\right),
$$

whenever $0 \leq i<2 p-1$. On the other hand if $2 p-1 \leq i \leq \ell$ a basis of solutions is given by the functions (see [2, Sec. 2.2.2, Case 23])

$$
{ }_{2} F_{1}\left(\begin{array}{c}
-i, 2 p-i-1 \\
2 p
\end{array} t\right), \quad(1-t)^{2 i+1}{ }_{2} F_{1}\left(\begin{array}{c}
i+1,2 p+i \\
2 i+2
\end{array} ; 1-t\right) .
$$


In both cases it is easy to verify that these functions solve Gauss' equation and are linearly independent. In both cases the second elements of these bases are not analytic at $t=0$ while the other one is. In fact, in the case $2 p>i+1$ the function $t^{1-2 p}{ }_{2} F_{1}(-i,-2 p-i+1,2-2 p ; t)$ has a pole at $t=0$ of order $2 p-1>0$. When $1 \leq 2 p \leq i+1$ we have the following identity ([2, Chap. $2,(4)])$ :

$$
{ }_{2} F_{1}\left(\begin{array}{c}
i+1,2 p+i \\
2 i+2
\end{array} ; z\right)=c\left(\frac{d}{d z}\right)^{i+2 p-1}\left((1-z)^{i}\left(\frac{d}{d z}\right)^{i+1-2 p}\left(-z^{-1} \log (1-z)\right)\right) \text {. }
$$

From this it is clear that the function ${ }_{2} F_{1}(i+1,2 p+i, 2 i+2 ; z)$ is not analytic at $z=1$ because $\log (1-z)$ has a ramification point at $z=1$. Thus at $t=0$, $(1-t)^{2 i+1}{ }_{2} F_{1}(i+1,2 p+i, 2 i+2 ; 1-t)$ is not analytic.

If $H \in V(p)$ then $R_{i}$ is analytic at $t=0$, therefore

$$
R_{i}=\alpha_{i 2} F_{1}\left(\begin{array}{c}
-i, 2 p-i-1 \\
2 p
\end{array} ; t\right)
$$

for some $\alpha_{i} \in \mathbb{C}$. Thus $\operatorname{dim} V(p)=\ell+1$.

Now we compute the dimension of $V(1-p)$. We observe that by Proposition 5.3 for $H \in V(\lambda)$, the $i$ th-component $\check{h}_{i}$ of $\check{H}$ is of the form $\check{h}_{i}=\frac{t^{p}}{(1-t)^{i+1}} R_{i}$. We can also obtain that $\check{h}_{i}(t)=\frac{t^{1-p}}{(1-t)^{i+1}} S_{i}$, where $S_{i}$ is a solution of the Gauss' equation with parameters $a=-i, b=-2 p-i+1, c=2-2 p$. We have the relation $S_{i}=t^{2 p-1} R_{i}$. Therefore a basis of the solutions of this Gauss' equation, for $0<t<1$ is given by

$$
t^{2 p-1}{ }_{2} F_{1}\left(\begin{array}{c}
-i, 2 p-i-1 \\
2 p
\end{array} t t\right), \quad{ }_{2} F_{1}\left(\begin{array}{c}
-i,-2 p-i+1 \\
2-2 p
\end{array} ; t\right)
$$

whenever $0 \leq i<2 p-1$. On the other hand if $2 p-1 \leq i \leq \ell$ a basis of solutions is given by

$$
t_{2}^{2 p-1} F_{1}\left(\begin{array}{c}
-i, 2 p-i-1 \\
2 p
\end{array} ; t\right), \quad t^{2 p-1}(1-t)^{2 i+1}{ }_{2} F_{1}\left(\begin{array}{c}
i+1,2 p+i \\
2 i+2
\end{array} ; 1-t\right) .
$$

In the first case, both solutions are analytic at $t=0$, moreover they are polynomials, while in the second case, the first solution is analytic and the other one is not.

If $H \in V(1-p)$ then $S_{i}$ is analytic at $t=0$, therefore we have for $0 \leq i<2 p-1$ that

$$
S_{i}=\alpha_{i} t_{2}^{2 p-1} F_{1}\left(\begin{array}{c}
-i, 2 p-i-1 \\
2 p
\end{array} ; t\right)+\beta_{i 2} F_{1}\left(\begin{array}{c}
-i,-2 p-i+1 \\
2-2 p
\end{array} ; t\right)
$$

for some $\alpha_{i}, \beta_{i} \in \mathbb{C}$. For $2 p-1 \leq i \leq \ell$ we have

$$
S_{i}=\alpha_{i} t^{2 p-1}{ }_{2} F_{1}\left(\begin{array}{c}
-i, 2 p-i-1 \\
2 p
\end{array} ; t\right),
$$

for some $\alpha_{i} \in \mathbb{C}$. From the description of the solutions it follows that $\operatorname{dim} V(1-p)=$ $\min \{2(\ell+1), \ell+2 p\}$. 
For any $p \in \mathbb{C}$ such that $\operatorname{dim} V(p)=\ell+1$ a useful complement of $V(p)$ in $V_{\lambda}$ will turn out to be the space $W_{\lambda}$ introduced below.

A spherical function of type $\pi_{\ell} \in \hat{K}$ gives rise to a function $H \in V_{\lambda}$ such that $\lim _{t \rightarrow 1^{-}} H(t)=(1, \ldots, 1)$. This leads us to consider the linear space

$$
W_{\lambda}=\left\{H \in V_{\lambda}: \lim _{t \rightarrow 1^{-}} H(t) \text { is finite }\right\} .
$$

We start with a useful lemma. The first two cases considered in the hypothesis will be used in Propositions 5.10 and 5.9, respectively.

Lemma 5.8. If $2 p \in \mathbb{C}-\mathbb{Z}$, or $2 p \in \mathbb{Z}$ and $2 p>i+1$, or $2 p \in \mathbb{Z}$ and $2 p<-i+1$, then we have

$$
\begin{gathered}
\frac{(i+1)_{i+1}}{(1-2 p)_{i+1}} F_{1}\left(\begin{array}{c}
-i, 2 p-i-1 \\
2 p
\end{array} ; t\right)+\frac{(i+1)_{i+1}}{(2 p-1)_{i+1}} t^{1-2 p}{ }_{2} F_{1}\left(\begin{array}{c}
-i,-2 p-i+1 \\
2-2 p
\end{array} ; t\right) \\
=t^{1-2 p}(1-t)^{2 i+1}{ }_{2} F_{1}\left(\begin{array}{c}
i+1, i+2-2 p \\
2 i+2
\end{array} 1-t\right) .
\end{gathered}
$$

Proof. Let us first assume that $2 p \in \mathbb{C}-\mathbb{Z}$ and $\operatorname{Re}(2 p)>1$, or that $2 p \in \mathbb{Z}$ and $2 p>i+1$. Under this hypothesis on $p$ the hypergeometric equation with parameters $a=-i, b=2 p-i-1$ and $c=2 p$, as we pointed out before, has a basis of solutions given by (5.10). In general we have that $t^{1-c}(1-t)^{c-a-b}{ }_{2} F_{1}(1-a, 1-b, c+1-$ $a-b ; 1-t)$ is a solution of the hypergeometric equation with parameters $a, b, c$. So, we get

$$
\begin{aligned}
& (1-t)^{2 i+1}{ }_{2} F_{1}\left(\begin{array}{c}
i+1, i+2-2 p \\
2 i+2
\end{array} ; 1-t\right) \\
& \quad=A t^{2 p-1}{ }_{2} F_{1}\left(\begin{array}{c}
-i, 2 p-i-1 \\
2 p
\end{array} ; t\right)+B_{2} F_{1}\left(\begin{array}{c}
-i,-2 p-i+1 \\
2-2 p
\end{array} ; t\right) .
\end{aligned}
$$

We let $t \rightarrow 0^{+}$. Since $\operatorname{Re}(2 p)>1$, we have that $\lim _{t \rightarrow 0^{+}} t^{2 p-1}=0$ and that the function ${ }_{2} F_{1}(i+1, i+2-2 p, 2 i+2 ; 1-t)$ converges when $t \rightarrow 0$ to the value ${ }_{2} F_{1}(i+1, i+2-2 p, 2 i+2 ; 1)=\frac{\Gamma(2 i+2) \Gamma(2 p-1)}{\Gamma(i+1) \Gamma(i+2 p)}$. (See, for example [1, Theorem 2.2.2]). Thus

$$
B={ }_{2} F_{1}\left(\begin{array}{c}
i+1, i+2-2 p \\
2 i+2
\end{array} ; 1\right)=\frac{(i+1)_{i+1}}{(2 p-1)_{i+1}} .
$$

Now by taking $\lim _{t \rightarrow 1}$ we have

$$
\begin{aligned}
0 & =A_{2} F_{1}\left(\begin{array}{c}
-i, 2 p-i-1 \\
2 p
\end{array} ; 1\right)+B_{2} F_{1}\left(\begin{array}{c}
-i,-i+1-2 p \\
2-2 p
\end{array} ; 1\right) \\
& =A \frac{(i+1)_{i}}{(2 p)_{i}}+B \frac{(i+1)_{i}}{(2-2 p)_{i}} .
\end{aligned}
$$

From here we can easily deduce that $A=\frac{(i+1)_{i+1}}{(1-2 p)_{i+1}}$. 
Now we realize that (5.12) is invariant under the map $p \mapsto 1-p$, by Euler's transformation (see $[1$, Theorem 2.2.5]). Thus (5.12) also holds when $2 p \in \mathbb{C}-\mathbb{Z}$ and $\operatorname{Re}(2 p)<1$, or when $2 p \in \mathbb{Z}$ and $2 p<-i+1$. By analytic continuation on $p$ the validity of $(5.12)$ will be extended to $\operatorname{Re}(2 p)=1$ but $2 p \neq 1$. In fact the left hand side of (5.12) is an analytic function of $p$ for $2 p \in \mathbb{C}-\mathbb{Z}$ if $0<t<1$. On the other hand it is well known that $\frac{1}{\Gamma(c)}{ }_{2} F_{1}(a, b, c ; x)$ is an entire function of $a, b, c$ if $|x|<1$ (see [1, p. 65]). Thus the right hand side of (5.12) is an entire function of $p$ if $0<t<1$. This completes the proof of the lemma.

Proposition 5.9. Let $2 p$ be an integer and let $\lambda=4 p(p-1)$. We have

(i) If $2 p \geq 1$ then $V_{\lambda}=V(p) \oplus W_{\lambda}$.

(ii) If $2 p \leq 0$ then $V_{\lambda}=V(1-p) \oplus W_{\lambda}$.

Proof. Let us first observe that (i) and (ii) are equivalent by changing $p$ by $1-p$. Thus let us assume that $2 p \geq 1$.

Let $H \in V_{\lambda}, \lambda=4 p(p-1)$ and $\check{H}=U^{-1} H$, then by Proposition 5.3, the $i$ th-component $\breve{h}_{i}$ of $\check{H}$ is of the form $\check{h}_{i}=\frac{t^{p}}{(1-t)^{i+1}} R_{i}$ where $R_{i}$ is a solution of the Gauss' equation (5.7). We also have mentioned that a basis of solutions of this equation is given by (5.10), when $0 \leq i<2 p-1$ and by (5.11) if $2 p-1 \leq i \leq \ell$. Therefore for any $H \in V_{\lambda}$ the $i$ th-component of $\check{H}$ is

$\check{h}_{i}=\alpha_{i} \frac{t^{p}}{(1-t)^{i+1}}{ }_{2} F_{1}\left(\begin{array}{c}-i, 2 p-i-1 \\ 2 p\end{array} ; t\right)+\beta_{i} \frac{t^{1-p}}{(1-t)^{i+1}}{ }_{2} F_{1}\left(\begin{array}{c}-i,-2 p-i+1 \\ 2-2 p\end{array} ; t\right)$. whenever, $0 \leq i<2 p-1$ and if $2 p-1 \leq i \leq \ell$ then

$\check{h}_{i}=\alpha_{i} \frac{t^{p}}{(1-t)^{i+1}}{ }_{2} F_{1}\left(\begin{array}{c}-i, 2 p-i-1 \\ 2 p\end{array} ; t\right)+\beta_{i} t^{p}(1-t)^{i}{ }_{2} F_{1}\left(\begin{array}{c}i+1,2 p+i \\ 2 i+2\end{array} ; 1-t\right)$, for some $\alpha_{i}, \beta_{i} \in \mathbb{C}$.

In both cases we have seen in the proof of Proposition 5.7 that the functions $H \in V(p)$ have $\beta_{i}=0$, for all $i=0, \ldots, \ell$. In particular $\check{h}_{i}$ diverges when $t \rightarrow 1^{-}$. Therefore we have that $V(p) \cap W_{\lambda}=0$.

We recall that a function $H \in W_{\lambda}$ corresponds to functions $\check{h}_{i}$ which have a finite limit when $t \rightarrow 1^{-}$.

In the case $2 p-1 \leq i \leq \ell$ the second summand in $\check{h}_{i}$

$$
\beta_{i} t^{p}(1-t)_{2}^{i} F_{1}\left(\begin{array}{c}
i+1,2 p+i \\
2 i+2
\end{array} ; 1-t\right)
$$

is convergent at $t=1$, while the first one is not if $\alpha_{i} \neq 0$. Therefore $\check{h}_{i}$ corresponds to a function $H \in W_{\lambda}$ if and only if $\alpha_{i}=0$.

When $0 \leq i<2 p-1$, by Lemma 5.8, if we choose $\alpha_{i}=a_{i} \frac{(i+1)_{i+1}}{(1-2 p)_{i+1}}$ and $\beta_{i}=a_{i} \frac{(i+1)_{i+1}}{(2 p-1)_{i+1}},\left(a_{i} \in \mathbb{C}\right)$, we get

$$
\check{h}_{i}(t)=a_{i} t^{1-p}(1-t)_{2}^{i} F_{1}\left(\begin{array}{c}
i+1, i+2-2 p \\
2 i+2
\end{array} ; 1-t\right)
$$

which is convergent at $t=1$. 
Therefore $\operatorname{dim} W_{\lambda}=\ell+1$ and since $\operatorname{dim} V(p)=\ell+1$ (Proposition 5.7) the proof is completed.

Recall that for $2 p \notin \mathbb{Z}$ we had $V_{\lambda}=V(p) \oplus V(1-p)$. When $2 p \in \mathbb{Z}$ this decomposition fails but can be replaced by one of the two in Proposition 5.9. Returning to the case $2 p \notin \mathbb{Z}$ we have the following proposition.

Proposition 5.10. If $2 p \in \mathbb{C}$ is not an integer and $\lambda=4 p(p-1)$ then

$$
V_{\lambda}=V(p) \oplus W_{\lambda}=V(1-p) \oplus W_{\lambda} .
$$

Proof. Let $H \in V_{\lambda}, \lambda=4 p(p-1)$ and $\check{H}=U^{-1} H$, then by Proposition 5.3, the $i$ th-component $\check{h}_{i}$ of $\check{H}$ is of the form $\check{h}_{i}=\frac{t^{p}}{(1-t)^{i+1}} R_{i}$ where $R_{i}$ is a solution of the Gauss' equation (5.7). Since $2 p \notin \mathbb{Z}$ a basis of the solutions of this equation is given by (5.9). Therefore for any $H \in V_{\lambda}$ the $i$ th-component of $\check{H}$ is

$\check{h}_{i}=\alpha_{i} \frac{t^{p}}{(1-t)^{i+1}}{ }_{2} F_{1}\left(\begin{array}{c}-i, 2 p-i-1 \\ 2 p\end{array} ; t\right)+\beta_{i} \frac{t^{1-p}}{(1-t)^{i+1}}{ }_{2} F_{1}\left(\begin{array}{c}-i,-2 p-i+1 \\ 2-2 p\end{array} ; t\right)$.

Now the proof follows in the same way as in Proposition 5.9.

Corollary 5.11. Let $H \in W_{\lambda}, \lambda=4 p(p-1)$ with $\operatorname{Re}(2 p) \geq 1$ and let $\check{H}=U^{-1} H$. Then the ith-component $\check{h}_{i}$ of $\check{H}$ is of the following form:

(i) If $2 p \notin \mathbb{Z}$ or $2 p$ is an integer and $0 \leq i<2 p-1$ there exists $a_{i} \in \mathbb{C}$ such that

$$
\check{h}_{i}=a_{i} t^{1-p}(1-t)_{2}^{i} F_{1}\left(\begin{array}{c}
i+1, i+2-2 p \\
2 i+2
\end{array} ; 1-t\right) .
$$

(ii) If $2 p$ is an integer and $2 p-1 \leq i \leq \ell$ then

$$
\check{h}_{i}=a_{i} t^{p}(1-t)_{2}^{i} F_{1}\left(\begin{array}{c}
i+1,2 p+i \\
2 i+2
\end{array} ; 1-t\right)
$$

for some constant $a_{i} \in \mathbb{C}$.

Proof. Instance (i) consists of two cases. The first one follows from Proposition 5.10 combined with Lemma 5.8. For the second, see (5.14). Instance (ii) was obtained in (5.13).

Corollary 5.12. For all $p \in \mathbb{C}$ we have that $\operatorname{dim} W_{\lambda}=\ell+1$.

In the next section the asymptotic behavior of $H \in V_{\lambda}$ when $t \rightarrow 0^{+}$will play a fundamental role.

Proposition 5.13. If $H \in V(p), p \in \mathbb{C}$, then $\lim _{t \rightarrow 0^{+}} t^{-p} H(t)$ exists and is finite.

Proof. The assertion follows directly from the definition of $V(p)$ in (5.5). 
Proposition 5.14. Let $H \in W_{\lambda}, \lambda=4 p(p-1)$ with $\operatorname{Re}(2 p)>1$. Then $\lim _{t \rightarrow 0^{+}} t^{p-1} H(t)$ exists and is finite.

Proof. Observe that if $\check{H}=U^{-1} H$ then it is equivalent to prove the existence of $\lim _{t \rightarrow 0^{+}} t^{p-1} \check{H}(t)$.

In the case $2 p \notin \mathbb{Z}$ or $2 p$ an integer with $2 p>i+1$ we have, by Corollary 5.11 (i), that

$$
\check{h}_{i}=a_{i} t^{1-p}(1-t)_{2}^{i} F_{1}\left(\begin{array}{c}
i+1, i+2-2 p \\
2 i+2
\end{array} ; 1-t\right) .
$$

Since $\operatorname{Re}(2 p)>1$, Gauss' summation formula gives

$$
\lim _{t \rightarrow 0^{+}} t^{p-1} \check{h}_{i}(t)=\lim _{t \rightarrow 0^{+}} a_{i 2} F_{1}\left(\begin{array}{c}
i+1, i+2-2 p \\
2 i+2
\end{array} ; 1\right)=a_{i} \frac{(i+1)_{i+1}}{(2 p-1)_{i+1}} .
$$

In the case $2 p$, an integer $1 \leq 2 p \leq i+1$, we have

$$
\check{h}_{i}=a_{i} t^{p}(1-t)_{2}{ }_{2} F_{1}\left(\begin{array}{c}
i+1,2 p+i \\
2 i+2
\end{array} ; 1-t\right) .
$$

Therefore

$$
\lim _{t \rightarrow 0^{+}} t^{p-1} \check{h}_{i}(t)=\lim _{t \rightarrow 0^{+}} a_{i} t^{2 p-1}{ }_{2} F_{1}\left(\begin{array}{c}
i+1, i+2-2 p \\
2 i+2
\end{array} ; 1-t\right)=a_{i} \frac{(i+1)_{i+1}}{(2 p-1)_{i+1}}
$$

(see for example [1, Theorem 2.1.3]). This completes the proof of the proposition.

Proposition 5.15. Let $2 p=1$ and $H \in W_{\lambda},(\lambda=-1)$. Then

$$
\lim _{t \rightarrow 0^{+}} \frac{1}{t^{1 / 2} \log t} H(t)
$$

exists and is finite.

Proof. It is equivalent to prove that $\lim _{t \rightarrow 0^{+}} \frac{1}{t^{1 / 2} \log t} \check{H}(t)$ exists, if $\check{H}=U^{-1} H$. By Corollary 5.11(ii), for all $0 \leq i \leq \ell$ we have that

$$
\check{h}_{i}=a_{i} t^{1 / 2}(1-t)_{2}^{i} F_{1}\left(\begin{array}{c}
i+1, i+1 \\
2 i+2
\end{array} ; 1-t\right) .
$$

It is known that ([1, Theorem 2.1.3]):

$$
\lim _{t \rightarrow 0^{+}} \frac{{ }_{2} F_{1}\left(\begin{array}{c}
i+1, i+2-2 p \\
2 i+2
\end{array} ; 1-t\right)}{\log (1 / t)}=\frac{\Gamma(2 i+2)}{\Gamma(i+1) \Gamma(i+1)} .
$$

Therefore

$$
\lim _{t \rightarrow 0^{+}} \frac{1}{t^{1 / 2} \log t} \check{h}_{i}(t)=-a_{i} \frac{\Gamma(2 i+2)}{\Gamma(i+1) \Gamma(i+1)} .
$$

This completes the proof of the proposition. 


\section{Eigenfunctions of $D$ and $E$}

Here we exploit the decompositions of $V_{\lambda}, \lambda=4 p(p-1)$, established in Sec. 5:

(i) if $2 p \notin \mathbb{Z}, V_{\lambda}=V(p) \oplus V(1-p)=V(p) \oplus W_{\lambda}=V(1-p) \oplus W_{\lambda}$,

(ii) if $2 p \in \mathbb{Z}, 2 p \geq 1, V_{\lambda}=V(p) \oplus W_{\lambda}$,

(iii) if $2 p \in \mathbb{Z}, 2 p \leq 0, V_{\lambda}=V(1-p) \oplus W_{\lambda}$.

Proposition 6.1. For all $p \in \mathbb{C}$ the linear spaces $V_{\lambda}, W_{\lambda}$ and $V(p)$ are stable under the differential operator $E$. Therefore $E$ restricts to a linear map of each space into itself.

Proof. Since the differential operators $D$ and $E$ commute $E$ preserves the space $V_{\lambda}$.

If $H \in V(p)$ then $H=t^{p} F$, with $F$ analytic at $t=0$. Therefore $E H \in V(p)$ since

$$
\begin{aligned}
E H & =-4 t A_{0} H^{\prime}+\frac{4}{1-t} C_{0} H-\frac{4 t}{1-t} C_{1} H \\
& =t^{p}\left(-4 t A_{0} F^{\prime}-4 p A_{0} F+\frac{4}{1-t} C_{0} F-\frac{4 t}{1-t} C_{1} F\right) .
\end{aligned}
$$

In considering $W_{\lambda}$ we can assume that $\operatorname{Re}(2 p) \geq 1$. From Corollary 5.11 , we get that $H \in W_{\lambda}$ if and only if $H \in V_{\lambda}$ and $H$ is analytic at $t=1$. Thus to prove that $E H \in W_{\lambda}$ if $H \in W_{\lambda}$ we only have to see that $\frac{1}{1-t}\left(C_{0}-C_{1}\right) H$ is analytic at $t=1$, since we have

$$
\frac{4}{1-t} C_{0} H-\frac{4 t}{1-t} C_{1} H=4 C_{0}+\frac{4 t}{1-t}\left(C_{0}-C_{1}\right) H .
$$

Now from $D H=\lambda H$ and $H \in W_{\lambda}$ it follows that $\left(C_{0}+C_{1}\right) H(1)=0$. From Proposition 5.1, we get that an eigenvector of $C_{0}+C_{1}$ of eigenvalue zero is a scalar multiple of $u_{0}=(1, \ldots, 1)$. Then it is easy to check that $C_{0} u_{0}=C_{1} u_{0}=0$ and therefore $\frac{1}{1-t}\left(C_{0}-C_{1}\right) H$ is analytic at $t=1$.

The argument above shows that any eigenfunction $H$ of $D$ which has a finite limit $L$ as $t \rightarrow 1^{-}$has $L=c(1, \ldots, 1)$ for some scalar $c$.

Proposition 6.2. (i) Let $p \in \mathbb{C}, 2 p \notin \mathbb{Z}$ and let $\eta: V(p) \longrightarrow \mathbb{C}^{\ell+1}$ be the map defined by

$$
\eta: H \longmapsto \lim _{t \rightarrow 0^{+}}\left(t^{-p} H(t)\right) .
$$

Then $\eta$ is a linear isomorphism. Moreover the following is a commutative diagram

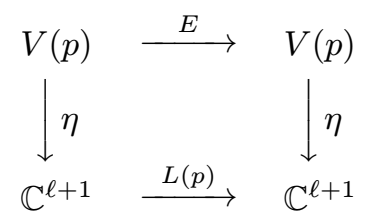


where $L(p)$ is the $(\ell+1) \times(\ell+1)$ matrix given by

$$
L(p)=4 C_{0}-4 p A_{0} .
$$

(ii) If $2 p \in \mathbb{Z}, 2 p \geq 1$, the same result holds.

(iii) If $2 p \in \mathbb{Z}, 2 p \leq 0$, the result holds by interchanging $p$ into $1-p$.

Proof. From Proposition 5.13, it follows that $\eta$ is well defined. Moreover it is clear that $\eta$ is a linear map between two vector spaces of dimension $\ell+1$. A function $H=t^{p} F(t) \in V(p)$, with $F(t)=\sum_{j=0}^{\infty} t^{j} F_{j}$ is given by solving the three term recursion relation (5.6):

$$
\begin{aligned}
& \left.j(j-1+2 p) F_{j}-\left(2 p(2 j+1)+2(j-1)^{2}-C_{0}-C_{1}\right)\right) F_{j-1} \\
& \quad+(j-1)(2 p+j-2) F_{j-2}=0 .
\end{aligned}
$$

This recurrence relation shows that the coefficient vector $F_{0}$ determines $H(t)$. Therefore $\eta$ is an injective map. The nonvanishing of the factor $j(j-1+2 p)$ is handled differently in cases (i), (ii) and (iii).

To prove the second assertion let $H(t)=t^{p} F \in V(p)$ with $F(t)=\sum_{j=0}^{\infty} t^{j} F_{j}$. Then by (6.1), we get

$$
\eta(E H)=\left(t^{-p}(E H)\right)(0)=-4 p A_{0} F_{0}+4 C_{0} F_{0}=L(p) F_{0}=L(p)(\eta(H)) .
$$

In Proposition 6.7, we shall prove that $L(p)$ is a diagonalizable matrix with eigenvalues

$$
\mu_{k}=\mu_{k}(p)=-4 p(\ell-2 k)-4 k(\ell-k+1), \quad \text { for } 0 \leq k \leq \ell,
$$

all with multiplicity one. Note that the sets of eigenvalues of $L(p)$ and $L(1-p)$ are the same, in fact we have

$$
\mu_{k}(1-p)=\mu_{\ell-k}(p), \quad \text { for all } 1 \leq k \leq \ell .
$$

The following theorem gives us all simultaneous solutions of the system $D H=$ $\lambda H$ and $E H=\mu H$, thus accomplishing task (i) at the beginning of Sec. 5 for $2 p \notin \mathbb{Z}$.

Theorem 6.3. Let $\lambda=4 p(p-1), 2 p \notin \mathbb{Z}$. A function $H=H(t), 0<t<1$ is a simultaneous eigenfunction of the system $D H=\lambda H$ and $E H=\mu H$ if and only if $H$ is of the following form:

$$
H(t)=\frac{t^{p}}{(1-t)^{\ell+1}} P(t)+\frac{t^{1-p}}{(1-t)^{\ell+1}} Q(t)
$$

where $P(t)=U \check{P}(t)$ and $Q(t)=U \check{Q}(t)$ and the ith-component of $\check{P}$ and $\check{Q}$ are

$$
\begin{gathered}
\check{P}_{i}(t)=\alpha_{i}(1-t)_{2}^{\ell-i} F_{1}\left(\begin{array}{c}
-i, 2 p-i-1 \\
2 p
\end{array} t\right), \\
\check{Q}_{i}(t)=\beta_{i}(1-t)_{2}^{\ell-i} F_{1}\left(\begin{array}{c}
-i,-2 p-i+1 \\
2-2 p
\end{array} t t\right)
\end{gathered}
$$

with $P(0)$ and $Q(0)$, respectively, eigenvectors of $L(p)$ and $L(1-p)$, of eigenvalue $\mu$. 
Proof. From Propositions 5.4 and 5.6, it follows that $H \in V_{\lambda}$ if and only if it is of the form stated in the first part of the theorem.

Since $V(p)$ and $V(1-p)$ are $E$-stable and $V_{\lambda}=V(p) \oplus V(1-p)$ it follows that $H \in V_{\lambda}$ is an eigenfunction of $E$ if and only if each summand of $H$ is an eigenfunction of $E$ with the same eigenvalue, which is equivalent by Proposition 6.2 to the fact that $P(0)$ and $Q(0)$ be, respectively, an eigenvector of $L(p)$ and $L(1-p)$ for the same eigenvalue.

We will see in Sec. 7 that even when $2 p \in \mathbb{Z}, H(t)$ allows a decomposition in the form above except for a limited range of values of $p$. Under the condition $2 p \notin \mathbb{Z}$ this decomposition is unique in the sense of Proposition 5.6. This uniqueness fails when $2 p \in \mathbb{Z}$.

To study the joint eigenfunctions $H=H(t)$ of the differential operators $D$ and $E$ when $2 p \in \mathbb{Z}$ we can use the decomposition of $V_{\lambda}$ given in Proposition 5.9.

The following three propositions describe the action of $E$ in $W_{\lambda}$ for any $p \in \mathbb{C}$.

Proposition 6.4. Let $p \in \mathbb{C}, \operatorname{Re}(2 p)>1$ and let $\eta: W_{\lambda} \longrightarrow \mathbb{C}^{\ell+1}, \lambda=4 p(p-1)$, be the map defined by

$$
\eta: H \longmapsto \lim _{t \rightarrow 0^{+}} t^{p-1} H(t) .
$$

Then $\eta$ is a linear isomorphism. Moreover the following is a commutative diagram

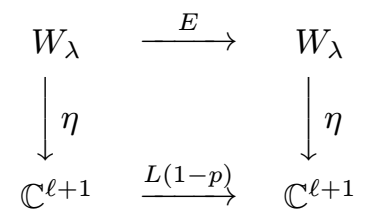

where $L(1-p)$ is the $(\ell+1) \times(\ell+1)$ matrix given by

$$
L(1-p)=4 C_{0}-4(1-p) A_{0} .
$$

A similar statement holds for $\operatorname{Re}(2 p)<1$, by changing $p$ into $1-p$.

Proof. From Proposition 5.14, it follows that $\eta$ is well defined. Moreover it is clear that $\eta$ is a linear map between two vector spaces of dimension $\ell+1$. So, we only have to verify that $\eta$ is injective.

Let $H \in W_{\lambda}$ and let $\check{H}=U^{-1} H=\left(\check{h}_{0}, \ldots, \check{h}_{\ell}\right)$. In the proof of Proposition 5.14, we have seen that in the case $2 p \notin \mathbb{Z}$ or $2 p$ an integer $0 \leq i<2 p-1$

$$
\check{h}_{i}=a_{i} t^{1-p}(1-t)_{2}^{i} F_{1}\left(\begin{array}{c}
i+1, i+2-2 p \\
2 i+2
\end{array} ; 1-t\right),
$$

and in the case $2 p$ an integer $2 p-1 \leq i \leq \ell$ we have

$$
\check{h}_{i}=a_{i} t^{p}(1-t)_{2}^{i} F_{1}\left(\begin{array}{c}
i+1,2 p+i \\
2 i+2
\end{array} ; 1-t\right) .
$$


We also proved that in both cases

$$
\lim _{t \rightarrow 0^{+}} t^{p-1} \check{h}_{i}(t)=a_{i} \frac{(i+1)_{i+1}}{(2 p-1)_{i+1}} .
$$

Now if $H \in W_{\lambda}$ satisfies $\eta(H)=0$ then $\lim _{t \rightarrow 0^{+}} t^{p-1} \breve{h}_{i}(t)=0$. So we have $a_{i}=0$ for all $0 \leq i \leq \ell$ and thus $H=0$. Therefore $\eta$ is an injective map.

To prove the second assertion let $H \in W_{\lambda}$. From (4.4) we have

$$
\eta(E H)=\lim _{t \rightarrow 0^{+}}\left(-4 A_{0} t^{p} H^{\prime}+\frac{4}{1-t} C_{0} t^{p-1} H-\frac{4}{1-t} C_{1} t^{p} H\right) .
$$

We first note that $\lim _{t \rightarrow 0^{+}} t^{p} H(t)=0$ because $\lim _{t \rightarrow 0^{+}} t^{p-1} H(t)$ exists and is finite. By L'Hospital rule we have

$$
\eta(H)=\lim _{t \rightarrow 0^{+}} \frac{t^{p} H(t)}{t}=p \eta(H)+\lim _{t \rightarrow 0^{+}} t^{p} H^{\prime}(t)
$$

thus $\lim _{t \rightarrow 0^{+}} t^{p} H^{\prime}(t)=(1-p) \eta(H)$. Therefore

$$
\eta(E H)=-4(1-p) A_{0} \eta(H)+4 C_{0} \eta(H)=L(1-p) \eta(H) .
$$

Proposition 6.5. Let $2 p=1$, that is $\lambda=-1$, and let $\eta: W_{\lambda} \longrightarrow \mathbb{C}^{\ell+1}$ be the map defined by

$$
\eta: H \longmapsto \lim _{t \rightarrow 0^{+}} \frac{1}{t^{1 / 2} \log t} H(t) .
$$

Then $\eta$ is a linear isomorphism. Moreover the following is a commutative diagram

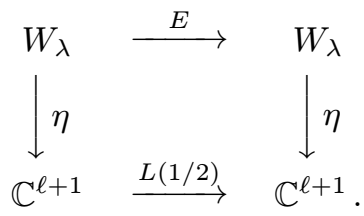

Proof. By Proposition 5.15, $\eta$ is a well defined linear map between two vector spaces of dimension $\ell+1$ (Corollary 5.12). Let $H \in W_{\lambda}$ be such that $\eta(H)=0$. From (5.15), we get $a_{i}=0$ for all $0 \leq i \leq \ell$. Thus we have that $H=0$, proving that $\eta$ is an isomorphism.

To prove that the diagram is commutative take $H \in W_{\lambda}$. By definition of the differential operator $E$ we have

$$
\eta(E H)=\lim _{t \rightarrow 0^{+}}\left(-\frac{4 t^{1 / 2}}{\log t} A_{0} H^{\prime}+\frac{4}{t^{1 / 2} \log t} C_{0} H-\frac{4 t^{1 / 2}}{\log t} C_{1} H\right) .
$$

We observe that

$$
\lim _{t \rightarrow 0^{+}} \frac{4}{t^{1 / 2} \log t} H=4 \eta(H)
$$

and that

$$
\lim _{t \rightarrow 0^{+}} \frac{4 t^{1 / 2}}{\log t} H=\lim _{t \rightarrow 0^{+}} \frac{4 t}{t^{1 / 2} \log t} H=0 .
$$


Finally by L'Hospital rule we have

$$
\eta(H)=\lim _{t \rightarrow 0^{+}} \frac{H(t)}{t^{1 / 2} \log t}=\lim _{t \rightarrow 0^{+}} \frac{H^{\prime}(t)}{\frac{1}{2} t^{-1 / 2} \log t+t^{-1 / 2}}=2 \lim _{t \rightarrow 0^{+}} \frac{t^{1 / 2} H^{\prime}(t)}{\log t} .
$$

Therefore $\eta(E H)=-2 A_{0} \eta(H)+4 C_{0} \eta(H)=L(1 / 2) \eta(H)$. This completes the proof of the proposition.

Proposition 6.6. Let $p \in \mathbb{C}, \operatorname{Re}(2 p)=1,2 p \neq 1$ and let $\eta: W_{\lambda} \longrightarrow \mathbb{C}^{\ell+1}$, $\lambda=4 p(p-1)$, be the map defined by

$$
\eta: H \longmapsto \lim _{t \rightarrow 0^{+}} t^{p-1} P(H)(t),
$$

where $P$ denotes the projection of $V_{\lambda}$ onto $V(1-p)$ along the subspace $V(p)$. Then $\eta$ is a linear isomorphism. Moreover the following is a commutative diagram

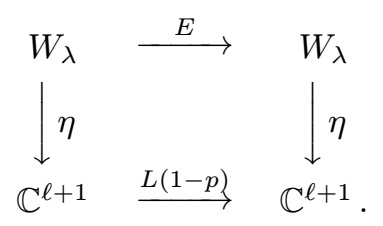

Proof. Let us observe that $\eta=\eta_{1} P$ where $\eta_{1}: V(1-p) \longrightarrow \mathbb{C}^{\ell+1}$ is the isomorphism introduced in Proposition 6.2, exchanging $p$ by $1-p$. Let $H \in W_{\lambda}$ such that $\eta(H)=0$. Then $P(H)=0$, i.e. $H \in V(p)$, but $V(p) \cap W_{\lambda}=0$. Hence $H=0$ proving that $\eta$ is an isomorphism since $\operatorname{dim} W_{\lambda}=\ell+1$ (see Corollary 5.12).

To prove the commutativity of the diagram we observe that given $H \in W_{\lambda}$ we have

$$
\eta(E H)=\eta_{1} P(E H)=\eta_{1} E P(H)=L(1-p) \eta_{1}(P(H))=L(1-p) \eta(H),
$$

by Propositions 6.1 and 6.2. This completes the proof of the proposition.

The construction above, with the introduction of the projection $P$ may appear different from that in Proposition 6.4 and 6.5. We explain now the need and the motivation behind this choice of $P$.

In this case $p=\frac{1}{2}+i \sigma, \sigma \neq 0$, real. Proposition 5.6 shows that any $H \neq 0$ in $W_{\lambda}$ has two components: one behaves like $t^{\frac{1}{2}+i \sigma}$ and the other one as $t^{\frac{1}{2}-i \sigma}$ as $t \rightarrow 0^{+}$. There is no way to multiply both components by a common function of $t$ to describe the asymptotic behaviour of $H$ at $t=0$. The only way out is to eliminate one of the two components first. This is the effect of the projection. In the cases considered in Propositions 6.4 and 6.5 this was not necessary, but we could have done it too.

This phenomenon is reminiscent of the presence of "Stokes lines" in the discussion of "dominant and recessive solutions" of an ordinary differential equation at an irregular singular point. 
Now is time to take the study of the matrix $L(p)$. In the case $\ell=0$ we have $L=E=0$ for all $p$, thus we assume below $\ell>0$.

Proposition 6.7. If $2 p \in \mathbb{C}$ is not an integer in the interval $-\ell+2 \leq 2 p \leq \ell$ then $L(p)=4 C_{0}-4 p A_{0}$ is diagonalizable with eigenvalues

$$
\mu_{k}=-4 p(\ell-2 k)-4 k(\ell-k+1), \quad \text { for } 0 \leq k \leq \ell,
$$

all with multiplicity one.

If $2 p$ is an integer in the interval $-\ell+2 \leq 2 p \leq \ell$ then $L(p)=4 C_{0}-4 p A_{0}$ is not diagonalizable. More precisely:

(i) If $1 \leq 2 p \leq \ell$ then $\mu_{k}, 0 \leq k<\frac{\ell+1-2 p}{2}$, are eigenvalues with multiplicity two, but with geometric multiplicity one; $\mu_{k}, \ell+1-2 p<k \leq \ell$ or $k=\frac{\ell+1-2 p}{2}$ (when $\ell+1-2 p$ is even), are eigenvalues with multiplicity one.

(ii) If $-\ell+2 \leq 2 p \leq 0$, then $\mu_{k}, 1-2 p \leq k<\frac{\ell+1-2 p}{2}$, are eigenvalues with multiplicity two, but with geometric multiplicity one; $\mu_{k}, 0 \leq k<1-2 p$ or $k=\frac{\ell+1-2 p}{2}$ (when $\ell+1-2 p$ is even), are eigenvalues with multiplicity one.

The eigenspace corresponding to the eigenvalue $\mu_{k}$ is generated by $v_{k}=$ $\left(v_{0, k}, \ldots, v_{\ell, k}\right)$, where $v_{i, k}=0$, for $0 \leq i<k, v_{k, k}=1$ and

$$
v_{k+j, k}=\prod_{i=1}^{j} \frac{(k+i)(\ell+1-i-k)}{i(\ell+1-2 p-2 k-i)},
$$

for $1 \leq j \leq \ell-k$. If $\mu_{k}$ is an eigenvalue with multiplicity two and $\mu_{k}=\mu_{k^{\prime}}$ we assume that $k>k^{\prime}$.

Proof. The matrix $L(p)=4 C_{0}-4 p A_{0}$ is a lower triangular matrix given by

$$
L(p)=\sum_{i=0}^{\ell} 4 i(\ell-i+1) E_{i, i-1}-4(i(\ell-i+1)+p(\ell-2 i)) E_{i, i} .
$$

Therefore the eigenvalues of $L(p)$ are $\mu_{k}=-4 p(\ell-2 k)-4 k(\ell-k+1)$, for each $k=0, \ldots, \ell$.

As a function of $k, \mu_{k}$ is symmetric around the point $\frac{\ell+1-2 p}{2}$, i.e. $\mu_{k}=$ $\mu_{\ell+1-2 p-k}$. Therefore there are eigenvalues with multiplicity two if and only if $2 p \in \mathbb{Z}$ and $0<\frac{\ell+1-2 p}{2}<\ell$, or equivalently $2 p \in \mathbb{Z}$ and $-\ell+2 \leq 2 p \leq \ell$. This proves the first assertion.

To prove (i) it is enough to visualize in the real line the points $0<\frac{\ell+1-2 p}{2}<$ $\ell+1-2 p<\ell$ and exploit their relative ordering and symmetry around $\frac{\ell+1-2 p}{2}$. Similarly to establish (ii) it is enough to visualize in the real line the points $0<$ $1-2 p<\frac{\ell+1-2 p}{2}<\ell$.

To prove the last assertion we first observe that $x=\left(x_{0}, \ldots, x_{\ell}\right)$ is an eigenvector of $L(p)$ corresponding to the eigenvalue $\mu_{k}$ if and only if the following equations hold

$$
4 i(\ell+1-i) x_{i-1}=\left(\mu_{k}-\mu_{i}\right) x_{i} \quad \text { for all } 1 \leq i \leq \ell .
$$


If $\mu_{k}$ is an eigenvalue with multiplicity two and $\mu_{k}=\mu_{k^{\prime}}$ we assume that $k>k^{\prime}$. Then from (6.5) it follows right away that $x_{i}=0$ for $0 \leq i \leq k-1$, and that $x_{k}$ determines $x_{i}$ for $k+1 \leq i \leq \ell$. Moreover a closer look at (6.5) shows that $v_{k}$, see (6.4), generates the eigenspace associated to the eigenvalue $\mu_{k}$.

By collecting our previous results we arrive at our punch line: for each $\ell \in \mathbb{Z}_{\geq 0}$ and $p \in \mathbb{C}$ there are exactly $\ell+1$ functions $H_{k}(t, p), 0 \leq k \leq \ell$, associated to spherical functions. Their explicit expressions are given in the next proposition. By the usual symmetry $p \mapsto 1-p$ we can assume $\operatorname{Re}(2 p) \geq 1$.

Theorem 6.8. Let $\lambda=4 p(p-1)$ with $\operatorname{Re}(2 p) \geq 1$. A function $H=H(t), 0<t<1$, corresponds to a spherical function $\Phi$ of type $\pi=\pi_{\ell}$ if and only if $H=U \check{H}$ where:

(i) If $2 p \notin \mathbb{Z}$,

$$
\check{h}_{i}=a_{i} t^{1-p}(1-t)_{2}^{i} F_{1}\left(\begin{array}{c}
i+1, i+2-2 p \\
2 i+2
\end{array} ; 1-t\right) \quad 0 \leq i \leq \ell .
$$

(ii) If $2 p \in \mathbb{Z}$,

$$
\check{h}_{i}= \begin{cases}a_{i} t^{1-p}(1-t)_{2}{ }_{2} F_{1}\left(\begin{array}{c}
i+1, i+2-2 p \\
2 i+2
\end{array} ; 1-t\right) & 0 \leq i \leq 2 p-2, \\
a_{i} t^{p}(1-t)^{i}{ }_{2} F_{1}\left(\begin{array}{c}
i+1,2 p+i \\
2 i+2
\end{array} ; 1-t\right) & 2 p-1 \leq i \leq \ell .\end{cases}
$$

When $2 p \notin \mathbb{Z}$ or $i=0, \ldots, 2 p-2, a_{i}=\alpha_{i} \frac{(2 p-1)_{i+1}}{(i+1)_{i+1}}$ and when $i=2 p-1, \ldots, \ell$, $a_{i}=\alpha_{i} \frac{(1-2 p)_{i+1}}{(i+1)_{i+1}}$. In both cases $\alpha_{0}, \ldots, \alpha_{\ell}$ is such that $U\left(\alpha_{0}, \ldots, \alpha_{\ell}\right)^{T}$ is an eigenvector of the matrix $L(1-p)$ of eigenvalue $\mu_{k}, 0 \leq k \leq \ell$, and $a_{0}=1$.

Moreover $\Omega \Phi=\left(\frac{\lambda}{4}+\mu_{k}+\ell(\ell+2)\right) \Phi$ and $\bar{\Omega} \Phi=\frac{\lambda}{4} \Phi$.

The functions $H_{k}(t, p)$ resulting from the $\ell+1$ choices of $\mu_{k}$ above will be the main characters in Secs. 7 and 9. We refer to these functions as the families associated to a given $\ell$.

In the following proposition we clarify the correspondence $(p, k) \mapsto \Phi_{(p, k)}$ which assigns to the pair $(p, k) \in \mathbb{C} \times \mathbb{Z}, 0 \leq k \leq \ell$, the spherical function $\Phi_{(p, k)}$ of type $\ell$ associated to the eigenvalues $\mu_{k}=-4 p(\ell-2 k)-4 k(\ell-k+1)$ and $\lambda=4 p(p-1)$.

Proposition 6.9. If $\Phi$ is a spherical function on $G$ of type $\ell$ then $\Phi=\Phi_{(p, k)}$ for some pair $(p, k)$. Moreover $\Phi_{(p, k)}=\Phi_{\left(p^{\prime}, k^{\prime}\right)}$ if and only if

$$
\left(p^{\prime}, k^{\prime}\right)= \begin{cases}(p, k), & \\ (1-p, \ell-k), & \text { if } \ell+1-k-2 p \in\{0, \ldots, \ell\}, \\ (p, \ell+1-k-2 p) & \text { if } k-1+2 p \in\{0, \ldots, \ell\} .\end{cases}
$$


Proof. Let $\lambda(p, k)=4 p(p-1)$ and $\mu(p, k)=-4 p(\ell-2 k)-4 k(\ell-k+1)$. By Proposition 6.10 below the problem reduces to finding the pairs $\left(p^{\prime}, k^{\prime}\right)$ such that $\lambda(p, k)=\lambda\left(p^{\prime}, k^{\prime}\right)$ and $\mu(p, k)=\mu\left(p^{\prime}, k^{\prime}\right)$.

Now $\lambda(p, k)=\lambda\left(p^{\prime}, k^{\prime}\right)$ if and only if $\left(p-p^{\prime}\right)\left(p+p^{\prime}-1\right)=0$, that is, if and only if $p^{\prime}=p$ or $p^{\prime}=1-p$.

Also it is easy to check that $\mu(p, k)=\mu\left(p, k^{\prime}\right)$ if and only if $k=k^{\prime}$ or $k^{\prime}=$ $\ell+1-k-2 p$, and that $\mu(p, k)=\mu(1-p, \ell-k)$.

Therefore, for $\left(p^{\prime}, k^{\prime}\right)$ we have the following possibilities: $(p, k)$ and $(p, \ell+1-$ $k-2 p)$, or $(1-p, \ell-k)$ and $(1-p, \ell+1-(\ell-k)-2(1-p))=(1-p, k-1+2 p)$. This completes the proof of the proposition.

Throughout this paper we have carried out a bare hands construction of the spherical functions starting from their definition. The rest of this section relates such a construction to the representation theory of $G$.

Spherical functions of type $\delta \in \hat{K}$ arise in a natural way upon considering representations of $G$. If $g \mapsto U(g)$ is a continuous representation of $G$, say on a complete, locally convex, Hausdorff topological vector space $E$, then

$$
P(\delta)=\int_{K} \chi_{\delta}\left(k^{-1}\right) U(k) d k
$$

is a continuous projection of $E$ onto $P(\delta) E=E(\delta) ; E(\delta)$ consists of those vectors in $E$, the linear span of whose $K$-orbit is finite dimensional and splits into irreducible $K$-subrepresentations of type $\delta$. Whenever $E(\delta)$ is finite dimensional and not zero, the function $\Phi_{\delta}: G \longrightarrow \operatorname{End}(E(\delta))$ defined by $\Phi_{\delta}(g) a=P(\delta) U(g) a, g \in G, a \in$ $E(\delta)$ is a spherical function of type $\delta$. Moreover any irreducible spherical function arises in this way from a topologically irreducible representation of $G$, (see $[6,13]$ ).

If a spherical function $\Phi$ is associated to a Banach representation of $G$ then it is quasi-bounded, in the sense that there exists a semi-norm $\rho$ on $G$ and $M \in \mathbb{R}$ such that $\|\Phi(g)\| \leq M \rho(g)$ for all $g \in G$. Conversely, if $\Phi$ is an irreducible quasibounded spherical function on $G$, then it is associated to a topologically irreducible Banach representation of $G$ (see $[6,13])$. Thus if $G$ is compact any irreducible spherical function on $G$ is associated to a Banach representation of $G$, which is finite dimensional by the Peter-Weyl theorem.

When $G$ is a connected Lie group any spherical function is analytic, hence it is determined by $(D \Phi)(e)$ for all left invariant differential operators $D$ on $G$. We also need to quote the following fact (cf. [13, Remark 4.7]).

Proposition 6.10. Let $\Phi, \Psi: G \longrightarrow \operatorname{End}(V)$ be two spherical functions on a connected Lie group $G$ of the same type $\delta \in K$. Then $\Phi=\Psi$ if and only if $(D \Phi)(e)=$ $(D \Psi)(e)$ for all $D \in D(G)^{K}$.

If $G$ is a noncompact connected semisimple Lie group with finite center, and $K$ is a maximal compact subgroup of $G$, then, from Casselman's subrepresentation theorem (see [11 p. 238]), we know that any irreducible $(\mathfrak{g}, K)$ module 
can be realized inside a generalized (also known as nonunitary) principal series representation of $G$. Thus, in particular, any irreducible spherical function of $(G, K)$ is associated to a generalized principal series representation of $G$, and thus it is quasi-bounded.

This can be seen directly for $(G, K)=(\mathrm{SL}(2, \mathbb{C}), \mathrm{SU}(2))$ from our explicit construction of all irreducible spherical functions. Let $M A N$ be the upper triangular subgroup of $G$. Define $\sigma$ on $M$ and $\nu$ on $\mathfrak{a}$ by

$$
\begin{aligned}
\sigma\left(\begin{array}{cc}
e^{i \theta} & 0 \\
0 & e^{-i \theta}
\end{array}\right) & =e^{i r \theta}, \\
\nu\left(\begin{array}{cc}
t & 0 \\
0 & -t
\end{array}\right) & =i v t,
\end{aligned}
$$

for $r \in \mathbb{Z}$ and $v \in \mathbb{C}$. We also point out that the half-sum of the positive restricted roots is given by

$$
\rho\left(\begin{array}{cc}
t & 0 \\
0 & -t
\end{array}\right)=2 t .
$$

Then $\operatorname{man} \mapsto e^{\nu \log a} \sigma(m)$ is a representation of $M A N$, and it is this representation that we induce to $G$ to construct its generalized principal series representation. Thus we put $U^{\sigma, \nu}=\operatorname{Ind} d_{M A N}^{G}$.

A dense subspace of the representation space of $U=U^{\sigma, \nu}$ is

$$
\left\{F: G \longrightarrow \mathbb{C} \text { continuous }: F(x m a n)=e^{-(\nu+\rho) \log a} \sigma(m)^{-1} F(x)\right\}
$$

with norm

$$
\|F\|^{2}=\int_{K}|F(k)|^{2} d k
$$

and $G$ acts by

$$
U^{\sigma, \nu}(g) F(x)=F\left(g^{-1} x\right) .
$$

The actual Hilbert space and representation are then obtain by completion.

A $K$ type $\pi_{\ell}$ occurs in $U^{\sigma, \nu}$ if and only if $r=\ell-2 j$ for some $0 \leq j \leq \ell$ (Frobenius Reciprocity Theorem). If this is the case, let $\Phi=\Phi_{v, r}$ be the spherical function (irreducible) of type $\pi_{\ell}$ associated to $U^{\sigma, \nu}$, and let $P_{\ell}$ be the corresponding projection onto the $K$-isotypic component. To identify $\Phi_{v, r}$ we just need to compute $\left[\Omega \Phi_{v, r}\right](e)$ and $\left[\bar{\Omega} \Phi_{v, r}\right](e)$, (Proposition 6.10). We start by observing that

$$
\begin{aligned}
& 4 \Omega=H_{1}^{2}-H_{2}^{2}-4 H_{1}+4 i H_{2}-2 i H_{1} H_{2}+4 X_{1} Y_{1}-4 X_{2} Y_{2}-4 i X_{1} Y_{2}-4 i X_{2} Y_{1}, \\
& 4 \bar{\Omega}=H_{1}^{2}-H_{2}^{2}-4 H_{1}-4 i H_{2}+2 i H_{1} H_{2}+4 X_{1} Y_{1}-4 X_{2} Y_{2}+4 i X_{1} Y_{2}+4 i X_{2} Y_{1},
\end{aligned}
$$

where $X_{1}=\frac{1}{2}\left(V_{1}+W_{1}\right), Y_{1}=\frac{1}{2}\left(V_{1}-W_{1}\right), X_{2}=\frac{1}{2}\left(W_{2}+V_{2}\right)$ and $Y_{2}=\frac{1}{2}\left(W_{2}-V_{2}\right)$.

If $X \in \mathfrak{n}, Y \in \mathfrak{g}$, and $F$ is a smooth function in the induced space, then

$$
\dot{U}(X) \dot{U}(Y) F(e)=\left.\frac{d}{d t} \dot{U}(Y) F\left((\exp t X)^{-1}\right)\right|_{t=0}=0,
$$


because $\dot{U}(Y) F$ is right invariant under $N$. On the other hand if $X=H+T \in \mathfrak{a}+\mathfrak{m}$, then

$$
\begin{aligned}
\dot{U}(X) F(e) & =\left.\frac{d}{d t} \dot{U} F\left((\exp t X)^{-1}\right)\right|_{t=0}=\left.\frac{d}{d t}\left(e^{\nu+\rho}(\exp t H) \sigma(\exp t T)\right) F(e)\right|_{t=0} \\
& =((\nu+\rho)(H)+\dot{\sigma}(T)) F(e) .
\end{aligned}
$$

Since $X_{1}, X_{2} \in \mathfrak{n}, H_{1} \in \mathfrak{a}$ and $H_{2} \in \mathfrak{m}$, we obtain

$$
\begin{aligned}
4 \dot{U}(\Omega) F(e) & =\left((i v+2)^{2}-(i r)^{2}-4(i v+2)+4 i(i r)-2 i(i v+2)(i r)\right) F(e) \\
& =(i v+2+r)(i v-2+r) F(e), \\
4 \dot{U}(\bar{\Omega}) F(e) & =\left((i v+2)^{2}-(i r)^{2}-4(i v+2)-4 i(i r)+2 i(i v+2)(i r)\right) F(e) \\
& =(i v+2-r)(i v-2-r) F(e) .
\end{aligned}
$$

Since $\Omega, \bar{\Omega} \in D(G)^{G}, \dot{U}(\Omega)$ and $\dot{U}(\bar{\Omega})$ commute with $U(g)$ for all $g \in G$. Therefore $\dot{U}(\Omega)=(i v+2+r)(i v-2+r) I$ and $\dot{U}(\bar{\Omega})=(i v+2-r)(i v-2-r) I$.

Now $\Omega \Phi(g) P_{\ell}=P_{\ell} U(g) \dot{U}(\Omega) P_{\ell}=4(i v+2+r)(i v-2+r) \Phi(g) P_{\ell}$. A similar argument holds for $\bar{\Omega}$. Thus

$$
\Omega \Phi=4(i v+2+r)(i v-2+r) \Phi, \quad \text { and } \quad \bar{\Omega} \Phi=4(i v+2-r)(i v-2-r) \Phi .
$$

Proposition 6.11. For $v \in \mathbb{C}$ and $r=\ell-2 j, 0 \leq j \leq \ell$, let $p=\frac{1}{4}(i v+2-r)$ and $k=\frac{1}{2}(r+\ell)$. Then the spherical function $\Phi_{v, r}$ of type $\pi_{\ell}$ associated to $U^{\sigma, \nu}$ is equivalent to $\Phi_{(p, k)}$.

Proof. As we said before, the only thing we have to verify is that the eigenvalues of $\Omega$ and $\bar{\Omega}$ corresponding to $\Phi_{v, r}$ and $\Phi_{(p, k)}$ are respectively the same. Taking into account Remark 4.9, Theorem 6.8 and (6.3), this in turn amounts to checking that

$$
(i v+2-r)(i v-2-r)=4 p(4 p-4)
$$

and

$$
i v r=-4 p(\ell-2 k)-4 k(\ell-k+1)+\ell(\ell+2),
$$

which is straightforward.

Remark 6.12. The resulting relations $v=i(\ell-2 k+2-4 p), r=2 k-\ell$, with $p \in \mathbb{C}$ and $0 \leq k \leq \ell$ show that the $\operatorname{map}(v, r) \mapsto(p, k)$ is one to one and onto. Thus any irreducible spherical function is equivalent to one associated to a principal series representation as advertised above. Moreover the map $(v, r) \mapsto(-v,-r)$ corresponds to the map $(p, k) \mapsto(1-p, \ell-k)$. This explains the equivalence between $\Phi_{(p, k)}$ and $\Phi_{(1-p, \ell-k)}$ (Proposition 6.9) in terms of the equivalence between $U^{\sigma, \nu}$ and $U^{w \cdot \sigma, w \cdot \nu}$, where $w$ is the nontrivial element of the restricted Weyl group $W(\mathfrak{g}, \mathfrak{a})$. 


\section{Some Examples and a Conjecture}

The results in Secs. 5 and 6 include a number of different expressions for the components of the vector $H(t)$ corresponding to a spherical function. Some of these expressions are rather elaborate; for instance those given in Theorem 6.8 involve Gauss' hypergeometric functions as well as Hahn polynomials. Others involve simple linear combinations of $t^{p}$ and $t^{1-p}$ with rational coefficients, as in Theorem 6.3. This "embarrassment of riches" results from different decompositions of $V_{\lambda}$ as a direct sum of subspaces. These decompositions are dictated by the nature of $p$ as summarized at the beginning of Sec. 6 . The case $2 p \notin \mathbb{Z}$ is simpler to deal with and this is reflected in the simpler expressions appearing in Theorem 6.3.

The relative merit of these or other explicit representations of $H(t)$ depends on the task at hand. In this section we identify those cases when $H(t)$ contains logarithmic terms. For this task an expression as in Theorem 6.3 is most convenient and we show that its validity goes beyond the condition $2 p \notin \mathbb{Z}$.

The first part of this section is done entirely in terms of explicit examples given in full detail. This should allow the reader to check many points of the theoretical treatment in the rest of the paper.

We close with a conjecture of the form of $H(t)$ which is a "first cousin" of a conjecture in [7].

The case $\ell=0$. In the case $\ell=0$, there is only one equation, namely

$$
4 t^{2} h^{\prime \prime}(t)-\frac{8 t^{2}}{1-t} h^{\prime}(t)=4 p(p-1) h(t)
$$

with a solution

$$
h(t)=\frac{t^{p}-t^{1-p}}{(2 p-1)(t-1)}
$$

as in Theorem 6.3. This expression is valid as soon as $p$ is not $\frac{1}{2}$. In the case $p=\frac{1}{2}$ the spherical function is given by taking the limit in the expression above. One gets

$$
h(t)=\frac{\sqrt{t} \log (t)}{t-1} .
$$

This, as well as the examples below, illustrates the fact that the result in Theorem 6.3 is valid as long as $2 p$ is not an integer in the range $-\ell+1,-\ell+$ $2, \ldots,-1,0,1, \ldots, \ell+1$. In fact it is only for these values of $p$ that spherical functions will contain a logarithmic term.

We display this in the cases $\ell=1$ and $\ell=2$ below. Afterwards we describe the pattern in the case of $\ell=5$.

The case $\ell=1$. We have one family with $\mu=-4 p$ (corresponding to $k=0$ ).

$$
h_{0}(t)=\frac{t^{2-p}}{(p-1)(2 p-1)(t-1)^{2}}+\frac{t^{p}(2(p-1) t-2 p+1)}{(p-1)(2 p-1)(t-1)^{2}},
$$




$$
h_{1}(t)=\frac{t^{p}}{(p-1)(2 p-1)(t-1)^{2}}-\frac{t^{1-p}((2 p-1) t-2 p+2)}{(p-1)(2 p-1)(t-1)^{2}} .
$$

The other family (corresponding to $k=1$ ) is obtained by exchanging $p$ and $1-p$.

We have an exceptional form for $p=\frac{1}{2}$, namely

$$
h_{0}(t)=\frac{2 \sqrt{t}(t \log (t)-t+1)}{(t-1)^{2}}, \quad h_{1}(t)=-\frac{2 \sqrt{t}(\log (t)-t+1)}{(t-1)^{2}}
$$

and for $p=1$, where we get

$$
h_{0}(t)=-\frac{2 t(\log (t)-t+1)}{(t-1)^{2}}, \quad h_{1}(t)=\frac{2(t \log (t)-t+1)}{(t-1)^{2}} .
$$

As usual (for reasons of symmetry) it is enough to consider $p$ to one side of the value $\frac{1}{2}$.

In summary we have, for $\ell=1$, a total of two spherical functions with logarithmic terms.

The case $\ell=2$. We have three families corresponding to $k=0,1,2$.

The first one has $\mu=-8 p$ and the vectors $P(t)$ and $Q(t)$ in Theorem 6.3 can be taken to be

$$
\begin{aligned}
& P_{0}(t)=\frac{3\left(2 p^{2} t^{2}-5 p t^{2}+3 t^{2}-4 p^{2} t+8 p t-3 t+2 p^{2}-3 p+1\right)}{(p-1)(2 p-3)(2 p-1)}, \\
& P_{1}(t)=\frac{3(2 p t-3 t-2 p+1)}{(p-1)(2 p-3)(2 p-1)}, \\
& P_{2}(t)=\frac{3}{(p-1)(2 p-3)(2 p-1)},
\end{aligned}
$$

and

$$
\begin{aligned}
Q_{0}(t) & =-\frac{3 t^{2}}{(p-1)(2 p-3)(2 p-1)}, \\
Q_{1}(t) & =\frac{3 t(2 p t-t-2 p+3)}{(p-1)(2 p-3)(2 p-1)}, \\
Q_{2}(t) & =-\frac{3\left(2 p^{2} t^{2}-3 p t^{2}+t^{2}-4 p^{2} t+8 p t-3 t+2 p^{2}-5 p+3\right)}{(p-1)(2 p-3)(2 p-1)},
\end{aligned}
$$

as long as $p \neq \frac{1}{2}, 1, \frac{3}{2}$.

The second family of solutions (well defined for $p$ different from $0, \frac{1}{2}, 1$ has $\mu=-8$. The vector $P(t)$ can be taken as

$$
P_{0}(t)=\frac{3 t(p t-t-p)}{(p-1) p(2 p-1)}
$$




$$
\begin{aligned}
& P_{1}(t)=\frac{3\left(p^{2} t^{2}-2 p t^{2}+t^{2}-2 p^{2} t+2 p t+t+p^{2}\right)}{(p-1) p(2 p-1)}, \\
& P_{2}(t)=\frac{3(p t-t-p)}{(p-1) p(2 p-1)}
\end{aligned}
$$

and the vector $Q(t)$ can be taken as

$$
\begin{aligned}
Q_{0}(t) & =\frac{3 t(p t-p+1)}{(p-1) p(2 p-1)}, \\
Q_{1}(t) & =-\frac{3\left(p^{2} t^{2}-2 p^{2} t+2 p t+t+p^{2}-2 p+1\right)}{(p-1) p(2 p-1)}, \\
Q_{2}(t) & =\frac{3(p t-p+1)}{(p-1) p(2 p-1)} .
\end{aligned}
$$

By changing $p$ into $1-p$ in the first family we get the third family of spherical functions, well defined for $p$ not in the set $-\frac{1}{2}, 0, \frac{1}{2}$. We have $\mu=8 p-8$, corresponding to $k=2$. The vector $P(t)$ can be taken as

$$
\begin{aligned}
& P_{0}(t)=\frac{3 t^{2}}{p(2 p-1)(2 p+1)} \\
& P_{1}(t)=\frac{3 t(2 p t-t-2 p-1)}{p(2 p-1)(2 p+1)} \\
& P_{2}(t)=\frac{3\left(2 p^{2} t^{2}-p t^{2}-4 p^{2} t+t+2 p^{2}+p\right)}{p(2 p-1)(2 p+1)},
\end{aligned}
$$

and the vector $Q(t)$ can be taken as

$$
\begin{aligned}
& Q_{0}(t)=-\frac{3\left(2 p^{2} t^{2}+p t^{2}+4 p^{2} t+t+2 p^{2}-p\right)}{p(2 p-1)(2 p+1)}, \\
& Q_{1}(t)=\frac{3(2 p t+t-2 p+1)}{p(2 p-1)(2 p+1)}, \\
& Q_{2}(t)=-\frac{3}{p(2 p-1)(2 p+1)} .
\end{aligned}
$$

Now we analyze each one of the exceptional points.

Exceptional point $p=-\frac{1}{2}$. The third family gives

$$
\begin{aligned}
& h_{0}(t)=\frac{3 t^{3 / 2}\left(2 \log (t)+t^{2}-4 t+3\right)}{2(t-1)^{3}}, \\
& h_{1}(t)=-\frac{3 t^{1 / 2}\left(2 t \log (t)-t^{2}+1\right)}{(t-1)^{3}}, \\
& h_{2}(t)=\frac{3 t^{-1 / 2}\left(2 t^{2} \log (t)-3 t^{2}+4 t-1\right)}{2(t-1)^{3}} .
\end{aligned}
$$


Exceptional point $p=0$. The third family gives

$$
\begin{aligned}
& h_{0}(t)=-\frac{3 t\left(2 t \log (t)-t^{2}+1\right)}{(t-1)^{3}}, \\
& h_{1}(t)=\frac{6 t(t \log (t)+\log (t)-2 t+2)}{(t-1)^{3}}, \\
& h_{2}(t)=-\frac{3\left(2 t \log (t)-t^{2}+1\right)}{(t-1)^{3}} .
\end{aligned}
$$

The second family gives the same spherical function, since in both cases we have $\lambda=0, \mu=-8$.

Exceptional point $p=\frac{1}{2}$. The third family gives

$$
\begin{aligned}
& h_{0}(t)=\frac{3 t^{1 / 2}\left(2 t^{2} \log (t)-3 t^{2}+4 t-1\right)}{2(t-1)^{3}}, \\
& h_{1}(t)=-\frac{3 t^{1 / 2}\left(2 t \log (t)-t^{2}+1\right)}{(t-1)^{3}}, \\
& h_{2}(t)=\frac{3 t^{1 / 2}\left(2 \log (t)+t^{2}-4 t+3\right)}{2(t-1)^{3}} .
\end{aligned}
$$

The second family gives

$$
\begin{aligned}
& h_{0}(t)=\frac{6 t^{3 / 2}(t \log (t)+\log (t)-2 t+2)}{(t-1)^{3}}, \\
& h_{1}(t)=-\frac{3 t^{1 / 2}\left(t^{2} \log (t)+6 t \log (t)+\log (t)-4 t^{2}+4\right)}{(t-1)^{3}}, \\
& h_{2}(t)=\frac{6 t^{1 / 2}(t \log (t)+\log (t)-2 t+2)}{(t-1)^{3}} .
\end{aligned}
$$

The first family gives the same spherical function as the one obtained from the third family, since they share the value of $(\lambda, \mu)$.

Here we find something different. The first and third family give (unsurprisingly) the same limiting spherical function, but the second family gives something else. This is as it should be since the values of $(\lambda, \mu)$ for the first and third families are $\lambda=-1, \mu=-4$ while for the second family we get $\lambda=-1, \mu=-8$.

The exceptional points $p=1$ and $p=\frac{3}{2}$ reproduce the results of the points $p=0$ and $p=-\frac{1}{2}$, respectively.

In summary, when $\ell=2$, we have a total of four spherical functions with logarithmic terms, one each from the pairs $p=-\frac{1}{2}, \frac{3}{2}$ and $p=0,1$ and two from the value $p=\frac{1}{2}$.

In Fig. 1, we display the three families in the $(p, \mu)$-plane. The exceptional points are marked on the $p$-axis. On each one of the lines giving the different families we mark the points giving rise to spherical functions with logarithmic terms. 


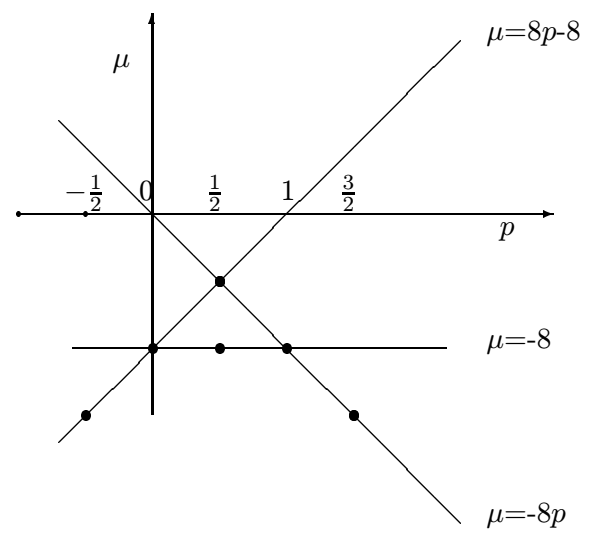

Fig. 1.

The case $\ell=5$. It is now clear what the general pattern is, as illustrated here for $\ell=5$.

There are six families of spherical functions and each one of them has six exceptional values of $p$. The table below gives the exceptional values of $p$ for each family.

$$
\begin{array}{ccccccc}
1 \text { st } & -2, & -\frac{3}{2}, & -1, & -\frac{1}{2}, & 0, & \frac{1}{2} \\
2 n d & -\frac{3}{2}, & -1, & -\frac{1}{2}, & 0, & \frac{1}{2}, & 1 \\
3 r d & -1, & -\frac{1}{2}, & 0, & \frac{1}{2}, & 1, & \frac{3}{2} \\
4 t h & -\frac{1}{2}, & 0, & \frac{1}{2}, & 1, & \frac{3}{2}, & 2 \\
5 t h & 0, & \frac{1}{2}, & 1, & \frac{3}{2}, & 2, & \frac{5}{2} \\
6 t h & \frac{1}{2}, & 1, & \frac{3}{2}, & 2, & \frac{5}{2}, & 3
\end{array}
$$

and the corresponding values of $\mu$ are listed below

$$
\mu=-20 p,-4(3 p+5),-4(p+8), 4(p-9), 4(3 p-8), 20(p-1) .
$$

The first and sixth family are related by the exchange of $p$ into $1-p$, and the same relation connects the second and fifth families, and the third and fourth ones. This relation is clearly manifested in the corresponding exceptional sets as well as in the values of $\mu$ above.

For $p=-2$ (and the corresponding symmetric value $p=3$ ) the first and sixth families give one spherical function with $\lambda=24, \mu=-60$.

For $p=-\frac{3}{2}$ (and its companion $p=\frac{5}{2}$ ) the first and sixth families contribute one spherical function, with $(\lambda, \mu)=(15,-50)$. The second and fifth families give 
a common spherical function with $(\lambda, \mu)=(15,-50)$. In this way we get one more spherical function with logarithmic terms.

For $p=-1$ (and its companion value $p=2$ ) the first and sixth families as well as the third and fourth families give a common spherical function with $(\lambda, \mu)=$ $(8,20)$. The second and fifth families give a common spherical function with $(\lambda, \mu)=$ $(8,-44)$. This gives us two more spherical functions with logarithmic terms.

For $p=-\frac{1}{2}$ (and its companion value $p=\frac{3}{2}$ ) the first and sixth families give a spherical function which is common with the one that the third family gives for $p=3 / 2$ and the fourth gives for $p=-\frac{1}{2}$. The values of $(\lambda, \mu)$ are $(3,-30)$. Now $p=-\frac{1}{2}$ is also an exceptional value for the third family and $p=\frac{3}{2}$ is one for the fourth family. This produces another spherical function which coincides with the one that corresponds to the second and fifth family. The value of $(\lambda, \mu)$ is $(3,-38)$. So, for the pair $p=-\frac{1}{2}, \frac{3}{2}$ there are two spherical functions with logarithmic terms.

For the pair $p=0,1$, there are three different spherical functions corresponding to the first and sixth families, the second and fifth, and the third and fourth. The corresponding values for the eigenvalues of $D$ and $E$ are $(0,-20),(0,-32)$ and $(0,-36)$.

For $p=\frac{1}{2}$, there are three different spherical functions corresponding to the first and sixth families, the second and fifth families, and the third and fourth ones. The values for the parameters $(\lambda, \mu)$ are $(-1,-10),(-1,-26)$ and $(-1,-34)$, respectively.

In summary, for $\ell=5$, we have a total of twelve spherical functions with logarithmic terms.

We close the section by stating the conjecture advertised above. From Theorem 6.8, we get a completely explicit form for the spherical functions in terms of Gauss' hypergeometric functions and a matrix $U$ whose columns are given by Hahn polynomials (Proposition 5.1). This gives our functions $H(t)$ as a linear combination of the classical functions.

A reader familiar with the results in [7] may wonder if it is possible to give an expression for $H(t)$ in terms of a single generalized hypergeometric function. Such a result is offered below in the form of a conjecture.

Conjecture 7.1. For a given $\ell \geq 0$, the functions $H(t)$ have components that are expressed in terms of a special class of generalized hypergeometric functions of the form ${ }_{p+2} F_{p+1}$, namely

$$
{ }_{p+2} F_{p+1}\left(\begin{array}{c}
a, b, s_{1}+1, \ldots, s_{p}+1 \\
c, s_{1}, s_{2}, \ldots, s_{p}
\end{array} ;\right) .
$$

More explicitly, the $k$ th family of spherical functions, characterized by $\lambda=$ $4 p(p-1), \mu_{k}=-4 p(\ell-2 k)-4 k(\ell-k+1)$, has components $h_{i}(t)$ given by

$$
h_{i}(t)=t_{a(\ell, i, k)}^{p+k-i} F_{a(\ell, i, k)-1}\left(\begin{array}{c}
1,2 p+k-i,\left\{s_{j}+1\right\} \\
\ell+2,\left\{s_{j}\right\}
\end{array} ; 1-t\right)
$$


with $a(\ell, i, k)=\min \{i+k+2, \ell+2\}$. Here $\left\{s_{j}\right\}$ denotes a row vector of $a(\ell, i, k)-2$ denominator parameters and $\left\{s_{j}+1\right\}$ are the same coefficients shifted up by one.

\section{Unitary Spherical Functions}

Given a function $\Phi: G \longrightarrow \operatorname{End}(V)$, where $V$ is a finite dimensional complex vector space with an inner product, we define $\check{\Phi}: G \longrightarrow \operatorname{End}(V)$ by $\check{\Phi}(g)=\Phi\left(g^{-1}\right)^{*}$, where * denotes the operation of taking adjoint.

Proposition 8.1. The function $\Phi$ is spherical of type $\delta \in \hat{K}$ if and only if $\check{\Phi}$ is spherical of type $\delta$.

Proof. Let us assume that $\Phi$ is spherical of type $\delta$. Then

$$
\begin{aligned}
\check{\Phi}(x) \check{\Phi}(y) & =\left(\Phi\left(y^{-1}\right) \Phi\left(x^{-1}\right)\right)^{*}=\left(\int_{K} \chi_{\delta}\left(k^{-1}\right) \Phi\left(y^{-1} k x^{-1}\right) d k\right)^{*} \\
& =\int_{K} \chi_{\delta}(k) \check{\Phi}\left(x k^{-1} y\right) d k .
\end{aligned}
$$

Moreover $\check{\Phi}(e)=\Phi(e)^{*}=I$, hence $\check{\Phi}$ is a spherical function of type $\delta$. On the other hand since $\check{\Phi}=\Phi$ the proposition follows.

Definition 8.2. A spherical function $\Phi: G \longrightarrow \operatorname{End}(V)$ is said to be unitarizable if there exists an inner product on $V$ such that $\Phi(g)^{*}=\Phi\left(g^{-1}\right)$, for all $g \in G$. In such a case we also say that $\Phi$ is a unitary spherical function. In other words $\Phi$ is unitary if and only if $\check{\Phi}=\Phi$.

Proposition 8.3. If $\Phi: G \longrightarrow \operatorname{End}(V)$ is a unitarizable irreducible spherical function then there exists a unique inner product on $V$, up to a positive multiplicative constant, which turns $\Phi$ unitary.

Proof. Let $($,$) and \langle$,$\rangle be two inner products on V$ which make $\Phi$ unitary. Then there exists a linear operator $A$ of $V$ such that $(u, v)=\langle A u, v\rangle$, for all $u, v \in V$. By hypothesis, we have

$$
\langle A \Phi(g) u, v\rangle=(\Phi(g) u, v)=\left(u, \Phi\left(g^{-1}\right) v\right)=\left\langle A u, \Phi\left(g^{-1}\right) v\right\rangle=\langle\Phi(g) A u, v\rangle .
$$

Thus $A \Phi(g)=\Phi(g) A$ for all $g \in G$. If $\lambda$ is an eigenvalue of $A$ then the corresponding eigenspace $V_{\lambda}$ is a non-zero subspace of $V$ which is stable by the set of linear transformations $\Phi(G)$. Hence, by the irreducibility assumption, it follows that $V_{\lambda}=$ $V$, which proves the proposition.

Before tackling the next proposition it is best to recall the definitions of $P(\delta)$, $E(\delta)$ and $\Phi(\delta)$ given following Proposition 6.9.

Proposition 8.4. If $U$ is a unitary representation of $G$ on a Hilbert space $E$ and $E(\delta)$ is finite dimensional and not zero, then the spherical function $\Phi_{\delta}$ is unitary. 
Proof. Let $u, v \in E(\delta)$ and $g \in G$. Since $P(\delta)$ is self adjoint we have

$$
\begin{aligned}
\left(\Phi_{\delta}(g) u, v\right) & =(P(\delta) U(g) u, v)=(U(g) u, v)=\left(u, U\left(g^{-1}\right) v\right) \\
& =\left(u, P(\delta) U\left(g^{-1}\right) v\right)=\left(u, \Phi_{\delta}\left(g^{-1}\right) v\right) .
\end{aligned}
$$

Corollary 8.5. If $G$ is a compact group then any irreducible spherical function on $G$ is unitarizable.

Proof. If $\Phi$ is an irreducible spherical function on $G$ of type $\delta$ then there exists an irreducible finite dimensional representation $(E, U)$ of $G$ such that $\Phi=P(\delta) U$ on $E(\delta)$. Since $U$ is unitarizable from Proposition 8.4, it follows that $\Phi$ is unitarizable.

This is the reason why the issue of unitarizability was not raised in [7].

Now we go on to determine which are the unitarizable irreducible spherical functions among all of those associated to the pair $(\mathrm{SL}(2, \mathbb{C}), \mathrm{SU}(2))$.

Lemma 8.6. Let $V$ be a finite dimensional complex vector space with an inner product. If $\Phi: G \longrightarrow \operatorname{End}(V)$ is an irreducible spherical function on $G=\operatorname{SL}(2, \mathbb{C})$ and

$$
(\Omega+\bar{\Omega}) \Phi=a \Phi, \quad(\Omega-\bar{\Omega}) \Phi=b \Phi
$$

then

$$
(\Omega+\bar{\Omega}) \check{\Phi}=\bar{a} \check{\Phi}, \quad(\Omega-\bar{\Omega}) \check{\Phi}=-\bar{b} \check{\Phi} .
$$

Proof. For $X, Y \in \mathfrak{g}$ and $F: G \longrightarrow \operatorname{End}(V)$ a smooth function we have $(X Y \check{F})(e)=(Y X F)^{*}(e)$. In fact

$$
\begin{aligned}
(X Y \check{F})(e) & =\left(\frac{\partial}{\partial t} \frac{\partial}{\partial s} \check{F}(\exp t X \exp s Y)\right)_{s=t=0} \\
& =\left(\frac{\partial}{\partial t} \frac{\partial}{\partial s} F(\exp (-s Y) \exp (-t X))_{s=t=0}^{*}=(Y X F)^{*}(e) .\right.
\end{aligned}
$$

From Proposition 2.1, we get

$$
\begin{aligned}
2(\Omega+\bar{\Omega}) & =H_{1}^{2}+V_{1}^{2}+V_{2}^{2}-H_{2}^{2}-W_{1}^{2}-W_{2}^{2}, \\
\Omega-\bar{\Omega} & =-i\left(H_{1} H_{2}+V_{1} W_{2}-V_{2} W_{1}\right) .
\end{aligned}
$$

Now we can compute the eigenvalue of $\Omega+\bar{\Omega}$ corresponding to $\check{\Phi}$,

$$
((\Omega+\bar{\Omega}) \check{\Phi})(e)=((\Omega+\bar{\Omega}) \Phi)^{*}(e)=\bar{a} \Phi(e)^{*}=\bar{a} I .
$$

Similarly, since $\left[H_{1}, H_{2}\right]=\left[V_{1}, W_{2}\right]=\left[W_{1}, V_{2}\right]=0$, we have

$$
((\Omega-\bar{\Omega}) \check{\Phi})(e)=-i(i(\Omega-\bar{\Omega}) \Phi)^{*}(e)=-((\Omega-\bar{\Omega}) \Phi)^{*}(e)=-\bar{b} \Phi(e)^{*}=-\bar{b} I .
$$

This completes the proof of the lemma. 
Now, we come to the main result of this section.

Theorem 8.7. An irreducible spherical function $\Phi$ on $G$ is unitarizable if and only if the eigenvalues

$$
a=((\Omega+\bar{\Omega}) \Phi)(e) \quad \text { and } \quad b=((\Omega-\bar{\Omega}) \Phi)(e)
$$

are, respectively, real and purely imaginary.

Proof. If $\Phi$ is unitarizable there exists an inner product on $V$ such that $\Phi=\check{\Phi}$. Thus from Lemma 8.6, it follows that $a=\bar{a}$ and $b=-\bar{b}$.

Conversely, let us take on $V$ an inner product such that $\pi(k)=\Phi(k)$ becomes a unitary representation of $K$. This implies that $(D \Phi)(e)=(D \check{\Phi})(e)$ for all $D \in$ $D(K)^{K}$. Moreover, from the same Lemma 8.6 and from the hypothesis we obtain that

$$
((\Omega+\bar{\Omega}) \Phi)(e)=((\Omega+\bar{\Omega}) \check{\Phi})(e) \quad \text { and } \quad((\Omega-\bar{\Omega}) \Phi)(e)=((\Omega-\bar{\Omega}) \check{\Phi})(e) .
$$

Since $D(G)^{K}=D(K)^{K} \otimes D(G)^{G}$ and $D(G)^{G}$ is generated by the algebraically independent elements $\Omega$ and $\bar{\Omega}$, from Proposition 6.10 , it follows that $\Phi=\check{\Phi}$, as we wanted to prove.

We recall that to each irreducible spherical function $\Phi$ on $G$ of type $\pi=\pi_{\ell}$ we associated a function $H$ on $G$ defined by $H(g)=\Phi(g) \Phi_{\pi}(g)^{-1}$ which can be also viewed as a function on $\mathbb{H}=G / K$ since it is right invariant under $K$. In Sec. 4, we also introduced the functions $\tilde{H}(r)=H(0, r)$ and $H(t)=\tilde{H}(\sqrt{t})$. This last one is an eigenfunction of the differential operators $D$ and $E$.

From Remark 4.9, we easily get the following relations between the eigenvalues $a$ and $b$ of $\Omega+\bar{\Omega}$ and $\Omega-\bar{\Omega}$ corresponding to $\Phi$ and the eigenvalues $\lambda$ and $\mu$ of $D$ and $E$ corresponding to $H=H(t)$ :

$$
2 \lambda=a-b, \quad \text { and } \quad \mu=b-\ell(\ell+2) .
$$

Proposition 8.8. Let $H=\left(h_{0}(t), \ldots, h_{\ell}(t)\right)$ and $K=\left(k_{0}(t), \ldots, k_{\ell}(t)\right)$ be the functions associated, respectively, to the spherical functions $\Phi$ and $\check{\Phi}$ of type $\pi=\pi_{\ell}$. If $D H=\lambda H$ and $E H=\mu H$ then

$$
D K=(\bar{\lambda}+\bar{\mu}+\ell(\ell+2)) K \quad \text { and } \quad E K=(-\bar{\mu}-2 \ell(\ell+2)) K .
$$

Moreover

$$
k_{i}(t)=t^{(\ell-2 i) / 2} \overline{h_{\ell-i}(t)} .
$$

Proof. Let $\lambda_{1}=(D K)(e)$ and $\mu_{1}=(E K)(e)$. Then from Lemma 8.6 and (8.1), we get

$$
\begin{aligned}
2 \lambda_{1} & =\bar{a}+\bar{b}=2(\bar{\lambda}+\bar{\mu}+\ell(\ell+2)), \\
\mu_{1} & =-\bar{b}-\ell(\ell+2)=-\bar{\mu}-2 \ell(\ell+2) .
\end{aligned}
$$


To prove the second assertion, for $s>0$, we let

$$
a_{s}=\left(\begin{array}{cc}
s & 0 \\
0 & s^{-1}
\end{array}\right) .
$$

Then, using the basis $\left\{v_{i}\right\}$ of $V_{\pi}$ introduced in Sec. 4, we have $\pi\left(a_{s}\right) v_{i}=s^{\ell-2 i} v_{i}$. Also we have $p\left(a_{s}\right)=\left(0, s^{-2}\right) \in \mathbb{H}$, which says that $H\left(a_{s}\right)=H\left(0, s^{-2}\right)=\tilde{H}\left(s^{-2}\right)$. This implies that

$$
\Phi\left(a_{s}\right) v_{i}=s^{\ell-2 i} \tilde{h}_{i}\left(s^{-2}\right) v_{i} .
$$

If we consider on $V_{\pi}$ an inner product such that $\pi$ becomes a unitary representation of $K$, then the basis $\left\{v_{i}\right\}$ is orthogonal. Taking into account that the adjoint of a linear transformation defined by a diagonal matrix $T$ with respect to an orthogonal basis is the linear transformation defined by the conjugate matrix $\bar{T}$, we obtain

$$
\check{\Phi}\left(a_{s}\right) v_{i}=\Phi\left(a_{s^{-1}}\right)^{*} v_{i}=s^{-(\ell-2 i)} \overline{\tilde{h}_{i}\left(s^{2}\right)} v_{i} .
$$

On the other hand changing $\Phi$ by $\check{\Phi}$ in (8.2), we get $\check{\Phi}\left(a_{s}\right) v_{i}=s^{\ell-2 i} \tilde{k}_{i}\left(s^{-2}\right) v_{i}$. Therefore $\tilde{k}_{i}\left(s^{-2}\right)=s^{-2(\ell-2 i)} \tilde{\tilde{h}}_{i}\left(s^{2}\right)$. If we put $r=s^{-2}$ and use Lemma 4.4, we have

$$
\tilde{k}_{i}(r)=r^{(\ell-2 i)} \overline{\tilde{h}_{i}\left(r^{-1}\right)}=r^{(\ell-2 i)} \overline{\tilde{h}_{\ell-i}(r)} .
$$

Now replacing $r=\sqrt{t}$, and since $k_{i}(t)=\tilde{k}_{i}(\sqrt{t})$ and $h_{\ell-i}(t)=\tilde{h}_{\ell-i}(\sqrt{t})$ we finally get $k_{i}(t)=t^{(\ell-2 i) / 2} \overline{h_{\ell-i}(t)}$, which completes the proof of the proposition.

Corollary 8.9. In terms of the parameters $\lambda$ and $\mu$ the corresponding spherical function $\Phi$ of type $\pi=\pi_{\ell}$ is unitarizable if and only if

$$
\lambda=\bar{\lambda}+\bar{\mu}+\ell(\ell+2) \quad \text { and } \quad \mu=-\bar{\mu}-2 \ell(\ell+2) .
$$

Moreover this happens precisely when

$$
h_{i}(t)=t^{(\ell-2 i) / 2} \overline{h_{\ell-i}(t)} .
$$

Proposition 8.10. If $\Phi=\Phi_{(p, k)}$ is the irreducible spherical function of type $\ell$ associated to the parameters $(p, k)$ then $\check{\Phi}=\Phi_{\left(p^{\prime}, k^{\prime}\right)}$ where $k^{\prime}=k$ and $p^{\prime}=$ $(\ell-2 k) / 2+1-\bar{p}$.

Proof. The spherical function $\Phi_{(p, k)}$ of type $\ell$ corresponds to the eigenvalues $\mu(p, k)=-4 p(\ell-2 k)-4 k(\ell-k+1)$ and $\lambda(p, k)=4 p(p-1)$, and the spherical function $\check{\Phi}_{(p, k)}$ corresponds to the eigenvalues $\mu^{\prime}=-\bar{\mu}-2 \ell(\ell+2)$ and $\lambda^{\prime}=\bar{\lambda}+\bar{\mu}+\ell(\ell+2)$, see Proposition 8.8. Therefore the only thing we need to do is to check that $\lambda^{\prime}=\lambda\left(p^{\prime}, k^{\prime}\right)$ and that $\mu^{\prime}=\mu\left(p^{\prime}, k^{\prime}\right)$. This is a straightforward computation.

Corollary 8.11. The spherical function $\Phi_{(p, k)}$ of type $\ell$ is unitarizable if and only if

(i) when $\ell \neq 2 k, \operatorname{Re}(p)=\frac{\ell-2 k}{4}+\frac{1}{2}$,

(ii) when $\ell=2 k, \operatorname{Re}(p)=\frac{1}{2}$ or $p \in \mathbb{R}$. 
Proof. From Propositions 6.9 and 8.10, it follows that $\Phi_{(p, k)}$ is unitarizable if and only if

$$
(p, k)= \begin{cases}\left(p^{\prime}, k\right), & \\ \left(1-p^{\prime}, \ell-k\right), & \\ \left(p^{\prime}, \ell+1-k-2 p^{\prime}\right) & \text { if } \ell+1-k-2 p^{\prime} \in\{0, \ldots, \ell\}, \\ \left(1-p^{\prime}, k-1+2 p^{\prime}\right) & \text { if } k-1+2 p^{\prime} \in\{0, \ldots, \ell\}\end{cases}
$$

with $p^{\prime}=(\ell-2 k) / 2+1-\bar{p}$.

Now we just need to observe that the first and the second cases in (8.3) are respectively (i) and (ii), and that the third and fourth cases occur when $\ell=2 k$ and $2 p=1$ which is included in (ii).

Remark 8.12. Recall that in terms of the parameters $v, r$ which parametrize naturally the spherical function of type $\ell$ associated to the principal series representation $U^{\sigma, \nu}$ we have

$$
v=4 i\left(\frac{\ell-2 k}{4}+\frac{1}{2}-p\right) \quad \text { and } \quad r=2 k-\ell,
$$

and thus if $\ell \neq 2 k$ the previous Corollary says that $\Phi_{v, r}$ is unitarizable exactly when $v$ is real. This corresponds, as is well known, to the cases when $U^{\sigma, \nu}$ is unitary (see [11]). If $\ell=2 k(r=0)$, we see that $\Phi_{v, 0}$ is unitarizable when $v$ is either real or purely imaginary; when $w=i v$ satisfies $0<w<2$, we are precisely in the case of the complementary series of $\operatorname{SL}(2, \mathbb{C})$.

\section{A Matrix Valued Form of the Bispectral Property}

We close this paper by displaying a rather intriguing matrix valued three term recursion relation that we have found for a function put together from our spherical functions. A similar relation was conjectured in [7] and proved in [8]. In some sense the relation in those papers is more natural than the one found here since there we were dealing with a compact situation. Here, in spite of the fact that we are in a non-compact case we obtain, once again, a three-term recursion. It may appear more natural to search for a differential equation in the spectral parameter, and we start by discussing this point first.

In the classical case, with $\ell=0$, the spherical function is given after setting $s=\log t$ by

$$
H(t, p)=H(s, p)=\frac{\sinh ((2 p-1) s / 2)}{(2 p-1) \sinh (s / 2)},
$$

and the differential equation $D H=\lambda H$ reads

$$
t^{2} H_{t t}-\frac{2 t^{2}}{1-t} H_{t}=(p-1) p H,
$$


or in the variable $s$,

$$
H_{s s}+\operatorname{coth}(s / 2) H_{s}=(p-1) p H .
$$

In this case we get a differential equation in $p$, namely

$$
H_{p p}+\frac{4}{2 p-1} H=s^{2} H .
$$

This result, and the fact that in this case we are dealing with a special case of the Jacobi functions $(\alpha=1 / 2, \beta=1 / 2)$ led one of us, see [4], to inquire if this type of "bispectral situation" could hold in a more general setup. A tentative negative answer to this question for other values of $\alpha, \beta$ is given in [12, Sec. 10]. A very detailed analysis of this issue led to [3], from which it is clear that the question in [4] has indeed a negative answer except in very exceptional cases, such as the one that leads to the three dimensional hyperbolic space. We now take up this question in the context of matrix valued spherical functions.

For a given nonnegative integer $\ell$ consider the matrix whose rows are given by the vectors $H(t)$ corresponding to the values $k=0,1,2, \ldots, \ell$ discussed in the comments preceding and following Theorem 6.8. Denote the corresponding matrix by

$$
\Phi(t, p) \text {. }
$$

In the spirit of $[7,8]$ one could look for a relation of the type

$$
A(p) \Phi_{p p}(t, p)+B(p) \Phi_{p}(t, p)+C(p) \Phi(t, p)=M(t) \Phi(p, t),
$$

where $A(p), B(p), C(p)$ are matrices independent of $t$ and the matrix $M(t)$ is independent of $p$. Of course, we could try to place the coefficient matrices to the right of the function $\Phi(t, p)$ and its derivatives, and this would lead to another set of possible differential equations in the spectral parameter.

We have succeeded in finding such matrices (by allowing for the second possibility only) but the results are rather disappointing: the matrices in question are scalar functions multiplied by the matrix made up of all ones. When one looks at this result and observes the fact that the trace of each one of our (diagonal) matrix valued functions $H(t, p)$ equals $(\ell+1)$ times the value of these functions for $\ell=0$, we see that nothing new has been learned. As we see below the situation is very different if one looks for three term recursions.

\subsection{The bispectral property}

As a function of $t, \Phi(t, p)$ satisfies two differential equations

$$
D \Phi(t, p)^{t}=\Phi(t, p)^{t} \Lambda, \quad E \Phi(t, p)^{t}=\Phi(t, p)^{t} M
$$

$D$ and $E$ are the differential operators introduced earlier. Moreover we have

Conjecture 9.1. There exist matrices $A_{p}, B_{p}, C_{p}$, independent of $t$, such that

$$
A_{p} \Phi(t, p-1)+B_{p} \Phi(t, p)+C_{p} \Phi(t, p+1)=\frac{t^{2}+1}{t} \Phi(t, p) .
$$


The matrices $A_{p}$ (upper) and $C_{p}$ (lower) consist, in general, of three diagonals each and $B_{p}$ is tridiagonal. Recall that for $t=1$, the matrix $\Phi(1, p)$ consists of all ones.

We illustrate these results below for $\ell=0,1,3,4$.

(a) $\ell=0$. Here we have

$$
\Phi(t, p)=\frac{t^{p}-t^{1-p}}{(2 p-1)(t-1)}
$$

and our property reads

$$
\frac{2 p-3}{2 p-1} \Phi(t, p-1)+\frac{2 p+1}{2 p-1} \Phi(t, p+1)=\frac{t^{2}+1}{t} \Phi(t, p) .
$$

(b) $\ell=1$. Here we have $\mu=-4 p, 4 p-4$, and for the matrix $\Phi$ we get

$$
\Phi(t, p)=\left(\begin{array}{cc}
\frac{(2 p-2) t^{2 p+1}-(2 p-1) t^{2 p}+t^{2}}{(p-1)(2 p-1)(t-1)^{2} t^{p}} & \frac{t^{2 p}+(1-2 p) t^{2}+2(p-1) t}{(p-1)(2 p-1)(t-1)^{2} t^{p}} \\
\frac{t^{1-p}\left(t^{2 p}-2 p t+2 p-1\right)}{p(2 p-1)(t-1)^{2}} & \frac{(2 p-1) t^{2 p+1}-2 p t^{2 p}+t}{p(2 p-1)(t-1)^{2} t^{p}}
\end{array}\right)
$$

and our property reads

$$
\begin{aligned}
& \left(\begin{array}{cc}
\frac{p-2}{p-1} & \frac{1}{(p-1)(2 p-1)} \\
0 & \frac{2 p-3}{2 p-1}
\end{array}\right) \Phi(t, p-1) \\
& +\left(\begin{array}{cc}
\frac{1}{(1-p)(2 p-1)} & \frac{1}{(p-1)(2 p-1)} \\
\frac{1}{p(2 p-1)} & \frac{1}{p(1-2 p)}
\end{array}\right) \Phi(t, p) \\
& +\left(\begin{array}{cc}
\frac{2 p+1}{2 p-1} & 0 \\
\frac{1}{p(2 p-1)} & \frac{p+1}{p}
\end{array}\right) \Phi(t, p+1)=\frac{t^{2}+1}{t} \Phi(t, p)
\end{aligned}
$$

(c) $\ell=3$. Here we have $\mu=-12 p,-4 p-12,4 p-16,12 p-12$, and with $\Phi(t, p)$ defined as above we get

$$
A_{p} \Phi(t, p-1)+B_{p} \Phi(t, p)+C_{p} \Phi(t, p+1)=\frac{t^{2}+1}{t} \Phi(t, p)
$$


with

$$
A_{p}=\left(\begin{array}{cccc}
\frac{p-3}{p-2} & \frac{3}{(p-1)(2 p-3)} & \frac{3}{(p-2)(p-1)(2 p-3)(2 p-1)} & 0 \\
0 & \frac{(p-2) p(2 p-5)}{(p-1)^{2}(2 p-3)} & \frac{16(p-2) p}{(p-1)(2 p-3)(2 p-1)^{2}} & \frac{3}{(p-1)^{2}(2 p-1)^{2}} \\
0 & 0 & \frac{(p-2)(2 p-3)(2 p+1)}{(p-1)(2 p-1)^{2}} & \frac{3(2 p-3)}{(p-1)(2 p-1)^{2}} \\
0 & 0 & 0 & \frac{2 p-3}{2 p-1}
\end{array}\right),
$$

$$
B_{p}=\left(\begin{array}{cccc}
-\frac{3}{(p-1)(2 p-3)} & \frac{3}{(p-1)(2 p-3)} & 0 & 0 \\
\frac{3(p-2)}{(p-1)^{2}(2 p-3)} & -\frac{14 p^{2}-21 p-9}{(p-1) p(2 p-3)(2 p-1)} & \frac{(2 p-3)(2 p+1)}{(p-1)^{2} p(2 p-1)} & 0 \\
0 & \frac{(2 p-3)(2 p+1)}{(p-1) p^{2}(2 p-1)} & -\frac{14 p^{2}-7 p-16}{(p-1) p(2 p-1)(2 p+1)} & \frac{3(p+1)}{p^{2}(2 p+1)} \\
0 & 0 & \frac{3}{p(2 p+1)} & -\frac{3}{p(2 p+1)}
\end{array}\right),
$$

$$
C_{p}=\left(\begin{array}{cccc}
\frac{2 p+1}{2 p-1} & 0 & 0 & 0 \\
\frac{3(2 p+1)}{p(2 p-1)^{2}} & \frac{(p+1)(2 p-3)(2 p+1)}{p(2 p-1)^{2}} & 0 & 0 \\
\frac{3}{p^{2}(2 p-1)^{2}} & \frac{16(p-1)(p+1)}{p(2 p-1)^{2}(2 p+1)} & \frac{(p-1)(p+1)(2 p+3)}{p^{2}(2 p+1)} & 0 \\
0 & \frac{3}{p(p+1)(2 p-1)(2 p+1)} & \frac{3}{p(2 p+1)} & \frac{p+2}{p+1}
\end{array}\right) .
$$

(d) $\ell=4$. Here we have $\mu=-16 p,-8 p-16,-24,8 p-24,16 p-16$, and with $\Phi(t, p)$ defined as above we have

$$
A_{p} \Phi(t, p-1)+B_{p} \Phi(t, p)+C_{p} \Phi(t, p+1)=\frac{t^{2}+1}{t} \Phi(t, p),
$$

where $A_{p}$ is of the form

$$
A_{p}=\left(\begin{array}{ccccc}
a_{00} & a_{01} & a_{02} & 0 & 0 \\
0 & a_{11} & a_{12} & a_{13} & 0 \\
0 & 0 & a_{22} & a_{23} & a_{24} \\
0 & 0 & 0 & a_{33} & a_{34} \\
0 & 0 & 0 & 0 & a_{44}
\end{array}\right)
$$


with

$$
\begin{array}{ll}
a_{00}=\frac{2 p-7}{2 p-5}, & a_{01}=\frac{2}{(p-2)(p-1)} \\
a_{02}=\frac{6(p-2)^{-1}(p-1)^{-1}}{(2 p-5)(2 p-1)}, & \\
a_{11}=\frac{(p-3) p(2 p-5)}{(p-2)(p-1)(2 p-3)}, & a_{12}=\frac{3 p(2 p-5)}{(p-2)(p-1)^{2}(2 p-1)} \\
a_{13}=\frac{9}{(p-1)^{2}(2 p-3)(2 p-1)}, & \\
a_{22}=\frac{p(p-2)(2 p-5)(2 p+1)}{(p-1)^{2}(2 p-1)^{2}}, & a_{23}=\frac{3(p-2)(2 p+1)}{(p-1)^{2} p(2 p-1)} \\
a_{24}= & \frac{6}{(p-1) p(2 p-1)^{2}}, \\
a_{33}= & \frac{(p-2)(p+1)(2 p-3)}{(p-1) p(2 p-1)}, \\
a_{44}= & \frac{2 p-3}{2 p-1} \cdot
\end{array}
$$

The matrix $B_{p}$ is of the form

$$
B_{p}=\left(\begin{array}{ccccc}
b_{00} & b_{01} & 0 & 0 & 0 \\
b_{10} & b_{11} & b_{12} & 0 & 0 \\
0 & b_{21} & b_{22} & b_{23} & 0 \\
0 & 0 & b_{32} & b_{33} & b_{34} \\
0 & 0 & 0 & b_{43} & b_{44}
\end{array}\right)
$$

with

$$
\begin{array}{ll}
b_{00}=-\frac{2}{(p-2)(p-1)}, & b_{01}=\frac{2}{(p-2)(p-1)} \\
b_{10}=\frac{2(2 p-5)}{(p-2)(p-1)(2 p-3)}, & b_{11}=-\frac{5 p^{2}-10 p-4}{(p-2)(p-1)^{2} p} \\
b_{12}=\frac{3(p-2)(2 p+1)}{(p-1)^{2} p(2 p-3)}, & \\
b_{21}=\frac{3(p-2)(2 p+1)}{(p-1)^{2} p(2 p-1)}, & b_{22}=-\frac{3\left(2 p^{2}-2 p-3\right)}{(p-1)^{2} p^{2}} \\
b_{23}=\frac{3(p+1)(2 p-3)}{(p-1) p^{2}(2 p-1)}, &
\end{array}
$$




$$
\begin{array}{ll}
b_{32}=\frac{3(p+1)(2 p-3)}{(p-1) p^{2}(2 p+1)}, & b_{33}=-\frac{5 p^{2}-9}{(p-1) p^{2}(p+1)}, \\
b_{34}=\frac{2(2 p+3)}{p(p+1)(2 p+1)}, & \\
b_{43}=\frac{2}{p(p+1)}, & b_{44}=-\frac{2}{p(p+1)} .
\end{array}
$$

The matrix $C_{p}$ is of the form

$$
C_{p}=\left(\begin{array}{ccccc}
c_{00} & 0 & 0 & 0 & 0 \\
c_{10} & c_{11} & 0 & 0 & 0 \\
c_{20} & c_{21} & c_{22} & 0 & 0 \\
0 & c_{31} & c_{32} & c_{33} & 0 \\
0 & 0 & c_{42} & c_{43} & c_{44}
\end{array}\right)
$$

with

$$
\begin{aligned}
& c_{00}=\frac{2 p+1}{2 p-1}, \\
& c_{10}=\frac{2(2 p+1)}{(p-1) p(2 p-1)}, \\
& c_{11}=\frac{(p-2)(p+1)(2 p+1)}{(p-1) p(2 p-1)}, \\
& c_{20}=\frac{6}{(p-1) p(2 p-1)^{2}}, \\
& c_{21}=\frac{3(p+1)(2 p-3)}{(p-1) p^{2}(2 p-1)}, \\
& c_{22}=\frac{(p-1)(p+1)(2 p-3)(2 p+3)}{p^{2}(2 p-1)^{2}}, \\
& c_{31}=\frac{9}{p^{2}(2 p-1)(2 p+1)}, \\
& c_{32}=\frac{3(p-1)(2 p+3)}{p^{2}(p+1)(2 p-1)}, \\
& c_{33}=\frac{(p-1)(p+2)(2 p+3)}{p(p+1)(2 p+1)}, \\
& c_{42}=\frac{6(p+1)^{-1}}{p(2 p-1)(2 p+3)}, \\
& c_{43}=\frac{2}{p(p+1)}, \\
& c_{44}=\frac{2 p+5}{2 p+3} \text {. }
\end{aligned}
$$

\section{Acknowledgments}

This paper is partially supported by NSF grants FD9971151 and 1-443964-21160 and by CONICET grant PIP655-98. 


\section{Appendix A}

For completeness we include here the proof of those propositions and lemmas stated in the paper in Secs. 3 and 4. We start with the first order differential operator $E$.

Proof of Proposition 3.4. By (3.2), we have

$$
\begin{aligned}
E H= & H_{1}(H) \Phi_{\pi} \dot{\pi}\left(H_{1}\right) \Phi_{\pi}^{-1}+V_{1}(H) \Phi_{\pi} \dot{\pi}\left(V_{1}\right) \Phi_{\pi}^{-1}+V_{2}(H) \Phi_{\pi} \dot{\pi}\left(V_{2}\right) \Phi_{\pi}^{-1} \\
= & H_{1}(H) \Phi_{\pi} \dot{\pi}\left(H_{1}\right) \Phi_{\pi}^{-1}+\left(V_{1}(H)+i V_{2}(H)\right) \Phi_{\pi} \dot{\pi}\left(E_{12}\right) \Phi_{\pi}^{-1} \\
& +\left(V_{1}(H)-i V_{2}(H)\right) \Phi_{\pi} \dot{\pi}\left(E_{21}\right) \Phi_{\pi}^{-1}
\end{aligned}
$$

We start by computing $H_{1}(H)$. For $g=\left(\begin{array}{ll}a & b \\ c & d\end{array}\right) \in G$, we have

$$
\begin{aligned}
p\left(g \exp t H_{1}\right) & =p\left(g\left(\begin{array}{cc}
e^{t} & 0 \\
0 & e^{-t}
\end{array}\right)\right)=g\left(\begin{array}{cc}
e^{2 t} & 0 \\
0 & e^{-2 t}
\end{array}\right) g^{*}\left(\begin{array}{l}
0 \\
1
\end{array}\right) \\
& =\left(\begin{array}{c}
a \bar{c} e^{2 t}+b \bar{d} e^{-2 t} \\
|c|^{2} e^{2 t}+|d|^{2} e^{-2 t}
\end{array}\right) \\
& =\left(\begin{array}{c}
u(t) \\
v(t)
\end{array}\right)=\left(\begin{array}{c}
u_{1}(t)+i u_{2}(t) \\
v(t)
\end{array}\right) .
\end{aligned}
$$

Also we have

$$
\begin{aligned}
& \left(\frac{d u}{d t}\right)_{t=0}=\left(\frac{d u_{1}}{d t}\right)_{t=0}+i\left(\frac{d u_{2}}{d t}\right)_{t=0}=2(a \bar{c}-b \bar{d}), \\
& \left(\frac{d v}{d t}\right)_{t=0}=2\left(|c|^{2}-|d|^{2}\right) .
\end{aligned}
$$

By the chain rule, we get

$$
H_{1}(H)(g)=2 H_{x} \operatorname{Re}(a \bar{c}-b \bar{d})+2 H_{y} \operatorname{Im}(a \bar{c}-b \bar{d})+2 H_{r}\left(|c|^{2}-|d|^{2}\right) .
$$

For $V_{1}$, we have

$$
\begin{aligned}
p\left(g \exp t V_{1}\right) & =g \exp t V_{1}\left(\exp t V_{1}\right)^{*} g^{*}\left(\begin{array}{l}
0 \\
1
\end{array}\right) \\
& =\left(\begin{array}{ll}
a & b \\
c & d
\end{array}\right)\left(\begin{array}{c}
\cosh t \sinh t \\
\sinh t \cosh t
\end{array}\right)\left(\begin{array}{c}
\cosh t \sinh t \\
\sinh t \cosh t
\end{array}\right)\left(\begin{array}{ll}
\bar{a} & \bar{c} \\
\bar{b} & \bar{d}
\end{array}\right)\left(\begin{array}{l}
0 \\
1
\end{array}\right) \\
& =\left(\begin{array}{c}
\left(\cosh ^{2} t+\sinh ^{2} t\right)(a \bar{c}+b \bar{d})+2(b \bar{c}+a \bar{d}) \sinh t \cosh t \\
\left(\cosh ^{2} t+\sinh ^{2} t\right)\left(|c|^{2}+|d|^{2}\right)+2(d \bar{c}+c \bar{d}) \sinh t \cosh t
\end{array}\right) \\
& =\left(\begin{array}{c}
\tilde{u}(t) \\
\tilde{v}(t)
\end{array}\right)=\left(\begin{array}{c}
\tilde{u}_{1}(t)+i \tilde{u}_{2}(t) \\
\tilde{v}(t)
\end{array}\right) .
\end{aligned}
$$


Thus,

$$
\left(\frac{d \tilde{u}}{d t}\right)_{t=0}=2(b \bar{c}+a \bar{d}), \quad\left(\frac{d \tilde{v}}{d t}\right)_{t=0}=2(d \bar{c}+c \bar{d}) .
$$

We get

$$
V_{1}(H)(g)=2 H_{x} \operatorname{Re}(b \bar{c}+a \bar{d})+2 H_{y} \operatorname{Im}(b \bar{c}+a \bar{d})+2 H_{r}(d \bar{c}+c \bar{d}) .
$$

Similarly, we get

$$
V_{2}(H)(g)=2 H_{x} \operatorname{Im}(-b \bar{c}+a \bar{d})-2 H_{y} \operatorname{Re}(b \bar{c}-a \bar{d})+2 i H_{r}(c \bar{d}-d \bar{c}) .
$$

Therefore

$$
\begin{aligned}
& \left(V_{1}(H)+i V_{2}(H)\right)(g)=2 H_{x}(b \bar{c}+\bar{a} d)-2 i H_{y}(b \bar{c}-\bar{a} d)+4 H_{r} d \bar{c}, \\
& \left(V_{1}(H)-i V_{2}(H)\right)(g)=2 H_{x}(\bar{b} c+a \bar{d})+2 i H_{y}(\bar{b} c-a \bar{d})+4 H_{r} c \bar{d} .
\end{aligned}
$$

On the other hand, we compute

$$
\Phi_{\pi}(g) \dot{\pi}(X) \Phi_{\pi}^{-1}(g)=\left(\frac{d}{d t}\right)_{t=0} \pi\left(g \exp t X g^{-1}\right)=\dot{\pi}\left(g X g^{-1}\right) .
$$

Then

$$
\begin{aligned}
& \Phi_{\pi}(g) \dot{\pi}\left(E_{12}\right) \Phi_{\pi}^{-1}(g)=\dot{\pi}\left(\begin{array}{cc}
-a c & a^{2} \\
-c^{2} & a c
\end{array}\right), \\
& \Phi_{\pi}(g) \dot{\pi}\left(E_{21}\right) \Phi_{\pi}^{-1}(g)=\dot{\pi}\left(\begin{array}{cc}
b d & -b^{2} \\
d^{2} & -b d
\end{array}\right), \\
& \Phi_{\pi}(g) \dot{\pi}\left(H_{1}\right) \Phi_{\pi}^{-1}(g)=\dot{\pi}\left(\begin{array}{cc}
a d+b c & -2 a b \\
2 c d & -a d-b c
\end{array}\right) .
\end{aligned}
$$

Therefore, by (A.1), we have $E H(g)=H_{x} A+H_{y} B+H_{r} C$, where

$$
\begin{aligned}
A= & 2(b \bar{c}+\bar{a} d) \dot{\pi}\left(\begin{array}{cc}
-a c & a^{2} \\
-c^{2} & a c
\end{array}\right)+2(\bar{b} c+a \bar{d}) \dot{\pi}\left(\begin{array}{cc}
b d & -b^{2} \\
d^{2} & -b d
\end{array}\right) \\
& +2 \operatorname{Re}(a \bar{c}-b \bar{d}) \dot{\pi}\left(\begin{array}{cc}
a d+b c & -2 a b \\
2 c d & -a d-b c
\end{array}\right), \\
B= & -2 i(b \bar{c}-\bar{a} d) \dot{\pi}\left(\begin{array}{cc}
-a c & a^{2} \\
-c^{2} & a c
\end{array}\right)+2 i(\bar{b} c-a \bar{d}) \dot{\pi}\left(\begin{array}{cc}
b d & -b^{2} \\
d^{2} & -b d
\end{array}\right) \\
& +2 \operatorname{Im}(a \bar{c}-b \bar{d}) \dot{\pi}\left(\begin{array}{cc}
a d+b c & -2 a b \\
2 c d & -a d-b c
\end{array}\right),
\end{aligned}
$$




$$
\begin{aligned}
C= & 4 d \bar{c} \dot{\pi}\left(\begin{array}{cc}
-a c & a^{2} \\
-c^{2} & a c
\end{array}\right)+4 \bar{d} c \dot{\pi}\left(\begin{array}{cc}
b d & -b^{2} \\
d^{2} & -b d
\end{array}\right) \\
& +2\left(|c|^{2}-|d|^{2}\right) \dot{\pi}\left(\begin{array}{cc}
a d+b c & -2 a b \\
2 c d & -a d-b c
\end{array}\right) .
\end{aligned}
$$

By means of straightforward calculations we obtain

$$
\begin{aligned}
& A=\dot{\pi}\left(\begin{array}{cc}
z-\bar{z} & \frac{2\left(1+|z|^{2}\right)}{r} \\
2 r & \bar{z}-z
\end{array}\right), \quad B=\dot{\pi}\left(\begin{array}{cc}
-i(z+\bar{z}) & \frac{2 i\left(1+|z|^{2}\right)}{r} \\
-2 i r & i(z+\bar{z})
\end{array}\right), \\
& C=\dot{\pi}\left(\begin{array}{cc}
-2 r & 4 z \\
0 & 2 r
\end{array}\right) .
\end{aligned}
$$

This completes the proof of Proposition 3.4.

Proof of Proposition 3.3. We have $D H=\left(H_{1}^{2}+V_{1}^{2}+V_{2}^{2}\right) H$. For any $X \in \mathfrak{g}$ one has

$$
X^{2}(H)(g)=\left(\frac{d}{d s} \frac{d}{d t} H(p(g \exp (s+t) X))\right)_{s=t=0} .
$$

Let us introduce the functions $u, v, u_{1}, u_{2}$ by means of

$$
p(g \exp (s+t) X)=(u(s, t), v(s, t))=\left(u_{1}(s, t), u_{2}(s, t), v(s, t)\right),
$$

where $u(s, t)=u_{1}(s, t)+i u_{2}(s, t)$ and $u_{1}, u_{2}, v$ are real valued functions. We note that if $u(t)=u_{1}(t)+i u_{2}(t)$, then $\frac{d u_{1}}{d t}=\operatorname{Re}\left(\frac{d u}{d t}\right)$ and $\frac{d u_{2}}{d t}=\operatorname{Im}\left(\frac{d u}{d t}\right)$. Using the chain rule we have

$$
\begin{aligned}
X^{2}(H)(g)= & H_{x x} \frac{\partial u_{1}}{\partial s} \frac{\partial u_{1}}{\partial t}+H_{y y} \frac{\partial u_{2}}{\partial s} \frac{\partial u_{2}}{\partial t}+H_{r r} \frac{\partial v}{\partial s} \frac{\partial v}{\partial t} \\
& +H_{x y}\left(\frac{\partial u_{1}}{\partial s} \frac{\partial u_{2}}{\partial t}+\frac{\partial u_{2}}{\partial s} \frac{\partial u_{1}}{\partial t}\right)+H_{x r}\left(\frac{\partial v}{\partial s} \frac{\partial u_{1}}{\partial t}+\frac{\partial u_{1}}{\partial s} \frac{\partial v}{\partial t}\right) \\
& +H_{y r}\left(\frac{\partial v}{\partial s} \frac{\partial u_{2}}{\partial t}+\frac{\partial u_{2}}{\partial s} \frac{\partial v}{\partial t}\right)+H_{x} \frac{\partial^{2} u_{1}}{\partial t \partial s}+H_{y} \frac{\partial^{2} u_{2}}{\partial t \partial s}+H_{r} \frac{\partial^{2} v}{\partial t \partial s}
\end{aligned}
$$

We need to handle separately the cases $X=H_{1}, X=V_{1}$ and $X=V_{2}$. For $X=H_{1}$, we start with

$$
\begin{aligned}
p\left(g \exp (s+t) H_{1}\right) & =\left(\begin{array}{ll}
a & b \\
c & d
\end{array}\right)\left(\begin{array}{cc}
e^{2(t+s)} & 0 \\
0 & e^{-2(t+s)}
\end{array}\right)\left(\begin{array}{cc}
\bar{a} & \bar{c} \\
\bar{b} & \bar{d}
\end{array}\right)\left(\begin{array}{l}
0 \\
1
\end{array}\right) \\
& =\left(\begin{array}{c}
a \bar{c} e^{2(t+s)}+b \bar{d} e^{-2(t+s)} \\
|c|^{2} e^{2(t+s)}+|d|^{2} e^{-2(t+s)}
\end{array}\right) .
\end{aligned}
$$


Therefore at $s=t=0$, we have

$$
\frac{\partial u}{\partial s}=\frac{\partial u}{\partial t}=2 a \bar{c}-2 b \bar{d}, \quad \frac{\partial v}{\partial s}=\frac{\partial v}{\partial t}=2\left(|c|^{2}-|d|^{2}\right),
$$

and

$$
\frac{\partial^{2} u}{\partial s \partial t}=4(a \bar{c}+b \bar{d}), \quad \frac{\partial^{2} v}{\partial s \partial t}=4\left(|c|^{2}+|d|^{2}\right) .
$$

Thus we obtain

$$
\begin{aligned}
H_{1}^{2}(H)= & 4 H_{x x} \operatorname{Re}^{2}(a \bar{c}-b \bar{d})+4 H_{y y} \operatorname{Im}^{2}(a \bar{c}-b \bar{d})+4 H_{r r}\left(|c|^{2}-|d|^{2}\right)^{2} \\
& +8 H_{x y} \operatorname{Re}(a \bar{c}-b \bar{d}) \operatorname{Im}(a \bar{c}-b \bar{d})+8 H_{x r} \operatorname{Re}(a \bar{c}-b \bar{d})\left(|c|^{2}-|d|^{2}\right) \\
& +8 H_{y r} \operatorname{Im}(a \bar{c}-b \bar{d})\left(|c|^{2}-|d|^{2}\right)+4 H_{x} \operatorname{Re}(a \bar{c}+b \bar{d}) \\
& +4 H_{y} \operatorname{Im}(a \bar{c}+b \bar{d})+4 H_{r}\left(|c|^{2}+|d|^{2}\right) .
\end{aligned}
$$

For $X=V_{1}$, we have $\exp t V_{1}=\left(\begin{array}{cc}\cosh t & \sinh t \\ -\sinh t & \cosh t\end{array}\right)$ and $p\left(g \exp (s+t) V_{1}\right)=$ $(u(s, t), v(s, t))$ where

$$
\begin{aligned}
u(s, t)= & \left(\cosh ^{2}(t+s)+\sinh ^{2}(t+s)\right)(a \bar{c}+b \bar{d}) \\
& +2 \sinh (s+t) \cosh (s+t)(b \bar{c}+a \bar{d}), \\
v(s, t)= & \left(\cosh ^{2}(t+s)+\sinh ^{2}(t+s)\right)\left(|c|^{2}+|d|^{2}\right) \\
& +2 \sinh (s+t) \cosh (s+t)(d \bar{c}+c \bar{d}) .
\end{aligned}
$$

Therefore at $s=t=0$, we have

$$
\begin{aligned}
\frac{\partial u}{\partial s} & =\frac{\partial u}{\partial t}=2(b \bar{c}+a \bar{d}), & \frac{\partial v}{\partial s} & =\frac{\partial v}{\partial t}=2(d \bar{c}+c \bar{d}), \\
\frac{\partial^{2} u}{\partial s \partial t} & =4(a \bar{c}+b \bar{d}), & \frac{\partial^{2} v}{\partial s \partial t} & =4\left(|c|^{2}+|d|^{2}\right),
\end{aligned}
$$

and we obtain

$$
\begin{aligned}
V_{1}^{2}(H)= & 4 H_{x x} \operatorname{Re}^{2}(b \bar{c}+a \bar{d})+4 H_{y y} \operatorname{Im}^{2}(b \bar{c}+a \bar{d})+4 H_{r r}(d \bar{c}+c \bar{d})^{2} \\
& +8 H_{x y} \operatorname{Re}(b \bar{c}+a \bar{d}) \operatorname{Im}(b \bar{c}+a \bar{d})+8 H_{x r}(d \bar{c}+c \bar{d}) \operatorname{Re}(b \bar{c}+a \bar{d}) \\
& +8 H_{y r}(d \bar{c}+c \bar{d}) \operatorname{Im}(b \bar{c}+a \bar{d})+4 H_{x} \operatorname{Re}(a \bar{c}+b \bar{d}) \\
& +4 H_{y} \operatorname{Im}(a \bar{c}+b \bar{d})+4 H_{r}\left(|c|^{2}+|d|^{2}\right) .
\end{aligned}
$$

Finally for $X=V_{2}$, we obtain

$$
\begin{aligned}
V_{2}^{2}(H)= & 4 H_{x x} \operatorname{Im}^{2}(a \bar{d}-b \bar{c})+4 H_{y y} \operatorname{re}^{2}(a \bar{d}-b \bar{c})-4 H_{r r}(c \bar{d}-d \bar{c})^{2} \\
& -8 H_{x y} \operatorname{Re}(a \bar{d}-b \bar{c}) \operatorname{Im}(a \bar{d}-b \bar{c})-8 i H_{x r}(c \bar{d}-d \bar{c}) \operatorname{Im}(a \bar{d}-b \bar{c}) \\
& +8 i H_{y r}(c \bar{d}-d \bar{c}) \operatorname{Re}(a \bar{d}-b \bar{c})+4 H_{x} \operatorname{Re}(a \bar{c}+b \bar{d}) \\
& +4 H_{y} \operatorname{Im}(a \bar{c}+b \bar{d})+4 H_{r}\left(|c|^{2}+|d|^{2}\right) .
\end{aligned}
$$


Now, the proposition follows from Eqs. (A.2), (A.3) and (A.4) and by making use of the expressions

$$
r=|c|^{2}+|d|^{2} \quad \text { and } \quad z=a \bar{c}+b \bar{d}
$$

Now we shall compute all first and second order partial derivatives that we used in Sec. 4. Given $(z, r) \in \mathbb{H}, z=z_{1}+i z_{2}, z_{1}, z_{2} \in \mathbb{R}, r \in \mathbb{R}_{>0}$, we define

$$
\begin{aligned}
& s=\frac{1}{2 r}\left(1+|z|^{2}+r^{2}-\sqrt{\left(|z|^{2}+(r+1)^{2}\right)\left(|z|^{2}+(1-r)^{2}\right)}\right), \\
& a=\frac{1-r s}{\sqrt{(1-r s)^{2}+(s|z|)^{2}}} \quad \text { and } \quad b=\frac{s z}{\sqrt{(1-r s)^{2}+(s|z|)^{2}}} .
\end{aligned}
$$

Then the matrix $A=A(z, r)=\left(\begin{array}{cc}\bar{a} & -b \\ \bar{b} & a\end{array}\right)$ satisfies $(z, r)=A \cdot(0, s)$. In fact in Sec. 4, we defined a function $k=k(z, r)$ such that $k(z, r) \cdot(z, r)=(0, s)$, and $A=k^{-1}$. Therefore

$$
H(z, r)=\pi(A(z, r)) \tilde{H}(s(z, r)) \pi\left(A(z, r)^{-1}\right) .
$$

Lemma A.1. At $(0, r) \in \mathbb{H}$, we have

$$
\frac{\partial H}{\partial z_{j}}(0, r)=\left[\dot{\pi}\left(\frac{\partial A}{\partial z_{j}}\right), H(r)\right]
$$

and

$$
\begin{aligned}
\frac{\partial^{2} H}{\partial z_{j}^{2}}(0, r)= & -\frac{2 r}{1-r^{2}} \frac{d \tilde{H}}{d r}+\frac{\partial^{2}(\pi \circ A)}{\partial z_{j}^{2}} \tilde{H}(r)+\tilde{H}(r) \frac{\partial^{2}\left(\pi \circ A^{-1}\right)}{\partial z_{j}^{2}} \\
& -2 \dot{\pi}\left(\frac{\partial A}{\partial z_{j}}\right) \tilde{H}(r) \dot{\pi}\left(\frac{\partial A}{\partial z_{j}}\right) .
\end{aligned}
$$

Proof. We have

$$
H(z, r)=\pi(A(z, r)) \tilde{H}(s(z, r)) \pi\left(A(z, r)^{-1}\right) .
$$

Then for $j=1,2$

$$
\begin{aligned}
\frac{\partial H}{\partial z_{j}}= & \frac{\partial(\pi \circ A)}{\partial z_{j}}(\tilde{H} \circ s)\left(\pi \circ A^{-1}\right)+(\pi \circ A) \frac{\partial(\tilde{H} \circ s)}{\partial z_{j}}\left(\pi \circ A^{-1}\right) \\
& +(\pi \circ A)(\tilde{H} \circ s) \frac{\partial\left(\pi \circ A^{-1}\right)}{\partial z_{j}} .
\end{aligned}
$$

We also have

$$
\frac{\partial(\tilde{H} \circ s)}{\partial z_{j}}=\left(\frac{d \tilde{H}}{d r} \circ s\right) \frac{\partial s}{\partial z_{j}} .
$$

Note that $s(0, r)=r, A(r, 0)=I$ and that at $(r, 0)$

$$
\frac{\partial s}{\partial z_{j}}=0, \quad \frac{\partial(\pi \circ A)}{\partial z_{j}}=\dot{\pi}\left(\frac{\partial A}{\partial z_{j}}\right), \quad \frac{\partial\left(\pi \circ A^{-1}\right)}{\partial z_{j}}=-\dot{\pi}\left(\frac{\partial A}{\partial z_{j}}\right) .
$$


Therefore $\frac{\partial H}{\partial z_{j}}(0, r)=\left[\dot{\pi}\left(\frac{\partial A}{\partial z_{j}}\right), H(r)\right]$.

To compute the second order derivatives we start by observing that at $(r, 0)$, we have

$$
\frac{\partial^{2}(\tilde{H} \circ s)}{\partial z_{j}^{2}}=\frac{d^{2} \tilde{H}}{d r^{2}}\left(\frac{\partial s}{\partial z_{j}}\right)^{2}+\frac{d \tilde{H}}{d r} \frac{\partial^{2} s}{\partial z_{j}^{2}}=-\frac{2 r}{1-r^{2}} \frac{d \tilde{H}}{d r} .
$$

Now the lemma follows by differentiating the expression given in (A.7) and evaluating it at $(r, 0) \in \mathbb{C}^{2}$.

Lemma A.2. At $(0, r) \in \mathbb{H}$, we have

$$
\frac{\partial^{2}(\pi \circ A)}{\partial z_{j}^{2}}=\dot{\pi}\left(\frac{\partial^{2} A}{\partial z_{j}^{2}}\right)-\dot{\pi}\left(\left(\frac{\partial A}{\partial z_{j}}\right)^{2}\right)+\dot{\pi}\left(\frac{\partial A}{\partial z_{j}}\right)^{2},
$$

and

$$
\frac{\partial^{2}\left(\pi \circ A^{-1}\right)}{\partial z_{j}^{2}}=-\dot{\pi}\left(\frac{\partial^{2} A}{\partial z_{j}^{2}}\right)+\dot{\pi}\left(\left(\frac{\partial A}{\partial z_{j}}\right)^{2}\right)+\dot{\pi}\left(\frac{\partial A}{\partial z_{j}}\right)^{2} .
$$

For the proof, see [7, Proposition 13.3]. Here $\pi$ refers to its extension to $\mathrm{GL}(2, \mathbb{C})$ which is trivial on the center.

Finally we give the proof of the following proposition, stated in Sec. 4.

Proof of Proposition 4.1. The matrix $A$ in the previous lemmas is defined by $A=A(z, r)=\left(\begin{array}{cc}\bar{a} & -b \\ \bar{b} & a\end{array}\right)$ with $a, b$ given in (A.6).

We start by computing $\frac{\partial A}{\partial z_{j}}$ and $\frac{\partial^{2} A}{\partial z_{j}^{2}}$. It is easy to verify that at $(0, r) \in \mathbb{H}$ we have

$$
\frac{\partial a}{\partial z_{j}}=0, \quad \frac{\partial^{2} a}{\partial z_{j}^{2}}=-\frac{r^{2}}{\left(1-r^{2}\right)^{2}}, \quad \frac{\partial b}{\partial z_{j}}=\delta_{j} \frac{r}{1-r^{2}}, \quad \frac{\partial^{2} b}{\partial z_{j}^{2}}=0,
$$

where $\delta_{j}=1$ if $j=1$, and $\delta_{j}=i$ if $j=2$. Then at $(0, r) \in \mathbb{H}$

$$
\frac{\partial A}{\partial z_{1}}=-\frac{r}{1-r^{2}} J, \quad \frac{\partial A}{\partial z_{2}}=-\frac{i r}{1-r^{2}} T, \quad \frac{\partial^{2} A}{\partial z_{j}^{2}}=-\frac{r^{2}}{\left(1-r^{2}\right)^{2}} I .
$$

We observe that $\left(\frac{\partial A}{\partial z_{j}}\right)^{2}=\frac{\partial^{2} A}{\partial z_{j}^{2}}$, then by Lemma A.2, we have at $(0, r)$

$$
\frac{\partial^{2}(\pi \circ A)}{\partial z_{j}^{2}}=\frac{\partial^{2}\left(\pi \circ A^{-1}\right)}{\partial z_{j}^{2}}=\dot{\pi}\left(\frac{\partial A}{\partial z_{j}}\right)^{2} .
$$

Now the proposition follows directly from Lemma A.1.

\section{References}

1. G. Andrews, R. Askey and R. Roy, Special Functions, Ency. Math. Appl. Cambridge University Press, 1999.

2. A. Erdelyi, Higher Trascendental Functions, Vol. I, ed. A. Erdelyi, McGraw-Hill. 
3. J. J. Duistermaat and F. A. Grünbaum, Differential equations in the spectral parameters, Comm. Math. Phys. 103 (1986), 177-240.

4. F. A. Grünbaum, The limited angle problem in tomography and some related mathematical problems, Proc. Intern. Colloq. Luminy, France, May 1982, North Holland, Amsterdam.

5. R. Godement, A theory of spherical functions I, Trans. Amer. Math. Soc. 73 (1952), 494-556.

6. R. Gangolli and V. S. Varadarajan, Harmonic analysis of spherical functions on real reductive groups, Series title: Ergebnisse der Mathematik und ihrer Grenzgebiete, Springer-Verlag, Berlin, New York 101, 1988.

7. F. A. Grünbaum, I. Pacharoni and J. A. Tirao, Matrix valued spherical functions associated to the complex projective plane, J. Funct. Anal. 188 (2002), 350-441.

8. F. A. Grünbaum, I. Pacharoni and J. Tirao, A matrix valued solution to Bochner's problem, J. Phys. A: Math. Gen. 34(48) (2001), 10647-10656.

9. J. Humphreys, Introduction to Lie Algebras and Representation Theory, Springer Verlag, New York, 1972.

10. S. Karlin and J. McGregor, The Hahn polynomials, formulas and an application, Scripta Math. 26 (1961), 33-46.

11. A. Knapp, Representation Theory of Semisimple Groups, Princeton University Press, Princeton, New Jersey, 1986.

12. T. Koornwinder, Jacobi functions and analysis on noncampact semisimple Lie groups, in Special Functions: Group Theoretical Aspects and Applications, ed. D. Reidel, Dordrecht, Boston, Lancaster, 1984. Series title: Mathematics and its Applications.

13. J. Tirao, Spherical functions, Rev. de la Unión Matem., Argentina 28 (1977), 75-98.

14. A. Wang, An analogue of the Payley-Wiener theorem for certain function spaces on $\mathrm{SL}(2, \mathbb{C})$, Pacific J. Math. 52(2) (1974), 617-629. 